\title{
MEASUREMENT OF POLYMER CONCENTRATION AND \\ OPTIMIZATION OF SLUDGE DEWATERING USING UV-VIS \\ SPECTROSCOPY
}

\author{
A thesis submitted to \\ the Faculty of Graduate and Postdoctoral Affairs \\ in partial fulfillment of the requirements for the degree \\ Master of Applied Science
}

by

Saman Aghamir-Baha

B.A.Sc in Chemical Engineering
Department of Civil and Environmental Engineering
Carleton University

Ottawa-Carleton Institute of Civil and Environmental Engineering

May 2014

(C) 2014

Saman Aghamir-Baha 


\begin{abstract}
Optimization of conditioning and dewatering processes is a continuing challenge for wastewater treatment plants. This study investigated the use of UV-Vis spectroscopy to measure and optimize the polymer dose during water and wastewater treatment. Residual polymer concentrations were also measured using two other methods (NBromination and Dye Method) and results were compared with the UV-Vis method. UVVis method was found to be more sensitive than the other methods in environmental samples. In addition, the study established a relationship between the optimum polymer dose and residual polymer concentration in filtrate using UV-Vis absorbance measurements at $190 \mathrm{~nm}$. Results showed that the UV-Vis method could be a potential in-line tool for real-time optimization of polymer dose during sludge dewatering and other processes that rely on coagulation and flocculation of solids.
\end{abstract}




\section{Acknowledgements}

I wish to express my gratitude to my supervisor Dr. Banu Ormeci for her endless academic and moral support. Appreciations are extended to the Environmental Engineering Laboratory supervisor Dr. Marie Chow for her never-ending assistance during the experimental research. I am grateful to my graduate student peers specially Dr. Fares Al Momani for inputting me with his knowledge.

I am indebted to my precious family as well as my boyfriend for their life-long support, including their continuous moral support during my research.

The last but not the least, I would like to acknowledge the time and effort my examination committee has put on reviewing my thesis and participating in my defense.

Once again, I am grateful to my supervisor, examiners, colleagues, my family and friends, without whose continuous support the completion of this thesis would have been much more difficult. 


\begin{tabular}{|c|c|}
\hline Centrate & Centrate of Anaerobically Digested Sludge \\
\hline CSC & Cake Solids Concentration \\
\hline CST & Capillary Suction Time \\
\hline DAF & Dissolved Air Floatation \\
\hline DG & Direct Light Resistance Black G \\
\hline DOM & Dissolved Organic Matter \\
\hline DW & Deionized Water \\
\hline FV & Filtrate Volume \\
\hline HRT & Hydraulic Retention Time \\
\hline $\mathrm{I}$ & Number of standards \\
\hline $\mathrm{J}$ & Number of replicates of each standard \\
\hline K & Number of replicates for each unknown standard \\
\hline $\mathrm{L}$ & $\begin{array}{l}\text { Number of instrument measurements corresponding to each replicate } \\
\text { of each standard }\end{array}$ \\
\hline MDL & Method Detection Limit \\
\hline PS & Primary Sludge \\
\hline ROPEC & Robert O Pickard Environmental Centre \\
\hline $\mathrm{SCD}$ & Streaming Current Detector \\
\hline Sludge & Anaerobically Digested Sludge \\
\hline SRF & Specific Resistance to Filtrations \\
\hline $\mathrm{t}_{(0.05, \mathrm{n}-2)}$ & $\begin{array}{l}\text { Students t-distribution at } 95 \% \text { confidence interval and } \mathrm{n} \text { degrees of } \\
\text { freedom }\end{array}$ \\
\hline UV-Vis & Ultraviolet Visible \\
\hline WAS & Waste Activated Sludge \\
\hline $\mathrm{X}$ & Concentration of the prepared standards \\
\hline $\bar{X}$ & $\begin{array}{l}\text { Average concentration of I number of standards with } \mathrm{J} \text { number of } \\
\text { replicates and each replicate measured L times }\end{array}$ \\
\hline $\mathrm{X}_{\mathrm{i}}$ & Concentration of each standard \\
\hline$X_{c}$ & $\begin{array}{l}\text { Critical concentration that is the closest concentration to blank that } \\
\text { can be detected via the instrument }\end{array}$ \\
\hline Y & Absorbance of the corresponding standard \\
\hline $\bar{Y}$ & $\begin{array}{l}\text { Average absorbance corresponding to I number of standards with } \mathbf{J} \\
\text { number of replicates and each replicate measured L times }\end{array}$ \\
\hline $\mathrm{Y}_{\mathrm{i}}$ & Absorbance corresponding to each standard \\
\hline$Y_{c}$ & Absorbance corresponding to critical concentration \\
\hline$\widehat{y_{l}}$ & Calculated value of calibration standard response \\
\hline $\bar{y}_{i j}$ & Average response of different replicates of the same standard \\
\hline$\lambda$ & Wavelength \\
\hline
\end{tabular}




\section{Table of Contents}

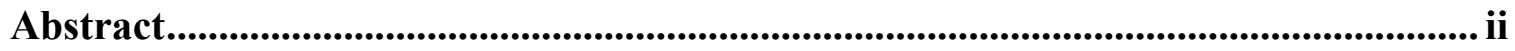

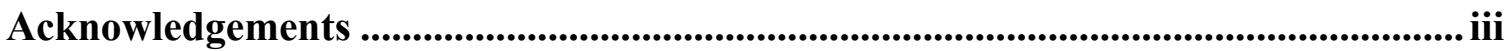

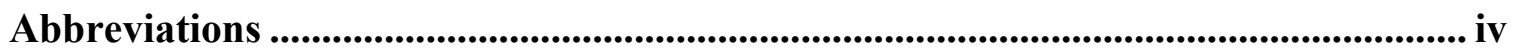

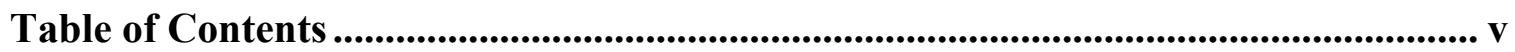

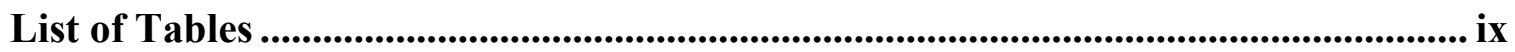

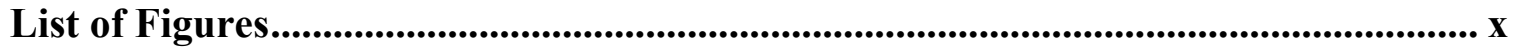

List of Appendices ....................................................................................................................... xvii

Chapter 1: Introduction ................................................................................................ 1

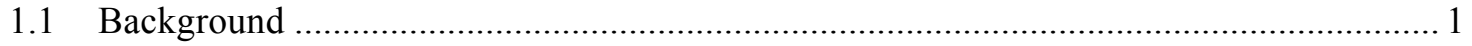

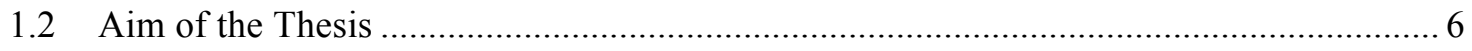

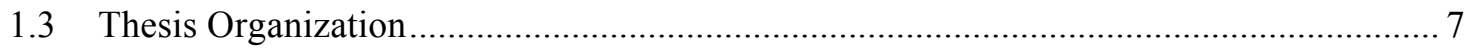

Chapter 2: Comparison of Fluorescence and UV-Vis Spectroscopy for Detection of Polyacrylamide ............................................................................................................................ 11

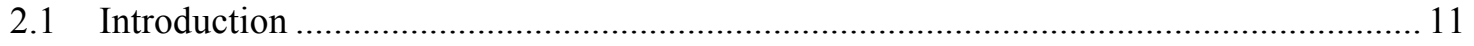

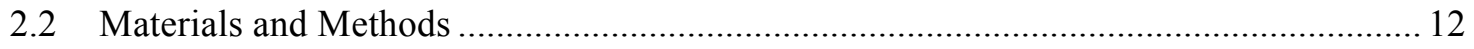

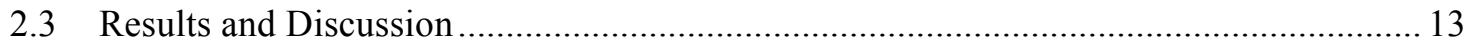

2.3.1 Detection of Zetag8160 Polymer with Fluorescence and UV-Vis Spectroscopy ..... 13

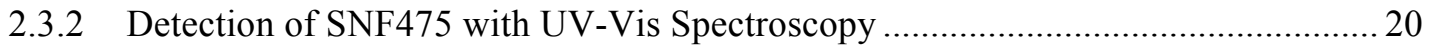

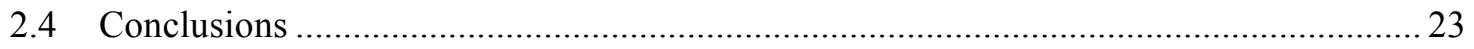

Chapter 3: Effects of pH, Salts, and Inorganic Conditioners on the Detection of

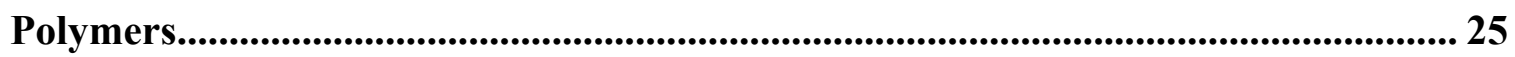

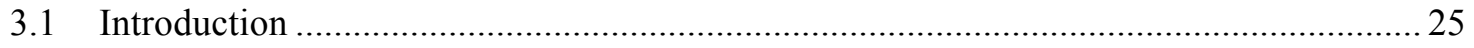

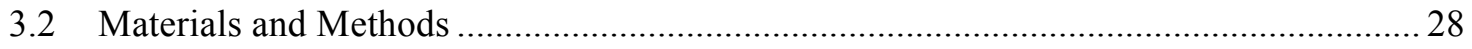

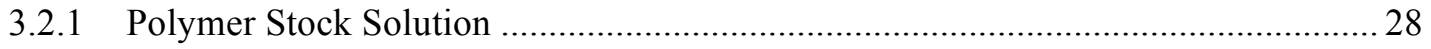




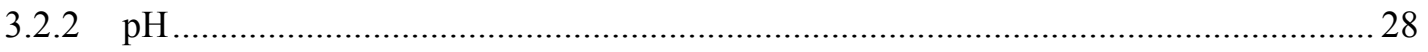

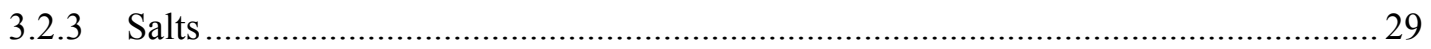

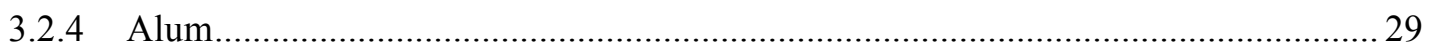

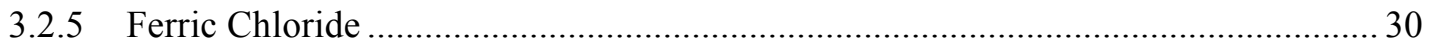

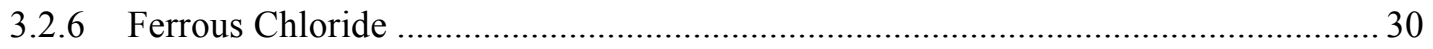

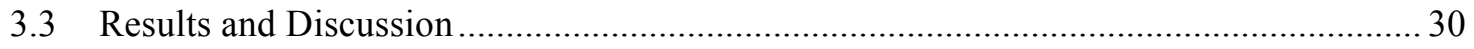

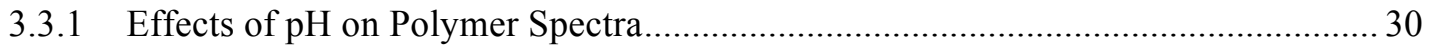

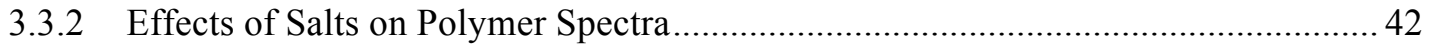

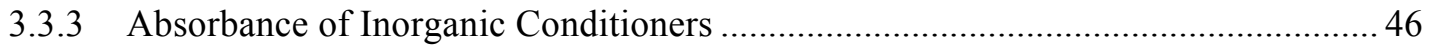

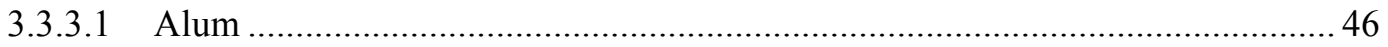

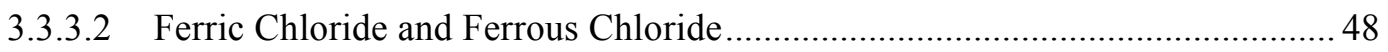

3.3.4 Suggestions to Minimize Interferences ............................................................ 52

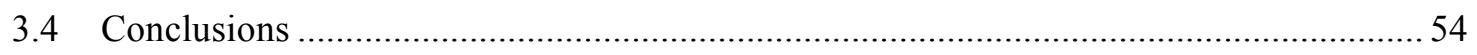

Chapter 4: Comparison of Polymer Detection Methods .......................................... 56

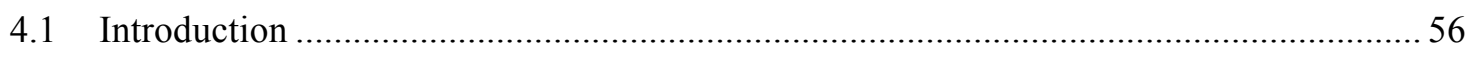

4.1.1 Acidic/Alkaline Hydrolysis (or Dye Complexation) …........................................ 58

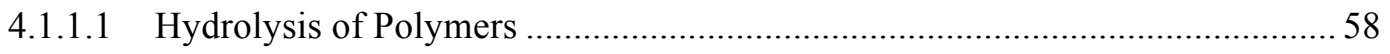

4.1.1.2 Absorbance Measurement of Salts Before and After Polymer Addition via

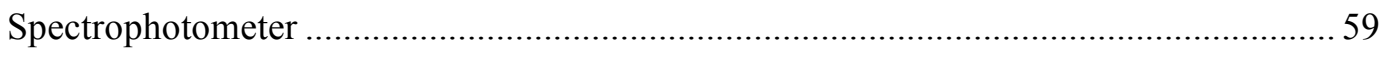

4.1.2 N-Bromination Method and Spectrophotometry of Starch Complex .......................59

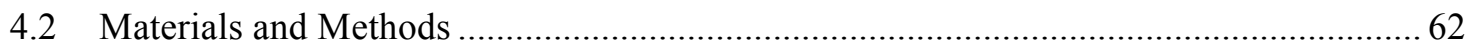

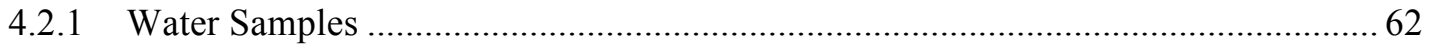

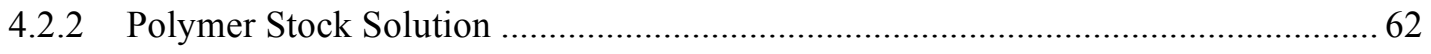

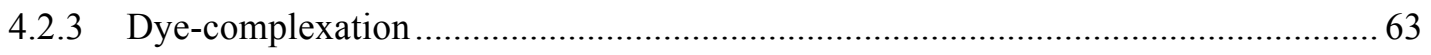

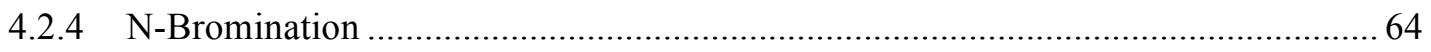

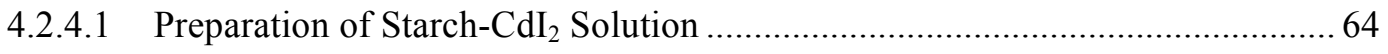




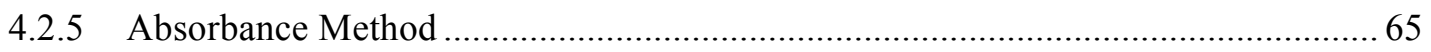

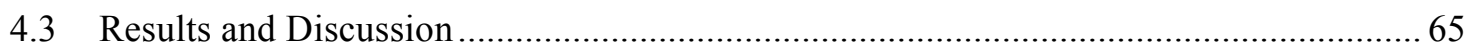

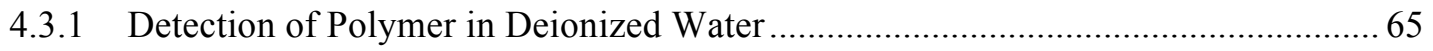

4.3.2 Detection of Polymer in Rideau River Water .......................................................... 70

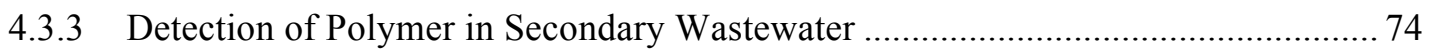

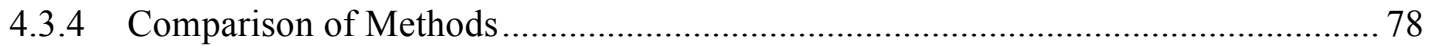

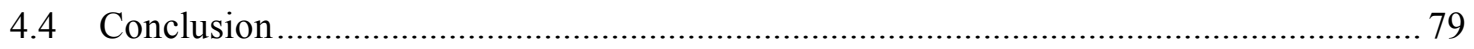

\section{Chapter 5: Determination of Optimum Polymer Dose for Sludge Dewatering Based}

on Residual Polymer Concentration ....................................................................... 80

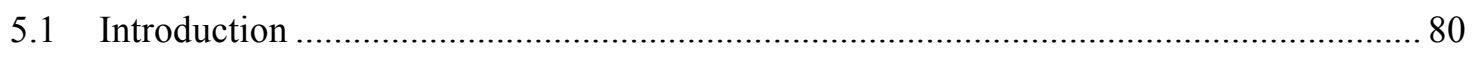

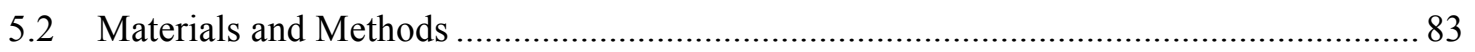

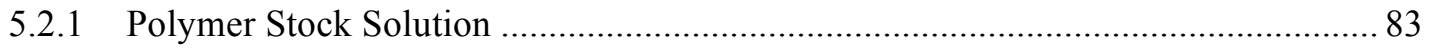

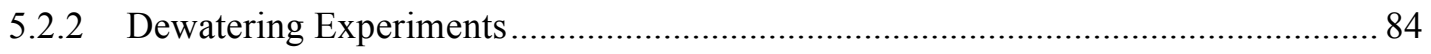

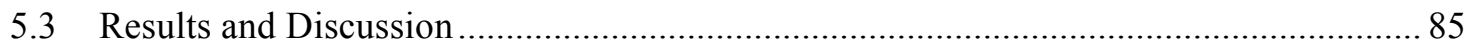

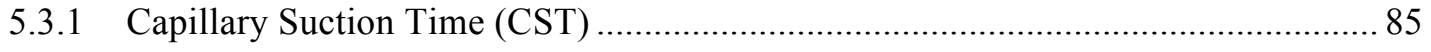

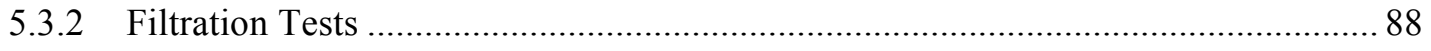

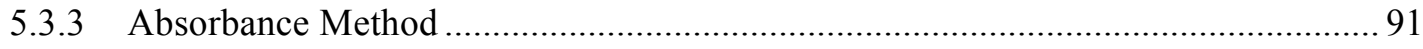

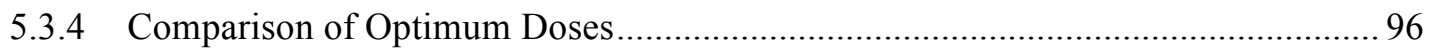

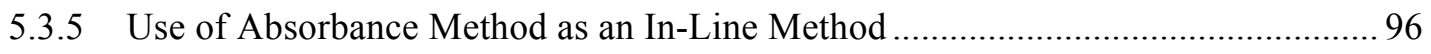

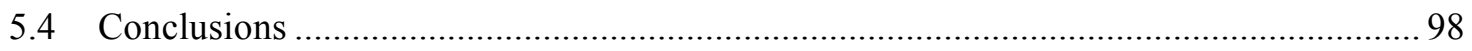

Chapter 6: Conclusions ...................................................................................................... 99

Appendices............................................................................................................................. 101

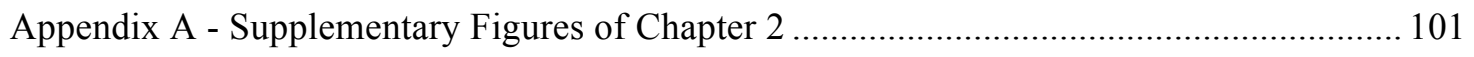

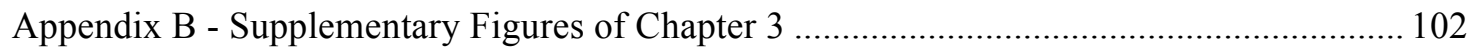




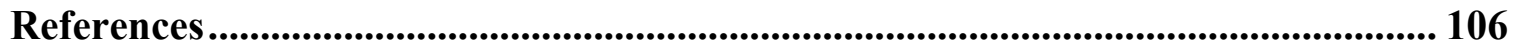




\section{List of Tables}

Table 4.1 Values of MDL for Zetag8160 polymer in different water samples ............ 79

Table 5.1 Optimum doses for all polymers determined by all methods ..................... 96 


\section{List of Figures}

Figure 1.1 Schematic of sludge treatment portion of wastewater treatment plants ......... 1

Figure 2.1 Fluorescence spectra of Zetag8160 polymer (average of three measurements)

Figure 2.2 UV-Vis spectra of Zetag8160 polymer (average of three standards).......... 15

Figure 2.3 Relationship between Zetag8160 concentration and absorbance at $190 \mathrm{~nm}$ in

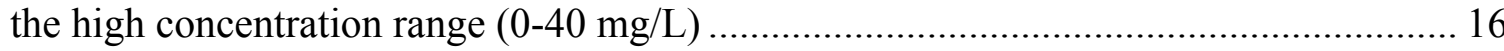

Figure 2.4 Relationship between Zetag8160 concentration and absorbance at $190 \mathrm{~nm}$ in

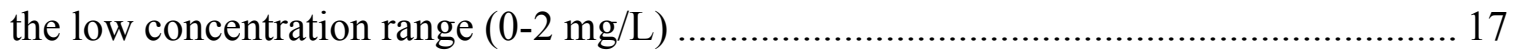

Figure 2.5 UV-Vis spectra of SNF475 polymer (average of three standards)............. 20

Figure 2.6 (a) Linear polymer, (b) Branched polymer, (c) Cross-linked polymer (De

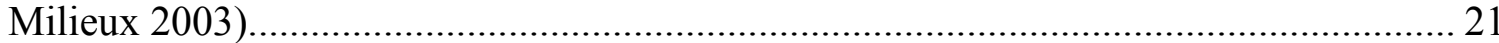

Figure 2.7 Relationship between SNF475 concentration and absorbance at $190 \mathrm{~nm}$ in

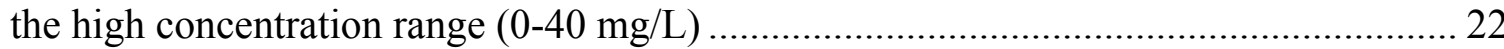

Figure 2.8 Relationship between SNF475 concentration and absorbance at $190 \mathrm{~nm}$ in

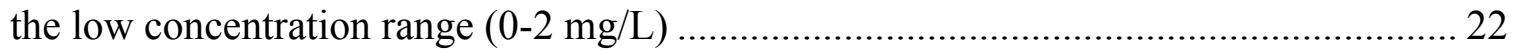
Figure 3.1 Effects of $\mathrm{pH}=3$ on DW, and $5 \mathrm{mg} / \mathrm{L}$ solution of SNF475 in DW (average of

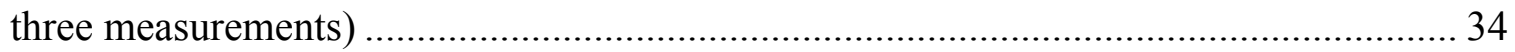
Figure 3.2 Effects of $\mathrm{pH}=5$ on DW, and $5 \mathrm{mg} / \mathrm{L}$ solution of SNF475 in DW (average of

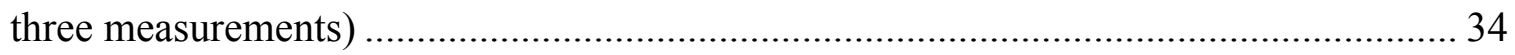

Figure 3.3 Effects of $\mathrm{pH}=6$ on DW, and $5 \mathrm{mg} / \mathrm{L}$ solution of SNF475 in DW (average of

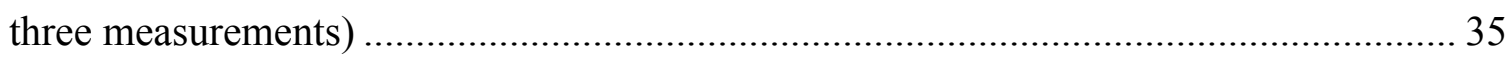


Figure 3.4 Effects of $\mathrm{pH}=7$ on DW, and $5 \mathrm{mg} / \mathrm{L}$ solution of SNF475 in DW (average of

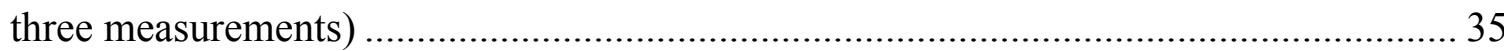

Figure 3.5 Effects of $\mathrm{pH}=8$ on DW, and $5 \mathrm{mg} / \mathrm{L}$ solution of SNF475 in DW (average of

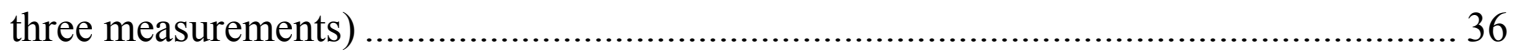

Figure 3.6 Effects of $\mathrm{pH}=9$ on DW, and $5 \mathrm{mg} / \mathrm{L}$ solution of SNF475 in DW (average of

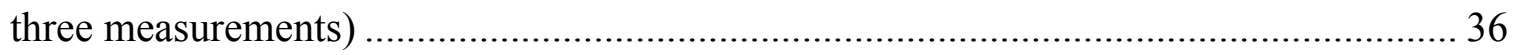

Figure 3.7 Effects of $\mathrm{pH}=11$ on DW, and $5 \mathrm{mg} / \mathrm{L}$ solution of SNF475 in DW (average of three measurements).

Figure 3.8 Effects of $\mathrm{pH}$ on DW, and $5 \mathrm{mg} / \mathrm{L}$ solution of SNF475 in DW at $\lambda=190 \mathrm{~nm}$ (for replicate 1 - average of three measurements)

Figure 3.9 Effects of $\mathrm{pH}$ on DW, and $5 \mathrm{mg} / \mathrm{L}$ solution of SNF475 in DW at $\lambda=190 \mathrm{~nm}$ (for replicate 2 - average of three measurements) 38

Figure 3.10 Effects of $\mathrm{pH}=3.5$ on DW, and $5 \mathrm{mg} / \mathrm{L}$ solution of SNF475 in DW (average of three measurements). 38

Figure 3.11 Effects of $\mathrm{pH}=4$ on DW, and $5 \mathrm{mg} / \mathrm{L}$ solution of SNF475 in DW (average of three measurements). 39

Figure 3.12 Effects of $\mathrm{pH}=4.5$ on DW, and $5 \mathrm{mg} / \mathrm{L}$ solution of SNF475 in DW (average of three measurements). 39 Figure 3.13 Effects of $\mathrm{pH}=9.5$ on DW, and $5 \mathrm{mg} / \mathrm{L}$ solution of SNF475 in DW (average of three measurements) 40 Figure 3.14 Effects of $\mathrm{pH}=10$ on DW, and $5 \mathrm{mg} / \mathrm{L}$ solution of SNF475 in DW (average of three measurements). 40 
Figure 3.15 Effects of $\mathrm{pH}=10.5$ on DW, and $5 \mathrm{mg} / \mathrm{L}$ solution of SNF475 in DW (average of three measurements)

Figure 3.16 Effects of $\mathrm{pH}$ on DW, and $5 \mathrm{mg} / \mathrm{L}$ solution of SNF475 in DW at $\lambda=190 \mathrm{~nm}$ at extreme $\mathrm{pH}$ conditions (average of three measurements). 41

Figure 3.17 Absorbance spectra of $\mathrm{NaCl}$ in $5 \mathrm{mg} / \mathrm{L}$ solution of SNF475 (average of three measurements) 44

Figure 3.18 Relationship between $\mathrm{NaCl}$ concentration and absorbance in $5 \mathrm{mg} / \mathrm{L}$ solution of SNF475 at $\lambda=190 \mathrm{~nm}$ (average of three measurements) 44

Figure 3.19 Absorbance spectra of $\mathrm{CaCl}_{2}$ in $5 \mathrm{mg} / \mathrm{L}$ solution of SNF475 (average of three measurements) 45 Figure 3.20 Relationship between $\mathrm{CaCl}_{2}$ concentration and absorbance in $5 \mathrm{mg} / \mathrm{L}$ solution of SNF475 at $\lambda=190 \mathrm{~nm}$ (average of three measurements) 45

Figure 3.21 Absorbance spectra of alum in DW (average of three measurements) ..... 47

Figure 3.22 Relationship between alum concentration and absorbance in DW at $\lambda=190$ $\mathrm{nm}$ (average of three measurements) 47

Figure 3.23 Relationship between alum concentration (0-100 $\mathrm{mg} / \mathrm{L})$ and absorbance in DW at $\lambda=190 \mathrm{~nm}$ (average of three measurements) 48 Figure 3.24 Absorbance spectra of ferric chloride in DW (average of three measurements) 50

Figure 3.25 Relationship between ferric chloride concentration and absorbance in DW at $\lambda=190 \mathrm{~nm}$ (average of three measurements) 50 Figure 3.26 Absorbance spectra of ferrous chloride in DW (average of three measurements) 51 
Figure 3.27 Relationship between ferrous chloride concentration and absorbance in DW

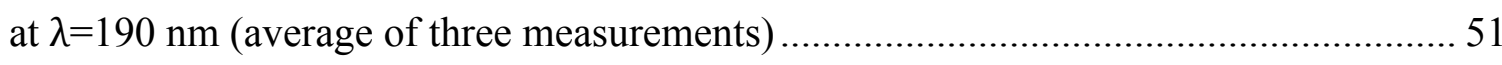

Figure 3.28 Relationship between Zetag8160 concentration and absorbance in DW at

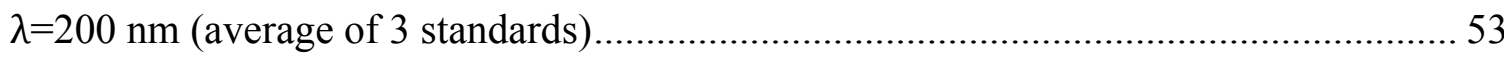

Figure 3.29 Relationship between SNF475 concentration and absorbance in DW at

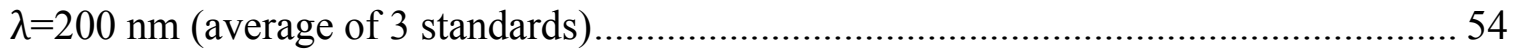

Figure 4.1 Absorbance values of different Zetag8160 concentrations in DW from $\lambda=190$ to $800 \mathrm{~nm}$ using the dye method (average of three measurements) 67

Figure 4.2 Absorbance values of Zetag8160 in DW at $\lambda=646 \mathrm{~nm}$ using the dye method (average of three measurements) 67

Figure 4.3 Absorbance values of different Zetag8160 concentrations in DW from $\lambda=190$ to $800 \mathrm{~nm}$ using the N-Bromination method (average of three measurements) .... 68 Figure 4.4 Absorbance values of Zetag8160 in DW at $\lambda=580 \mathrm{~nm}$ using the NBromination method (average of three measurements) 68

Figure 4.5 Absorbance values of different Zetag8160 concentrations in DW from $\lambda=190$ to $250 \mathrm{~nm}$ using the absorbance method (average of three standards)

Figure 4.6 Absorbance values of Zetag8160 in DW at $\lambda=190 \mathrm{~nm}$ using the absorbance method (average of three standards) 69

Figure 4.7 Absorbance values of different Zetag8160 concentrations in river water from $\lambda=190$ to $800 \mathrm{~nm}$ using the dye method (average of three measurements) 71

Figure 4.8 Absorbance values of Zetag8160 in river water at $\lambda=646 \mathrm{~nm}$ using the dye method (average of three measurements) 
Figure 4.9 Absorbance values of different Zetag8160 concentrations in river water from $\lambda=200$ to $800 \mathrm{~nm}$ using the $\mathrm{N}$-Bromination method (average of three measurements) $\ldots . .72$

Figure 4.10 Absorbance values of Zetag8160 in river water at $\lambda=580 \mathrm{~nm}$ using the NBromination method (average of three measurements) 72

Figure 4.11 Absorbance values of different Zetag8160 concentrations in river water from $\lambda=190$ to $250 \mathrm{~nm}$ using the absorbance method (average of three measurements) .73

Figure 4.12 Absorbance values of Zetag8160 in river water at $\lambda=190 \mathrm{~nm}$ using the absorbance method (average of three measurements) 73

Figure 4.13 Absorbance values of different Zetag8160 concentrations in wastewater from $\lambda=190$ to $800 \mathrm{~nm}$ using the dye method (average of three measurements)...... 75 Figure 4.14 Absorbance values of Zetag8160 in wastewater at $\lambda=646 \mathrm{~nm}$ using the dye method (average of three measurements) 75

Figure 4.15 Absorbance values of different Zetag8160 concentrations in wastewater from $\lambda=200$ to $800 \mathrm{~nm}$ using the N-Bromination method (average of three measurements) 76

Figure 4.16 Absorbance values of Zetag8160 in wastewater at $\lambda=580 \mathrm{~nm}$ using the NBromination method (average of three measurements) 76

Figure 4.17 Absorbance values of different Zetag8160 concentrations in wastewater from $\lambda=190$ to $250 \mathrm{~nm}$ using the absorbance method (average of three measurements) .77 Figure 4.18 Absorbance values of Zetag8160 in wastewater at $\lambda=190 \mathrm{~nm}$ using the

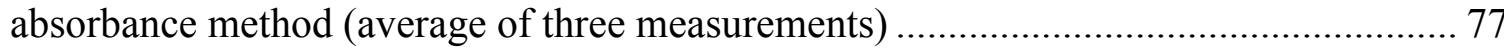

Figure 5.1 Mechanism of a CST test (adopted from Ormeci, 2013) .......................... 85

Figure 5.2 Zetag8160 CST test (average of three measurements) ............................. 86 
Figure 5.3 SNF475 CST test (average of three measurements)................................ 87

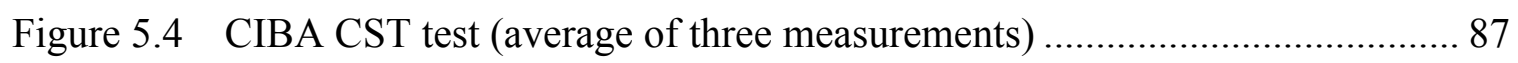

Figure 5.5 Filtration test apparatus (adopted from http://scialert.net/) ....................... 88

Figure 5.6 Zetag8160 filtration test ............................................................ 90

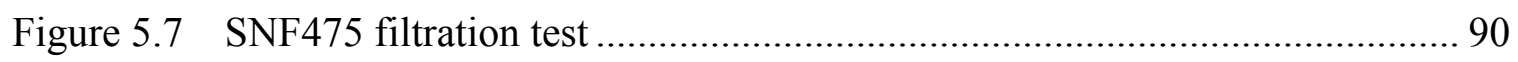

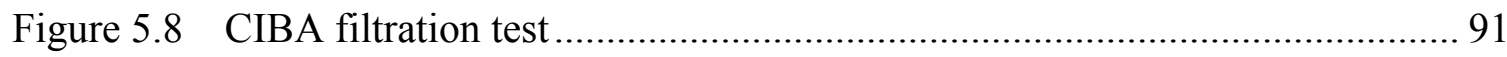

Figure 5.9 Zetag8160 absorbance after dilution at 1v(filtrate): 9v(DW) (average of

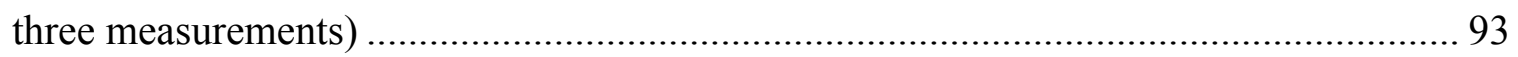

Figure 5.10 Zetag8160 absorbance test (average of three measurements) .................. 93

Figure 5.11 SNF475 absorbance test after dilution at 1v(filtrate): 9v(DW) (average of

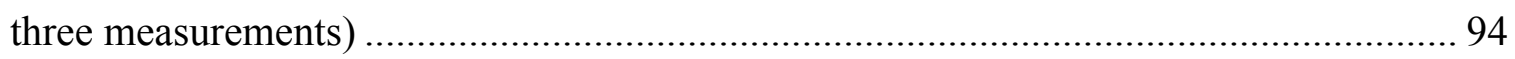

Figure 5.12 SNF475 absorbance test (average of three measurements) ...................... 94

Figure 5.13 CIBA absorbance test from after dilution at $1 \mathrm{v}$ (filtrate): $9 \mathrm{v}(\mathrm{DW})$ (average

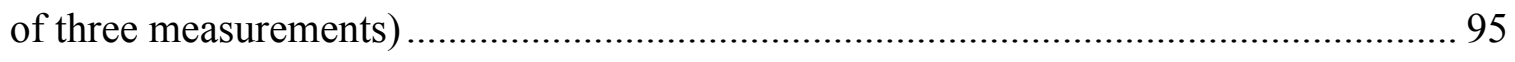

Figure 5.14 CIBA absorbance test (average of three measurements)......................... 95 Figure 5.15 Example of a control loop for automatic adjustments in dosage of polymer

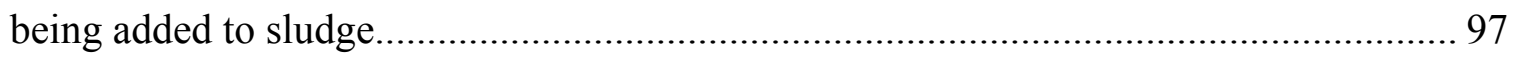

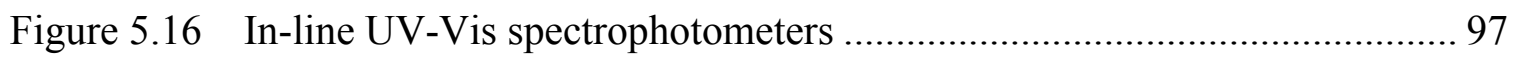

Figure A.1 A snap shot of the automated excel sheet from chemiasoft website (Chemiasoft.com, accessed February 2014) for MDL calculation using SNF475 polymer

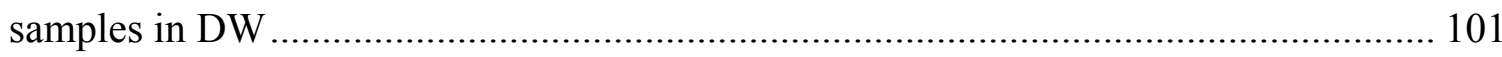

Figure B.1 Effects of $\mathrm{pH}=3$ on DW, and $5 \mathrm{mg} / \mathrm{L}$ solution of SNF475 in DW (average of

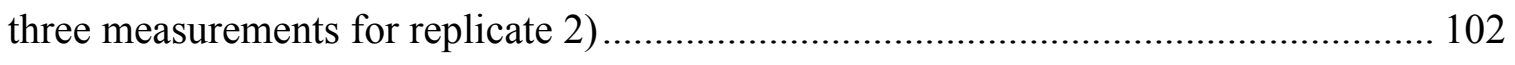


Figure B.2 Effects of $\mathrm{pH}=5$ on DW, and $5 \mathrm{mg} / \mathrm{L}$ solution of SNF475 in DW (average of

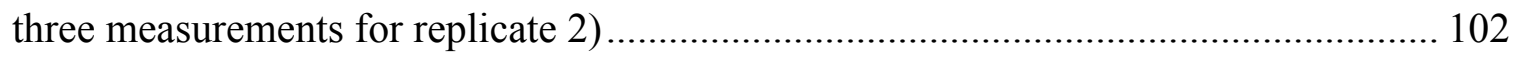

Figure B.3 Effects of $\mathrm{pH}=6$ on DW, and $5 \mathrm{mg} / \mathrm{L}$ solution of SNF475 in DW (average of three measurements for replicate 2) 103

Figure B.4 Effects of $\mathrm{pH}=7$ on DW, and $5 \mathrm{mg} / \mathrm{L}$ solution of SNF475 in DW (average of three measurements for replicate 2) 103

Figure B.5 Effects of $\mathrm{pH}=8$ on DW, and $5 \mathrm{mg} / \mathrm{L}$ solution of SNF475 in DW (average of three measurements for replicate 2). 104 Figure B.6 Effects of $\mathrm{pH}=9$ on DW, and $5 \mathrm{mg} / \mathrm{L}$ solution of SNF475 in DW (average of three measurements for replicate 2). 104 Figure B.7 Effects of $\mathrm{pH}=11$ on DW, and $5 \mathrm{mg} / \mathrm{L}$ solution of SNF475 in DW (average of three measurements for replicate 2) 105 


\section{List of Appendices}

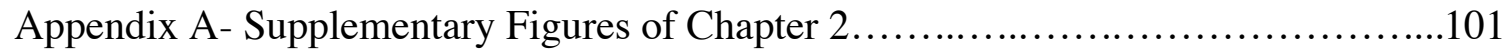

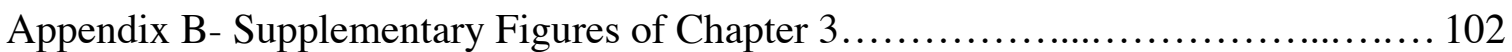




\section{Chapter 1: Introduction}

\subsection{Background}

Treatment and management of sludge, often referred to as "biosolids", contributes to $25-50 \%$ of the total operational budget of a wastewater treatment plant. Many actions such as production of energy (for example production of methane $\left(\mathrm{CH}_{4}\right)$ gas from anaerobic digestion of sludge), and/or preparation of class A or B sludge for land application have been taken by wastewater treatment plants in order to reduce some of the costs associated with sludge treatment. Wastewater treatment plants also try to counterbalance these costs by optimizing the sludge treatment processes and making the overall treatment more feasible. The focus of this study is also to optimize sludge conditioning and dewatering and to reduce the cost of sludge management and disposal.

A brief description of sludge treatment process (shown in Figure 1.1 below) is provided before presenting further details on the study.

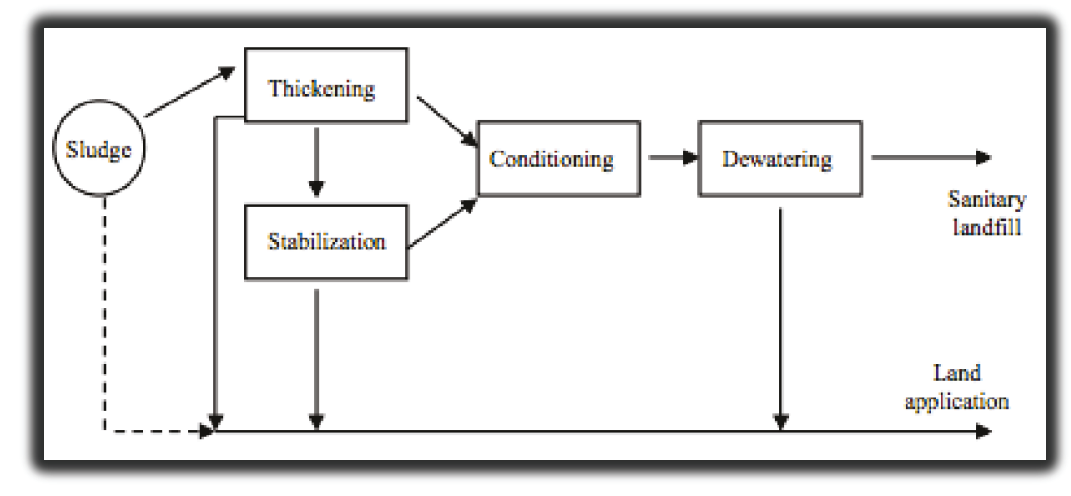

Figure 1.1 Schematic of sludge treatment portion of wastewater treatment plants

As shown in Figure 1.1 above, sludge treatment is comprised of several steps.

Firstly, the primary and secondary sludge collected from primary and secondary clarifiers in wastewater treatment plants are thickened. The objective of the thickening step is to 
reduce some of the water content of the sludge, which results in reduction of the volume of the downstream units. Depending on the type of the sludge (primary, secondary, or mixture of both), different types of thickeners can be selected. Usually gravity thickener is used for primary sludge or mixed primary and waste activated (secondary) sludge since these solids are heavy and easy to settle. Other thickener types such as dissolved air floatation (DAF) or centrifuge can be used for thickening of lighter solids such as waste activated sludge.

Once the sludge is thickened, it is digested, either aerobically or anaerobically, in order to kill pathogens, reduce organic matter and eliminate potentially odor-causing material.

After stabilization, sludge is conditioned via inorganic coagulants such as alum, ferric chloride, ferrous chloride, or via synthetic polymers prior to dewatering. Conditioning destabilizes the colloidal materials and causes the small particles to agglomerate into larger settleable flocs (Amuda and Amoo 2007). The coagulation/flocculation process helps solids release their water, which is then removed once sludge gets to the mechanical dewatering units such as centrifuges or belt press filters.

Based on the characteristics and charge of the solid particles in digested sludge, there is an optimum conditioner (usually synthetic polymer) dose that can result in better dewatering, and therefore higher cake solids. Generally, the solid particulates in municipal wastewater are of negative charge, once cationic polymer is introduced into the sludge, it neutralizes the negative charge of the solid particulates. The neutralized particles attach to the branches of polymer and since they are neutralized and do not repel 
one another anymore, they bridge together and produce flocs until optimum polymer dose is reached. At doses higher than that of optimum polymer dose, the increase of positively charged polymers in the solution will have adverse effects on floc formation as well as floc structure.

Conditioning and dewatering are specifically important for reduction of the costs associated with handling, and disposal of sludge; as sludge transportation, landfill tipping fees, and incineration costs are very expensive, and greater moisture removal (dewatering) from the sludge results in lower sludge volume, which can have substantial decrease in hauling, landfill tipping fees, and incineration costs. Due to this reason, there is an interest in optimization of sludge dewatering process in wastewater treatment plants.

Benitez et al. (1994) tried optimizing dewatering of primary sludge from a combined industrial and municipal wastewater by promoting production of a porous and incompressible cake that has lower resistance to filtration, and does not deform under pressure. This was done by adding a skeleton builder (e.g., fly ash from a municipal sludge incinerator, cement kiln dust, and bagasse) along with the polymer to condition sludge. These skeletons were inerts that formed a permeable and rigid lattice structure with sludge that remained porous under pressure. Once these skeleton builders and polymer were added in optimum proportions, they resulted in 580\%, 96\%, 1260\% increase in the net sludge solid yield with fly ash, cement kiln dust, and bagasse respectively.

Lee and Liu (2000) enhanced dewatering of waste activated sludge from a synthetic fiber plant by using dual electrolytes (a combination of cationic and non-ionic polymers). This treatment involved pre-conditioning of sludge via cationic polymer 
followed by a non-ionic one. Dual conditioning showed reversed zeta potentials in contrast to zero zeta potential values for use of single (cationic) polyelectrolyte, indicating the need for lower conditioner dosage for optimal dewatering. Addition of cationic polymer caused the formation of primary flocs, and further addition of non-ionic polymer formed an extended conformation of polyelectrolytes by hydrogen bonding on the loops and tails of cationic polymer, resulting in thicker flocs with less free water.

The studies of Benitez et al. (1994) as well as Lee and Liu (2000) are focused on the nature of conditioning additives. However, when the conditioning additives are specified, the dosage parameters to assure optimal dewatering must not only be established but also be maintained (Dentel, Abu-Orf and Walker 2000). This task is complicated due to the daily, seasonal, and annual variations in the colloidal characteristics of sludge (Dentel, Abu-Orf and Walker 2000). This challenge is further complicated by changes in conditioner characteristics between batches (Dentel, Abu-Orf and Walker 2000). Commonly, the response to such variability is routine conditioner overdosing, since the deterioration in process efficiency is less than that observed with underdosing (Dentel, Abu-Orf and Walker 2000). Therefore, the control of polymer dose on a regular basis is crucial.

Dentel et al. (2000) used several approaches such as Streaming Current Detector (SCD) which measures the zeta potential associated with the particles, viscosity, and some other rheological properties for control of polymer at the optimum dose. They used indicators such as Capillary Suction Time (CST) to show that the optimum polymer dose for dewatering of sludge corresponds to a streaming current of about zero, and used that towards controlling the polymer feed pump. 
Furthermore, Dentel et al. (2000) used findings from Campbell et al. (1978) and tried to adjust conditioner flow rate based on rheogram curves of sludge. Campbell et al. (1978) demonstrated that upon increase in the shear rate applied to sludge, its initial shear stress increases. This initial increase in shear stress is dependent on the degree of conditioner applied to the sludge. Even though after further increase in shear rate, the shear stress of the sludge drops due to the disruption of the flocculated matrix, the initial increase in shear stress for optimally conditioned sludge is higher compared to nonoptimally conditioned sludge.

Dentel et al. (2000) also used the research work of Christensen et al. (1993) on the viscosity of filtrate or centrate of anaerobically digested sludge to optimize polymer addition to sludge. Christensen et al. (1993) observed a lower viscosity for filtrate/centrate of anaerobically digested sludge when it was optimally conditioned. Deviations from optimal conditioner dosage resulted in higher viscosities at doses lower, and higher than that of optimum dose, due to existence of small solid particles, and unreacted polymer chains respectively.

Eisenlauer and Horn (1985) also tried optimizing dewatering process through rapid in-situ methods of assessing the degree of flocculation in streaming systems. They developed a microcomputer-controlled laser optical flocculation sensor for this purpose that can measure the floc size in a flowing medium. The device is a combination of a flow-through cell, fiber-optic laser-diode, as well as a microcomputer. Once the flocs enter the flow-through cell, they block the light that is being captured by the fiber-optic laser-diode. The light blockage signals provided by the fiber-optic laser-diode are then sent to the microcomputer that can give an estimate of the floc size based on the root 
mean square analysis of fluctuating light blockage signals from the fiber-optic laser-diode assembly. Together with a control loop on the conditioner pump, this signal can provide adjustments in conditioner flow rate for better dewatering.

\subsection{Aim of the Thesis}

Likewise to the studies of Dentel et al. (2000) as well as Eisenlauer and Horn (1985) mentioned in the last section, the purpose of this study is to define the dosage and nature of conditioning additives to the digested sludge and to maintain it at the optimum level. However, unlike the studies of Dentel et al. (2000) and Eisenlauer and Horn (1985) that use rheology of sludge, floc characteristics, and/or centrate/filtrate viscosity to control polymer feed rate, this research work aims to directly measure the residual polymer concentration in centrate/filtrate after dewatering and correlate that to the optimum polymer dose, which would enable adjustments to the polymer dose that is being added to sludge. In order to achieve this, a quick and simple method that can be used in-line and real-time at treatment plants needs to be developed first which also is in the scope of this thesis.

There are a number of methods reported in literature that can measure the concentration of polyacrylamide polymers that are the most commonly used polymers at treatment plants. A thorough review of these methods is provided in Chapter 2 and 4 of this thesis. These methods, however, are very time consuming, labor extensive, and usually incorporate the use of other reagents that form a complex with polyacrylamide. Therefore, they cannot be used in-line and real-time. In this thesis, it was of interest to eliminate the additional steps and develop a direct method that can be used in-line and 
real-time to achieve optimization of polymer dose as well as automation of digested sludge dewatering in the near future.

The more specific objectives of this research can be listed as follows:

1. Evaluate the potential of UV-Vis and fluorescence spectrophotometry for the detection and measurement of polyacrylamide polymers.

2. Develop a quick, simple, and sensitive method that can measure polymer concentration in water, and wastewater.

3. Compare the performance of this method with other methods reported in the literature and establish detection limits.

4. Investigate the potential interferences and the effect of $\mathrm{pH}$, salts and other wastewater treatment plant chemicals on the method.

5. Use the method to measure residual polymer concentration in filtrate and centrate after sludge dewatering.

6. Establish a relationship between the residual polymer concentration in filtrate/centrate and the optimum polymer dose required to condition the digested sludge.

\subsection{Thesis Organization}

\section{Chapter One:}

Chapter one provides an introduction to the thesis topic, highlights the need for the study and presents the objectives of this work.

\section{Chapter Two:}

In Chapter 2, different concentrations of polymers were prepared in deionized water, and their intensities and absorbances were scanned over wavelengths of 190-800 
$\mathrm{nm}$ using fluorescence and UV-Vis spectrophotometer respectively, to verify whether these polymers could be directly detected without the need for an intermediate step or not.

The results that are reported in Chapter 2 showed an apparent difference between the absorbance of deionized water, and deionized water samples with increasing polymer doses, with the peak of absorbance occurring at $190 \mathrm{~nm}$ for each solution, when measured via $\mathrm{UV}-\mathrm{V}$ is spectrophotometer. Furthermore, the absorbance values at $190 \mathrm{~nm}$ followed a linear trend when plotted against concentration of polymers that were added in deionized water.

Fluorescence spectrophotometry, however, failed to provide any meaningful outcome, as the intensities of differently dosed deionized water solutions with polymer were randomly scattered around the value of zero between the wavelengths of 190-800 $\mathrm{nm}$.

\section{Chapter Three:}

Following the results from Chapter 2, the interference of $\left[\mathrm{H}^{+}\right],\left[\mathrm{OH}^{-}\right]$ions $(\mathrm{pH})$, along with commonly used chemicals (alum, ferric chloride, and ferrous chloride) and salts (sodium chloride and calcium chloride) on the absorbance spectra of polymers were investigated in Chapter 3 of this thesis.

The interferences were scrutinized either by measuring the absorbance of increasing concentrations of the salts, ions and/or chemicals in deionized water or in 5 $\mathrm{mg} / \mathrm{L}$ polymer samples at wavelengths of $190-800 \mathrm{~nm}$. Alum interfered with the polymer spectra at high concentrations and other chemicals, ions, and salts showed interference starting from low concentrations. 


\section{Chapter Four:}

In Chapter 4, the sensitivity for direct detection of polymer using UV-Vis spectroscopy, named the "absorbance method" was put to the test against two other methods of N-Bromination, and amide hydrolysis from literature.

The sensitivities of the three different methods were compared using statistical analysis and calculation of the Method Detection Limit (MDL), which is the lowest concentration that can lead to detection with statistical significance.

The absorbance method not only measured polymer in lower doses in deionized water samples compared to the other two methods, but also was able to detect polymer in lower concentrations in other environmental samples such as Rideau River water and secondary wastewater from ROPEC, the City of Ottawa's wastewater treatment plant.

\section{Chapter Five:}

In Chapter 5, the potential use of the developed method towards improving the conditioning and dewatering processes was investigated.

For this purpose, anaerobically digested sludge from ROPEC was collected and jar tested with different polymer concentrations. The jar tested samples were then filtered and the absorbance of the filtrate was monitored over wavelengths of 190-800 nm using UV-Vis spectroscopy. It was found that the absorbance (at $190 \mathrm{~nm}$ ) of the sludge filtrate at the optimum polymer dose was lowest. Using indicators such as Capillary Suction Time (CST), and filtration tests the optimum polymer dose found with UV-Vis spectroscopy was confirmed. The sample with lowest absorbance corresponded to the same sample with lowest CST, and highest filtrate volume. 
Therefore, a relationship between the residual polymer concentration and the optimum polymer dose was successfully established. The results showed that residual polymer concentration could be used as an indicator of the degree to which sludge is being conditioned, and can be utilized to directly control the feed rate of the polymer to sludge. 


\section{Chapter 2: Comparison of}

\section{Fluorescence and UV-Vis Spectroscopy}

\section{for Detection of Polyacrylamide}

\subsection{Introduction}

Synthetic polymers have gained a lot of attention as conditioners in wastewater treatment plants due to the fact that they do not increase the amount of sludge produced, are very efficient and are generally non-toxic depending on their type (Daughton 1988). Moreover, usually small amount of polymer is added as coagulant compared to coagulants such as lime and ferric/ferrous chloride.

The most common polymer that has a vast use in wastewater treatment plants as well as many other industries is polyacrylamide $\left[-\mathrm{CH}\left\{\mathrm{C}(=\mathrm{O})-\mathrm{NH}_{2}\right\}-\mathrm{CH}_{2}-\right]_{\mathrm{n}}$ that consists of monomers of acrylamide $\mathrm{CH}_{2}=\mathrm{CH}-\mathrm{C}(=\mathrm{O})-\mathrm{NH}_{2}$. Unreacted acrylamide is harmful and has a very high solubility in water. Fortunately, it quickly biodegrades in waters with high bacterial population and has a half-life of about 2 days in soil-waters. In drinking waters, however, due to the low concentration of bacteria, acrylamide is not biodegraded and is highly persistent (Daughton 1988). Even though it was mentioned that polymers in general are non-toxic, polyacrylamide has been known to be carcinogenic and may cause severe neuro- and geno-toxicity problems mainly due to the existing unreacted acrylamide monomers (Daughton 1988). 
So far, there exists no standardized method in order to directly measure the concentration of polyacrylamide that is being emitted into the environment. In addition, the existing methods are either too sophisticated or the simpler ones do not have the required sensitivity. Usually, the discharge of polymers into surface waters is monitored indirectly and through calculations of how much polymer is added and the manufacturer's guide on the percentage of unreacted acrylamide per unit mass of the polymer. Therefore, there is a need for quick, simple, and accurate methods that can be relied upon for direct detection and quantification of polyacrylamide.

The goal of this chapter was therefore to investigate the direct use of UV-Vis and fluorescence spectroscopy for detection and quantification of polyacrylamide polymers in deionized water samples without the use of chemicals or intermediate steps.

\subsection{Materials and Methods}

Polymer stock solutions of $500 \mathrm{mg} / \mathrm{L}$ or $0.05 \%$ were prepared as follows: in two plastic weighing dishes, $0.5 \mathrm{~g}$ of Zetag8160 (polyacrylamide, medium-high cationic charge, high molecular weight, granular solid form) and $0.5 \mathrm{~g}$ of SNF475 (polyacrylamide, high charge density, ultra-high molecular weight, granular solid form) polymers were weighted using a scale (SI-114, Denver Instruments, Canada) and decanted into two $1 \mathrm{~L}$ glass beakers along with $1 \mathrm{~L}$ of deionized water in each beaker. The mixtures were stirred using a jar tester (Phipps and Bird, USA) at $200 \mathrm{rpm}$ for 5 minutes followed by 55 minutes at $125 \mathrm{rpm}$ and were allowed to rest for 60 minutes prior to usage (Gibbons and Ormeci 2013). 
After the resting time of the polymer stock solutions, in $50 \mathrm{~mL}$ centrifuge tubes, three replicates of each polymer standard solutions of $0,0.01,0.05,0.1,0.5,1,2,4,6,8$, $10,20,30$, and $40 \mathrm{mg} / \mathrm{L}$ were prepared using the stock solutions.

The intensity and absorbance of the prepared samples were then measured via Fluorescence (Cary Eclipse spectro-fluorimeter, Varian Inc., Canada), and UV-Vis spectrophotometer (Cary 100 Bio UV-Vis Spectrophotometer, Varian Inc./Aligant Technologies, Canada) using glass cuvettes (Hellma Canada Ltd., Canada) and recorded using Scan software (Cary WinUV, Varian Inc., Canada) over a range of 200-800 $\mathrm{nm}$ for fluorescence and over a range of 190-250 $\mathrm{nm}$ for UV-Vis spectroscopy.

\subsection{Results and Discussion}

\subsubsection{Detection of Zetag8160 Polymer with Fluorescence and UV-Vis Spectroscopy}

The results from Scan software for Zetag8160 polymer are depicted in Figures 2.1, and 2.2 for fluorescence and UV-Vis spectroscopy, respectively. As seen in Figure 2.1, the fluorescence intensity of the prepared samples of Zetag8160 do not adopt any trend while plotted against wavelength and are randomly distributed around $\mathrm{X}$ axis. This can be explained by the fact that polymers of acrylamide consist of non-aromatic molecules, which do not usually fluoresce (Lakowicz 2006) and due to this reason they adopt an average intensity of 0 a.u. over the wavelengths of 200-800 nm as shown in Figure 2.1.

Hendrickson and Neuman (1984) as well as Arryanto and Bark (1991) used

fluorescence spectroscopy for detection of polyacrylamide polymers but they used two 
intermediate reactions to convert polyacrylamide to a more fluorescing compound which then could be detected via fluorescence spectroscopy.

Hendrickson and Neuman (1984) could detect polyacrylamide in dosages as low as $20 \mu \mathrm{g} / \mathrm{L}$ by carrying out chemical reactions to convert polyacrylamide to its amine derivative and then created a highly fluorescing compound in the presence of ophthalaldehyde and 2-mercaptoethanol. Being very sensitive, the draw back of their method was the very slow rates of about two days for carrying out the first step of the reaction. Arryanto and Bark (1991) then optimized the first step of the reaction to minimize the reaction time from two days to about two hours. However, detection limit was increased to a value of $1 \mathrm{mg} / \mathrm{L}$ as opposed to $20 \mu \mathrm{g} / \mathrm{L}$ for the work of Hendrickson and Neuman (1984).

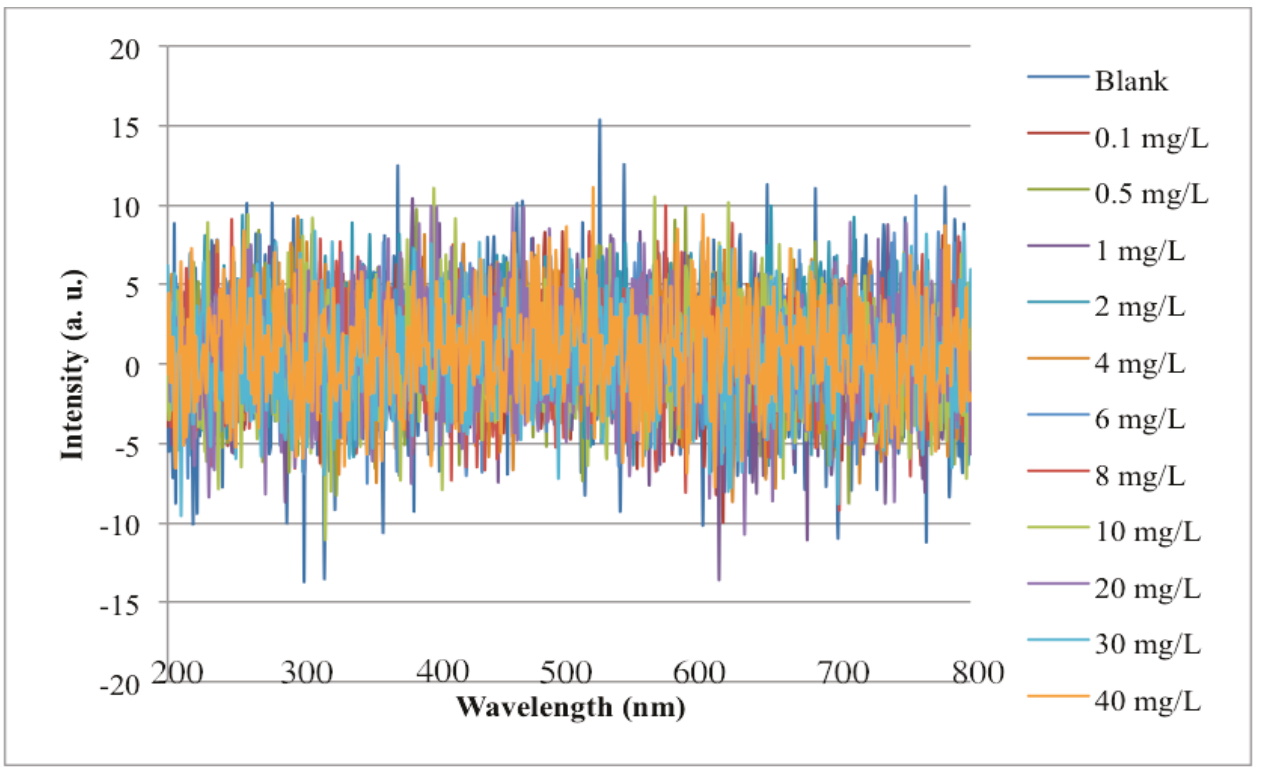

Figure 2.1 Fluorescence spectra of Zetag8160 polymer (average of three measurements)

Based on the data in Figure 2.1, direct fluorescence spectroscopy, without any intermediate steps or reactions, was not found to be useful for the purposes of this study. Therefore, further experimental work utilizing fluorescence spectroscopy was stopped. 
As opposed to fluorescence, however, the absorbance of Zetag8160 samples versus wavelength adopted a curvature trend with a maximum at $190 \mathrm{~nm}$ that gradually decreased to around zero as wavelength was increased to $250 \mathrm{~nm}$ (Figure 2.2). Furthermore, as shown in Figure 2.2, the absorbance at each Zetag8160 concentration appeared to be proportional to polymer concentration and as the concentration was increased, the absorbance curves seemed to shift up at lower wavelengths of 190-220 nm, and stay close to zero from 220 to $250 \mathrm{~nm}$.

Therefore UV-Vis spectroscopy appeared to be very promising for detection of polyacrylamides, especially at $\lambda=190 \mathrm{~nm}$ where the differences between absorbance of each polymer concentration were more significant compared to other wavelengths. Due to this reason, $\lambda=190 \mathrm{~nm}$ was chosen in order to increase the method sensitivity as polymer dose in deionized water was increased.

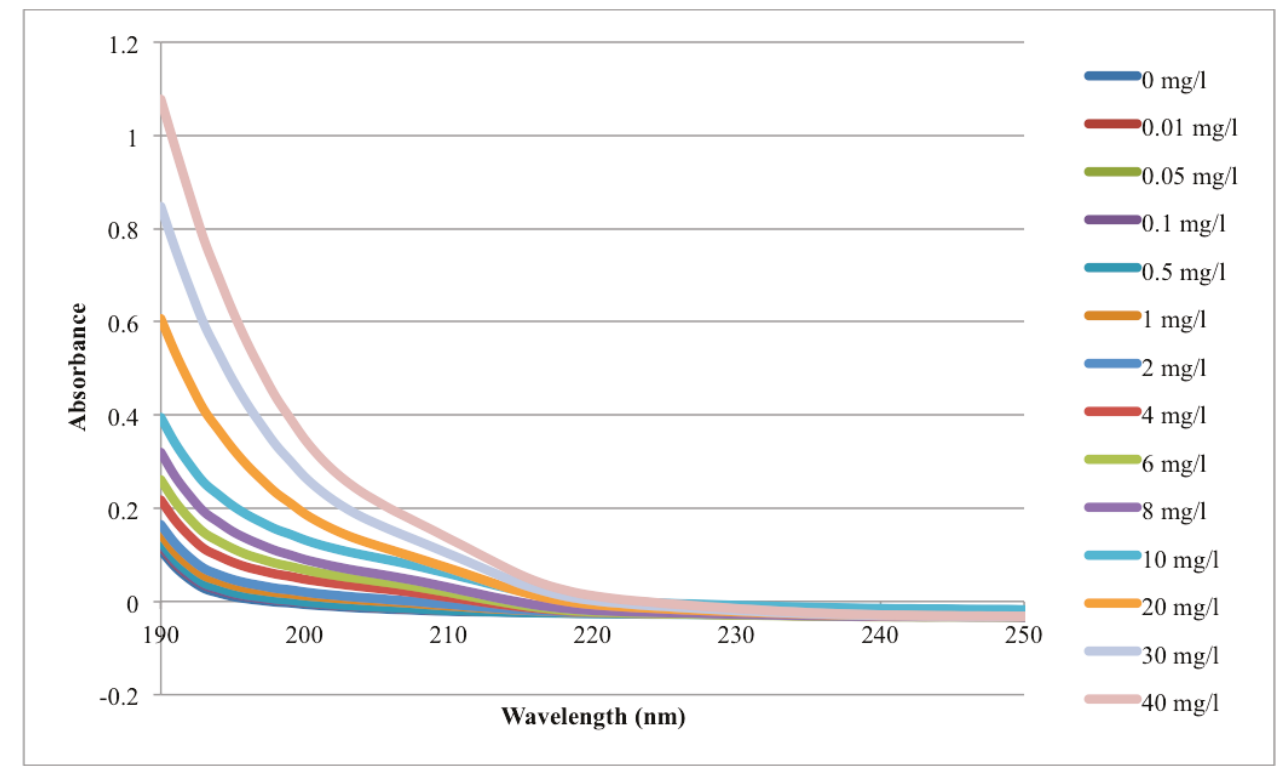

Figure 2.2 UV-Vis spectra of Zetag8160 polymer (average of three standards)

As depicted in Figure 2.3, the absorbance of Zetag8160 polymer at $190 \mathrm{~nm}$ adopts a perfect linear relationship against concentration. Furthermore, as shown in Figure 2.4, 
which shows the relationship between concentration and absorbance, it is clear that UVVis spectroscopy can detect very small increases in polymer concentrations in the $0-2$ $\mathrm{mg} / \mathrm{L}$ range, and possibly as low as $0.01 \mathrm{mg} / \mathrm{L}$ in deionized water (Figure 2.4). However, at very low doses (less than $0.2 \mathrm{mg} / \mathrm{L}$ ), the linear trend is not as strong as high doses (Figure 2.3), indicating that the method may have reached its detection limit at these concentrations.

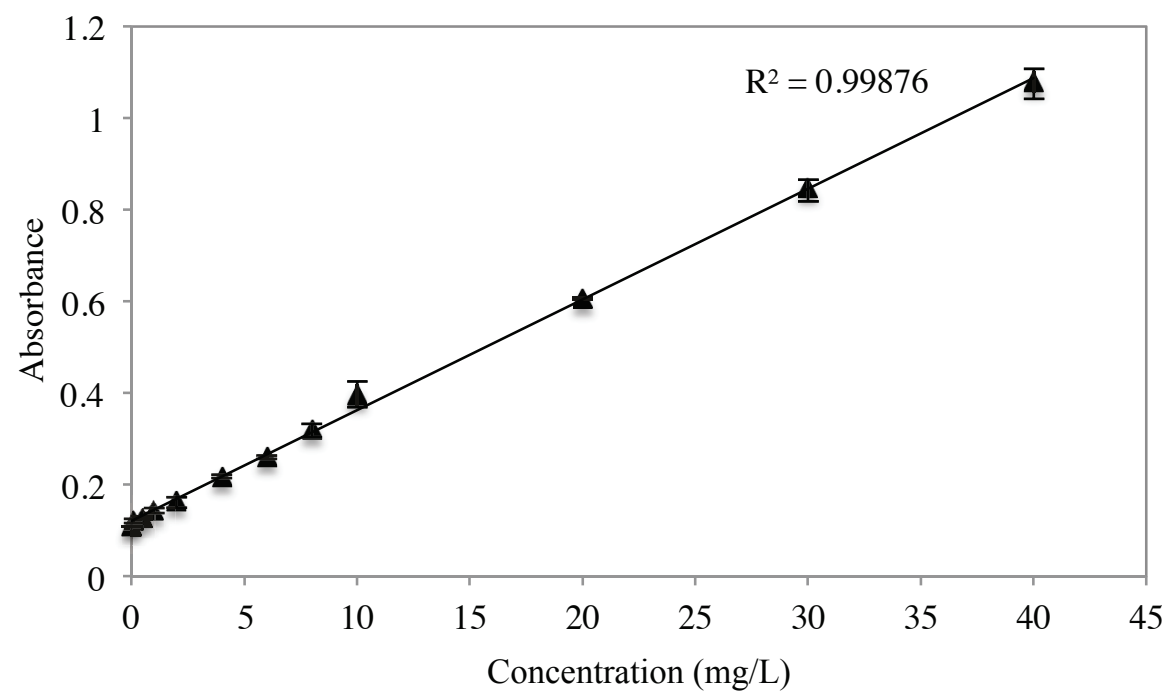

Figure 2.3 Relationship between Zetag8160 concentration and absorbance at $190 \mathrm{~nm}$ in the high concentration range $(0-40 \mathrm{mg} / \mathrm{L})$ 


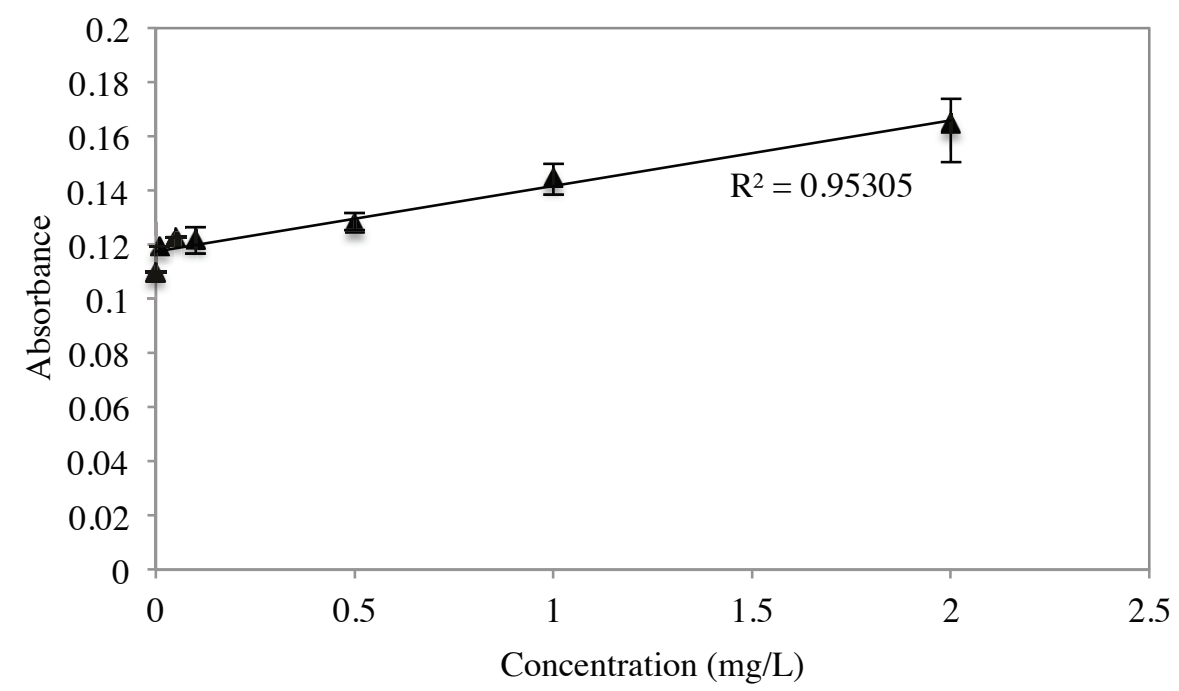

Figure 2.4 Relationship between Zetag8160 concentration and absorbance at $190 \mathrm{~nm}$ in the low concentration range $(0-2 \mathrm{mg} / \mathrm{L})$

Detection Limit, or Method Detection Limit (MDL) is the limit, at which a given analytical procedure (in this case the absorbance method) may be relied upon to lead to detection (in this case the detection of polymer concentration within a sample based on its absorbance) (Hubaux and Vos 1970). Even though, as mentioned before, UV-Vis spectroscopy captures a difference between the absorbance of a blank sample and a sample with $0.01 \mathrm{mg} / \mathrm{L}$ of polymer; $0.01 \mathrm{mg} / \mathrm{L}$ sample may not necessarily be the detection limit of the method. MDL is strongly dependent on the quality of fitting a linear line to the concentration of each standard versus its corresponding measurement from the instrument, and as shown in Figure 2.4 the points corresponding to 0.01, 0.05, and 0.1 $\mathrm{mg} / \mathrm{L}$ do not fall perfectly on the linear regression line relating concentration to absorbance. The significance of this is that unknown samples with concentrations ranging from 0 to $0.1 \mathrm{mg} / \mathrm{L}$, cannot be distinguished from each other, and statistical analysis 
should be used to define MDL that is the boundary between the sample that could be determined with its accurate concentration as opposed to one that could not.

Hubaux and Vos (1970) developed a statistical analysis for determination of MDL for linear calibration curves, and they based their statistical analysis on the fact that the sensitivity of a method can be proportional to its precision, which can be measured based on variance or standard deviation of the prepared samples.

In order to use Hubaux and Vos's method to calculate MDL, at least three different standards (I) should be prepared. However, increasing the number of standards from three to six will result in substantial gain in the sensitivity of the method (Hubaux and Vos 1970). The replicates of each sample (J) can vary starting from one. The number of measurements of each replicate can also vary starting from one (L). The method calculates the MDL by assuming that the measurements from the instrument (in this case UV-Vis spectrophotometer) versus the concentration of each prepared standard forms a linear line with the following equation:

$$
Y=b X+a
$$

Constants $\mathrm{b}$, and a are calculated via equations 2.2 , and 2.3 below:

$$
\begin{gathered}
b=\frac{\sum_{i=1}^{i=n}\left[\left(x_{i}-\bar{x}\right)\left(y_{i}-\bar{y}\right)\right]}{\sum_{i=1}^{i=n}\left(x_{i}-\bar{x}\right)^{2}} \\
a=\bar{y}-b \bar{x}
\end{gathered}
$$

Where $\bar{x}$ and $\bar{y}$ are the average concentration of all of the standards, and their corresponding measurements respectively. In the next step, the standard error for regression is calculated as follows: 


$$
S_{y / x}=\sqrt{\frac{\sum_{i=1}^{I} \sum_{j=1}^{J}\left(\bar{y}_{i j}-\hat{y}_{i}\right)^{2}}{I \times J-2}}
$$

Where $\hat{y}_{i}$ is the calculated value of calibration standard's response calculated from equation 2.1,2.2, and 2.3 , and $\bar{y}_{i j}$ is the average of readings for $\mathrm{J}$ number of replicates.

Using 95\% confidence interval and $n=J \times I$ degrees of freedom, the student's $\mathrm{t}$ distribution value from one-tail distribution shall be extracted from a table as $t_{(0.05, n-2)}$. Using the values calculated from equations 2.2 through 2.4 , critical concentration $\mathrm{x}_{\mathrm{c}}$ (the concentration that can be distinguished from blank), and critical response $\mathrm{y}_{\mathrm{c}}$ (instrument response to the critical concentration) are calculated as follows:

$$
\begin{gathered}
x_{c}=\frac{t_{(0.05, n-2)} \times S_{y / x}}{b} \times \sqrt{\frac{1}{K}+\frac{1}{I \times J}+\frac{\bar{x}^{2}}{J \times \sum_{i=1}^{I}\left(x_{i}-\bar{x}\right)^{2}}} \\
Y_{c}=b X_{c}+a
\end{gathered}
$$

Where $\mathrm{K}$ is the number of replicates for an unknown sample that can vary starting from a value of one. Finally, MDL is to be twice as high as the critical concentration as follows:

$$
M D L=2 X_{c}
$$

The above procedure is implemented in an excel spreadsheet provided via Chemiasoft website (Chemiasoft.com, accessed February 2014). Snapshot of this spreadsheet is provided in Appendix A, under Figure A.1. This spreadsheet readily calculates the MDL, upon providing $\mathrm{I}, \mathrm{J}, \mathrm{K}$, and $\mathrm{L}$ values, along with standard concentrations and their corresponding measurements using UV-Vis spectrophotometer. The detection limit calculated for Zetag8160 in deionized water using this spreadsheet results in a value of $\sim 0.2 \mathrm{mg} / \mathrm{L}$, meaning that this method can be relied upon in accurate detection for samples that contain $0.2 \mathrm{mg} / \mathrm{L}$ of Zetag8160 polymer or higher. One would 
expect a detection limit value between $0.1 \mathrm{mg} / \mathrm{L}$, and $0.5 \mathrm{mg} / \mathrm{L}$ based on the linear fit in Figure 2.4 , as the standard with $0.1 \mathrm{mg} / \mathrm{L}$ of polymer does not fall on the linear line whereas the standard with $0.5 \mathrm{mg} / \mathrm{L}$ of polymer does.

\subsubsection{Detection of SNF475 with UV-Vis Spectroscopy}

After successful results with the Zetag8160 polymer, UV-Vis spectroscopy was tested on a different polymer called SNF475. Absorbance of different concentrations of SNF475 was measured at different wavelengths. As shown below in Figure 2.5, incremental increase in SNF475 polymer concentration exhibited the same trend as Zetag8160 polymer (Figure 2.2). However, the absorbance values for SNF475 polymer were somewhat different than those for Zetag8160. As shown in Figure 2.2, the absorbance of a $40 \mathrm{mg} / \mathrm{L}$ standard of Zetag8160 in deionized water at $\lambda=190 \mathrm{~nm}$ was equal to 1.08 whereas for SNF475 polymer, it is equal to 0.95 (Figure 2.5).

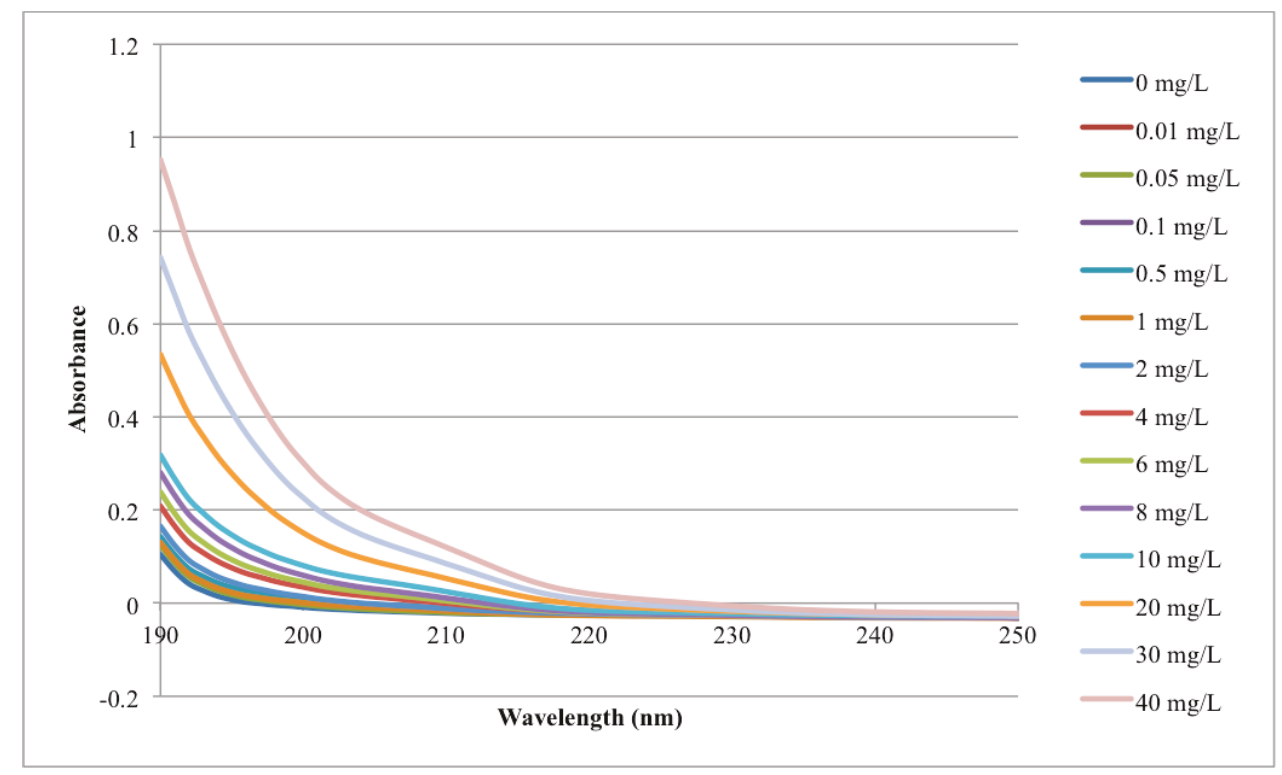

Figure 2.5 UV-Vis spectra of SNF475 polymer (average of three standards)

This difference could be due to the differences between characteristics (charge, molecular weight, structure, etc.) of the polymers. The light absorbance of polymers with 
higher molecular weight would be higher. Another property that could affect the absorbance is the difference between the structures of the polymers. Polymer structure could either be linear, branched, or cross-linked as shown in Figure 2.6, and cross-linked polymers would likely have higher light absorbance due to their higher molecular weight.

Figures 2.7, and 2.8 show the relationship between absorbance and concentration for the SNF475 polymer. Similar to the Zetag8160 polymer in Figure 2.3, the absorbance values of SNF475 at different concentrations adopted a perfect linear trend as demonstrated in Figure 2.7.

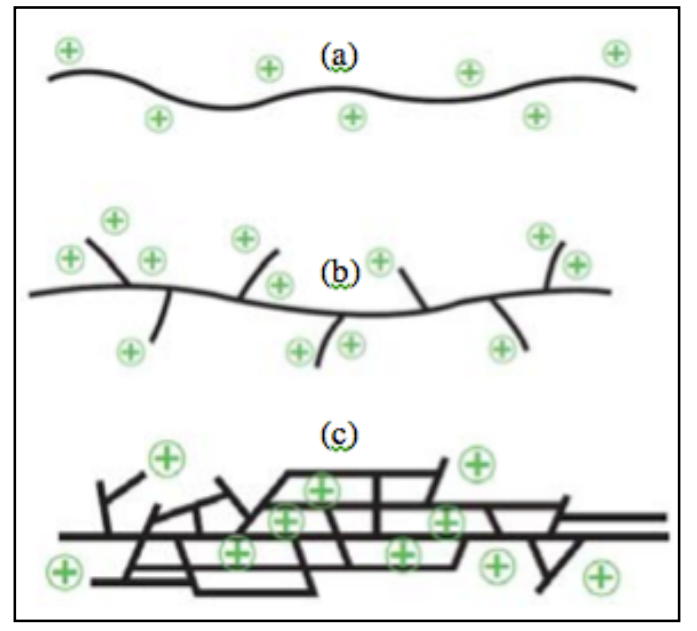

Figure 2.6 (a) Linear polymer, (b) Branched polymer, (c) Cross-linked polymer (De Milieux 2003)

SNF475 could also be detected by UV-Vis spectrophotometer even at very low increases of about $0.01 \mathrm{mg} / \mathrm{L}$ (Figure 2.8). However, since the data were more scattered for SNF475 at low concentrations (Figure 2.8), it was expected to have higher MDL compared to that of Zetag8160. In fact, after calculating the MDL based on Hubaux and Vos's method using Chemiasoft automated excel sheet (Chemiasoft.com, accessed February 2014), the MDL for SNF475 polymer was calculated to be $\sim 0.7 \mathrm{mg} / \mathrm{L}$ which was about $0.5 \mathrm{mg} / \mathrm{L}$ higher than that of Zetag8160. 


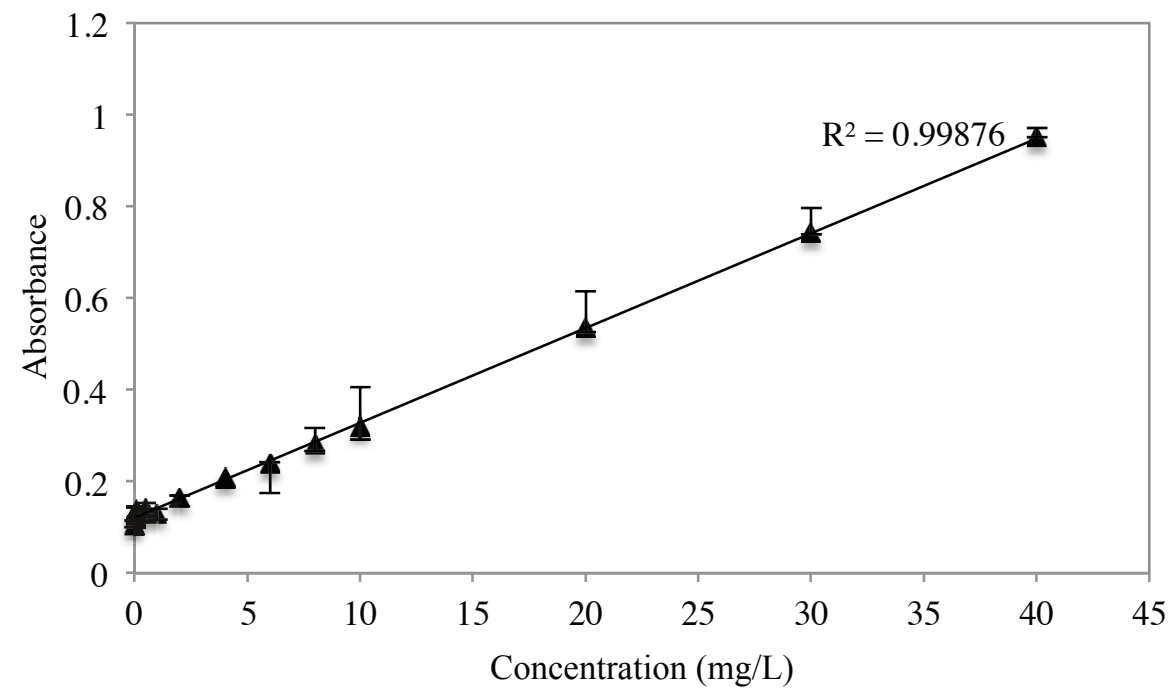

Figure 2.7 Relationship between SNF475 concentration and absorbance at $190 \mathrm{~nm}$ in the high concentration range $(0-40 \mathrm{mg} / \mathrm{L})$

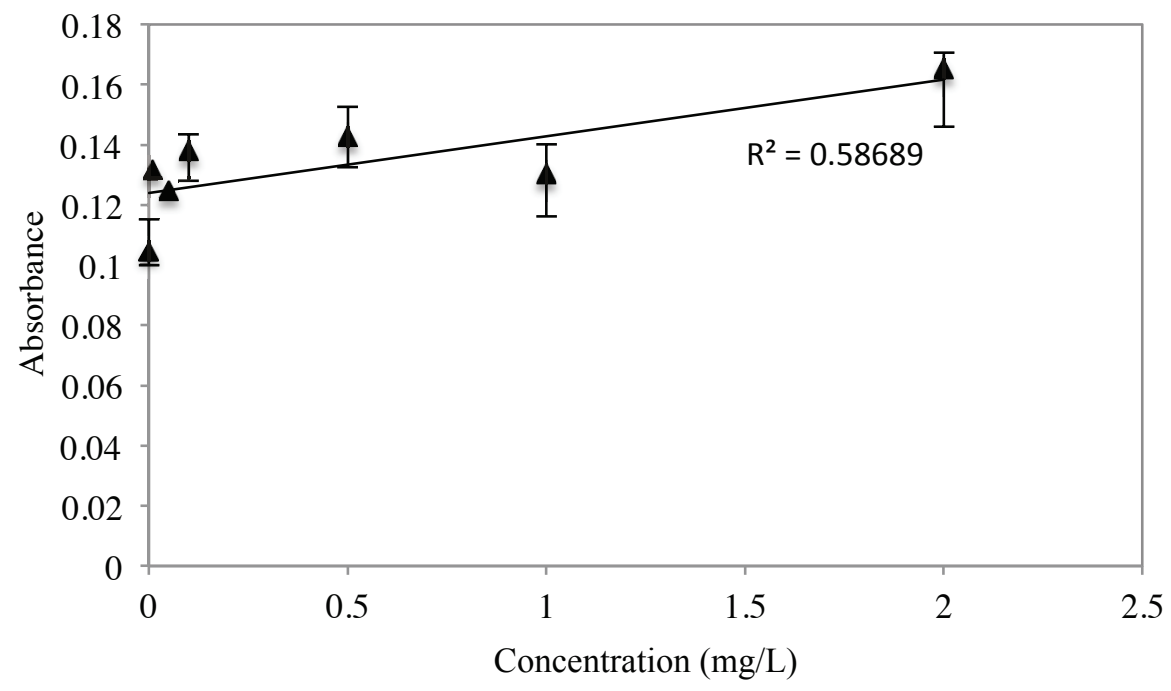

Figure 2.8 Relationship between SNF475 concentration and absorbance at $190 \mathrm{~nm}$ in the low concentration range $(0-2 \mathrm{mg} / \mathrm{L})$

A thorough literature review showed only one other study that used UV-Vis spectroscopy directly to monitor polyacrylamide concentration within fiber suspensions in pulp and paper industry (Howard, Hudson and West 1977). Usually, the solids that 
make up the papers are in dilute aqueous suspension, which is passed through a mesh. This mesh then becomes paper upon removal of water and some other smaller particles, and after further drying and consolidation. Polymers such as polyacrylamide are used in pulp and paper industry in order to increase the retention of finer fibers and pigments on the mesh. Addition of polymers not only thickens the resulting paper, but also reduces the solids in pulp and paper plant effluent. Using UV-Vis spectroscopy, Howard et al. (1977) provided a calibration curve for polyacrylamide in pulp fiber suspension that showed strong absorption in the far UV, peaking at $191.5 \mathrm{~nm}$, and used that to estimate polyacrylamide concentration in pulp fiber suspensions.

Other studies such as Chmilenko et al. (2004), Lu, and Wu (2003), Lu et al. (2003), and Scoggins and Miller (1979, and 1975) used UV-Vis spectroscopy to indirectly measure concentration of polyacrylamide. They derived a complex by reacting polyacrylamide with a chemical, and measured the absorption spectra of the complex via UV-Vis spectroscopy, and later related the absorbance of the complex to the concentration of polyacrylamide. A thorough review of these studies is provided in Chapter 4.

\subsection{Conclusions}

Intensity and absorbance of samples containing different concentrations of Zetag8160 polymer in deionized water were measured using fluorescence and UV-Vis spectroscopy over wavelengths of $200-800 \mathrm{~nm}$, and $190-250 \mathrm{~nm}$ respectively. It was observed that fluorescence intensity of samples were randomly scattered around intensity $=0$ a.u. ( $\mathrm{x}$ axis) and therefore it was concluded that fluorescence spectroscopy could not be used to directly detect and measure the concentration of polyacrylamide polymers in a 
simple manner. The UV-Vis absorbance of Zetag8160 samples, however, exhibited a linear relationship with polymer concentration particularly at $190 \mathrm{~nm}$. In addition SNF475 polymer was tested and a linear relationship between absorbance and concentration was observed again at $190 \mathrm{~nm}$. Likely due to the differences between the two polymers, their corresponding MDLs were found to be different. The MDLs of Zetag8160 and SNF475 were calculated to be $0.2 \mathrm{mg} / \mathrm{L}$ and $0.7 \mathrm{mg} / \mathrm{L}$, respectively. 


\section{Chapter 3: $\quad$ Effects of $\mathbf{p H}$, Salts, and}

\section{Inorganic Conditioners on the Detection}

\section{of Polymers}

\subsection{Introduction}

Anything that can increase or decrease the degree of hydrolysis of polyacrylamide can have impact on its ability to absorb ultraviolet light. Factors such as $\mathrm{pH}$, presence of certain bacteria, as well as increase in the operational temperature of the experiments have shown to cause changes in the degree of hydrolysis of polyacrylamide (Taylor and Nasr-El-Din 1994).

Polyacrylamide polymers $\left[-\mathrm{CH}\left\{\mathrm{C}(=\mathrm{O})-\mathrm{NH}_{2}\right\}-\mathrm{CH}_{2}-\right]_{\mathrm{n}}$ consist of amide $\left(\mathrm{NH}_{2}\right)$ groups. During hydrolysis, amide groups are converted to carboxylic acids $(\mathrm{COOH})$ (Taylor and Nasr-El-Din 1994), and therefore depending on the degree of hydrolysis, a shift in the peak of absorption of polyacrylamide would be expected. This is due to the fact that the amide functionality has an absorption peak at a wavelength of about $185 \mathrm{~nm}$, whereas the acid functionality absorbs at $205 \mathrm{~nm}$ (Muller, Laine and Fenyo 1979), and therefore conversion of amide to carboxylic acid groups will eventually move the peak of absorption of polyacrylamide from $185 \mathrm{~nm}$ towards $205 \mathrm{~nm}$, or, will form a new, but smaller peak at $205 \mathrm{~nm}$, the size of which is dependent on the degree of hydrolysis.

Both acidic and basic solutions can promote hydrolysis of polymers. In both situations, there exists a catalyst. During alkaline hydrolysis, hydrolysis is catalyzed by 
$\mathrm{OH}^{-}$ions in the initial steps, but after increase in the concentration of carboxylic acid groups $(\mathrm{COOH})$, they will start acting as catalysts and result in auto retardation of hydrolysis (Francois and Kheradmand 1988), mainly due to the electrostatic repulsion between the anionic reagent $\left(\mathrm{OH}^{-}\right)$and the polymeric substrate $\left(\mathrm{CH}_{2}-\mathrm{CH}-\mathrm{COO}^{-}\right)$(Truong, Galin and Francois 1986). The reaction for the alkaline hydrolysis of polyacrylamide is shown below (Chmilenko, Korobova and Nazarenko 2004):

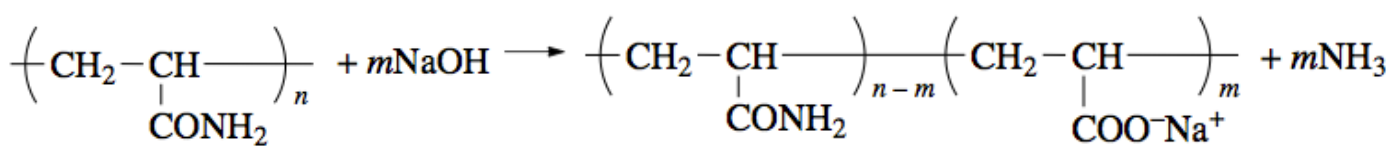

In acidic solutions, however, the carboxylic acid groups act as catalysts from the start of the reaction due to the limited quantity of $\mathrm{OH}^{-}$ions, and consequently produce much slower hydrolysis rates compared to that of alkaline environments. Unfortunately, no detailed chemical reaction as shown for the case of alkaline hydrolysis, was found for the acidic hydrolysis of polyacrylamide in literature because of its impracticality that is caused by the imidization effects of $\mathrm{NH}_{2}$ groups (Chmilenko, Korobova and Nazarenko 2004), which is the formation of aromatic molecules by creation of a new bond between nitrogen and an adjacent carbon atom, bearing only one hydrogen atom, from neighbor acrylamide molecule.

In addition to polyacrylamide hydrolysis that results in modifications in the molecules forming the polymeric chain and therefore leading to appearance of a new peak in polymer spectra representing carboxylic acids, certain material such as salts, as well as some dissolved organic or inorganic constituents have also been known to cause interference in certain polyacrylamide detection techniques. 
Salts on their own do not affect the degree of hydrolysis of polyacrylamide. However, salts especially those of divalent ions such as $\mathrm{CaCl}_{2}$, result in a decrease in the hydrodynamic volume of the polymer, which may affect some analytical methods such as size exclusion chromatography that work based on the size of the polymer molecules (Taylor and Nasr-El-Din 1994). Other properties of polymers that can change by the degree of salinity are the rheological properties such as viscosity, shear thickening, and anti-thixotropic properties. One other interference of salts, especially those of divalent ions would be chelation with partially hydrolyzed polyacrylamide at high concentrations that can result in polymer precipitation, which will lead to false polymer detection (Taylor and Nasr-El-Din 1994).

None of the factors mentioned above would be expected to change the molecular structure and thus absorbance spectra of polymers. However, salts may potentially absorb light in the same UV-Vis range as polyacrylamide and this should be checked for various salts. Indeed, Korshin et al. (1997) showed that salts with chloride ion such as $\mathrm{NaCl}$, and $\mathrm{CaCl}_{2}$ interfere with polyacrylamide spectra at wavelengths lower than $200 \mathrm{~nm}$ due to the existence of chloride ions, which can absorb ultraviolet light at those wavelengths.

Inorganic conditioners (e.g., ferric chloride, ferrous chloride and alum) used at water and wastewater treatment plants may also absorb ultraviolet light and they should be checked for this. UV absorbance of inorganic conditioners in the far UV range has not been studied before. However, the studies of Chow et al. (1999) and Korshin et al. (1997) that used alum to remove natural organic matter in drinking water and river water, respectively, involves UV-Vis spectroscopy. They used UV-Vis spectroscopy to monitor the changes in water quality caused by the addition of alum and removal of organic 
matter. In both of the studies, the authors saw a decrease in the absorption spectra of water samples in the far UV region of 200-245 $\mathrm{nm}$ due to the improved quality of water. Even though the decrease in absorption spectra was associated to the rising quality of water, the effects of alum in the 200 to $245 \mathrm{~nm}$ region should not be neglected.

Due to the effects of $\mathrm{pH}$ and salts on the absorption spectra of polyacrylamide, as well as the suspected effects of alum, the interference of these elements on absorption spectra of polyacrylamide is investigated in this chapter. Furthermore, investigation on the interference from inorganic conditioners is extended to two other most commonly used coagulants of ferrous chloride, and ferric chloride, in addition to alum. It should be noted, however, that no information corresponding to the absorption of ferrous, and ferric chloride in the ultraviolet region was found in literature.

\subsection{Materials and Methods}

\subsubsection{Polymer Stock Solution}

Polymer stock solutions of $500 \mathrm{mg} / \mathrm{L}$ or $0.05 \%$ were prepared as follows: in a plastic weighing dish, $0.5 \mathrm{~g}$ of SNF475 (polyacrylamide, high charge density, ultra-high molecular weight, granular solid form) polymers were weighted using a scale (SI-114, Denver Instruments, Canada), and decanted into a $1 \mathrm{~L}$ glass beaker. About $1 \mathrm{~L}$ of deionized water was also added to the polymer. The mixture was stirred using a jar tester (Phipps and Bird, USA) at $200 \mathrm{rpm}$ for 5 minutes followed by 55 minutes at $125 \mathrm{rpm}$ and was allowed to rest for 60 minutes prior to usage (Gibbons and Ormeci 2013).

\subsection{2 $\mathbf{p H}$}

In seven $50 \mathrm{~mL}$ centrifuge tubes, required volume of SNF475 stock solution as well as required volume of $\mathrm{HCl}(0.1 \mathrm{~N})$ or $\mathrm{NaOH}(0.4 \%)$ were added to deionized water 
such that the final solutions had polymer concentration equal to $5 \mathrm{mg} / \mathrm{L}$ with different $\mathrm{pH}$ values equal to $3,3.5,4,4.5,5,6,7,8,9,9.5,10,10.5$ and 11 .

In seven other centrifuge tubes, required volume of $\mathrm{HCl}(0.1 \mathrm{~N})$ or $\mathrm{NaOH}(0.4 \%)$ were added to deionized water such that the final solutions had $\mathrm{pH}$ values equal to $3,3.5$, $4,4.5,5,6,7,8,9,9.5,10,10.5$ and 11 . The final volume of all samples was $50 \mathrm{~mL}$.

The final $\mathrm{pH}$ of all the solutions was then measured via $\mathrm{pH}$ meter (ORION 5 star, Thermo Scientific, Canada) in order to ensure that the samples had the desired pH values.

The absorbance of the polymer and deionized water samples was then measured via UV-Vis spectrophotometer (Cary 100 Bio UV-Vis Spectrophotometer, Varian Inc./Aligant Technologies, Canada) and recorded over a range of 190-250 nm using Scan software (Cary WinUV, Varian Inc., Canada).

\subsubsection{Salts}

Stock solutions of $500 \mathrm{mg} / \mathrm{L}$ of two salts of sodium chloride $(\mathrm{NaCl})$, and calcium chloride $\left(\mathrm{CaCl}_{2}\right)$ were prepared in deionized water. In 15 centrifuge tubes, required volume of SNF475 stock solution as well as required volume of $\mathrm{NaCl}$ stock solution were added to deionized water such that the final solutions had polymer concentration equal to $5 \mathrm{mg} / \mathrm{L}$ with $\mathrm{NaCl}$ concentration values equal to $0,0.01,0.05,0.1,0.5,1,2,4,6,8,10$, 20, 30, 40, $50 \mathrm{mg} / \mathrm{L}$. The final volume of samples was $50 \mathrm{~mL}$.

The same steps were repeated with $\mathrm{CaCl}_{2}$ salt. The absorbance of the samples was then measured using UV-Vis spectrophotometer over a range of 190-250 nm.

\subsubsection{Alum}

Stock solution of $1000 \mathrm{mg} / \mathrm{L}$ of alum was prepared in deionized water. In 15 centrifuge tubes, required volume of the stock solutions were added to deionized water 
such that the final solutions had concentration values equal to $0,1,2,4,6,8,10,20,30$, $40,50,100,250,500$, and $1000 \mathrm{mg} / \mathrm{L}$. The absorbance of the samples was then measured using UV-Vis spectrophotometer over a range of 190-250 nm.

\subsubsection{Ferric Chloride}

Stock solution of $500 \mathrm{mg} / \mathrm{L}$ of ferric chloride was prepared in deionized water. In 16 centrifuge tubes, required volume of the stock solutions were added to deionized water such that the final solutions had concentration values equal to $0,0.01,0.05,0.1,0.5,1,2$, $4,6,8,10,20,30,40,50$, and $100 \mathrm{mg} / \mathrm{L}$. The absorbance of the samples was then measured using UV-Vis spectrophotometer over a range of 190-250 nm.

\subsubsection{Ferrous Chloride}

Stock solution of $500 \mathrm{mg} / \mathrm{L}$ of ferrous chloride was prepared in deionized water. In 12 centrifuge tubes, required volume of the stock solutions were added such that the final solutions had concentration values equal to $0,0.001,0.005,0.01,0.05,0.5,1,2,4$, 6, 8, and $10 \mathrm{mg} / \mathrm{L}$. Each centrifuge tube was then diluted with deionized water to have a final volume of $50 \mathrm{~mL}$. The absorbance of the solutions was then measured using UV-Vis spectrophotometer over a range of 190-250 nm.

\subsection{Results and Discussion}

\subsubsection{Effects of pH on Polymer Spectra}

Figures 3.1, through 3.7 show the changes in absorbance of $5 \mathrm{mg} / \mathrm{L} \mathrm{SNF475}$ polymer in deionized water, and absorbance of deionized water having the same $\mathrm{pH}$ as the polymer solution, as $\mathrm{pH}$ is changed to $3,5,6,7,8,9$, and 11 respectively. In all of the figures, the absorbance of deionized water without the addition of further acid or base is also provided as a baseline. It should be noted that measuring the $\mathrm{pH}$ of the samples 
prepared in deionized water was very tricky as deionized water lacks ions and makes it hard for the $\mathrm{pH}$ meter to measure the $\mathrm{pH}$ of the solution. Due to this reason the $\mathrm{pH}$ meter was allowed to rest in the solution for 10 to 15 minutes before a value was recorded as the $\mathrm{pH}$.

Based on the explanations provided in the introduction section, a shift in the polymer spectra is expected due to the conversion of amide groups to carboxylic acid groups since acidic and alkaline environments promote the hydrolysis of polyacrylamide, and upon hydrolysis, the peak of the amide groups that is expected to be on $185 \mathrm{~nm}$, will shift towards the peak for carboxylic acid groups on $205 \mathrm{~nm}$. As seen in Figures 3.1 through 3.6, there is a small peak right at $205 \mathrm{~nm}$ in polymer containing samples indicating the formation of carboxylic acid groups. Figure 3.1 at $\mathrm{pH}=3$ shows higher absorbance value at $190 \mathrm{~nm}$ compared to Figures 3.2 through 3.6 due to the higher hydrolysis occurring at $\mathrm{pH} 3$ compared to $\mathrm{pH} 5,6,7,8$ and 9. However, by comparing Figure 3.1 , to 3.7 for $\mathrm{pH}=3$ and 11 respectively, Figure 3.1 shows lower absorbance values at $190 \mathrm{~nm}$ compared to that of Figure 3.7. This is a direct result of higher hydrolysis rates in strong alkaline solutions due to the role of $\mathrm{OH}^{-}$ions as catalyst in comparison to acidic conditions.

The increase in the absorbance of deionized water after the addition of $\mathrm{NaOH}$, and $\mathrm{HCl}$ in Figures 3.1 through 3.7 is due to the addition of ions in deionized water. Deionized water lacks ions or molecules that can absorb ultraviolet light but as soon as it is introduced to $\mathrm{HCl}$, and $\mathrm{NaOH}$, free ions of $\mathrm{Cl}^{-}$(Korshin, $\mathrm{Li}$ and Benjamin 1997) and, $\mathrm{OH}^{-}$(Zuman and Szafranski 1976) absorb ultraviolet light and therefore result in an increase in the absorbance of water. 
In Figure 3.7 for $\mathrm{pH}$ of 11, the spectra of polymer and water almost adopt the same trend and values. Based on the study done by Zuman and Szafranski (1976), on the spectra of solutions of sodium hydroxide in distilled water, an increase of the absorbance in the region of $200-220 \mathrm{~nm}$ is observed as concentration of $\mathrm{NaOH}$ in the solution is increased. Since their spectrophotometer did not capture absorbance at wavelengths lower than $200 \mathrm{~nm}$, by assuming a symmetrical curve, they concluded that the peak of absorption would be somewhere around 190-200 nm. This corresponds to the wavelength where the absorbance of polyacrylamide is also very high.

Figures 3.1 , and 3.7 for $\mathrm{pH}=3$, and 11 respectively, show the lowest difference between the absorbance of polymer and deionized water samples in spite of an increase in absorbance values. In other words, the more the samples deviate from neutral $\mathrm{pH}$ values, the less will be the difference between the absorbance of polymer and deionized water and thus detection of polymer will become more difficult. What these results suggest is that for the extreme cases of $\mathrm{pH}=3$ and 11 , the absorbance method would not be successful in detecting polymer concentrations.

Moving to the neutral $\mathrm{pH}$ values (Figures 3.2 through 3.6 for $\mathrm{pH}$ values equal to $5,6,7,8$, and 9), there is still significant difference between the absorbance of polymer and deionized water indicating the effectiveness of absorbance method at those $\mathrm{pH}$ values. There are however variations between the absorbance of polymer and deionized water at different $\mathrm{pH}$ values with no trend, and they seem to be randomly changing from $\sim 0.15$ to $\sim 0.2$ for deionized water and from $\sim 0.25$ to $\sim 0.3$ for polymer.

Figure 3.8 summarizes the absorbance of polymer and deionized water samples at $190 \mathrm{~nm}$ under different $\mathrm{pH}$ values. The trend named "baseline" is the absorbance of 
deionized water with no added acid or base. The sharp increases in absorbance at $\mathrm{pH}$ values of 3 and 11, and the plateau of the absorbance values associated to $\mathrm{pH}$ values ranging from 5 to 9 both for polymer and deionized water can be easily seen in Figure 3.8. Figure 3.9, which is a second replicate of Figure 3.8, also shows the same trend, except the greater difference between polymer and deionized water solutions at nonextreme $\mathrm{pH}$ conditions is more evident in this figure. The absorbance spectra of the samples corresponding to Figure 3.9 are shown in Appendix B, under Figures B.1 through B.7. The reason as to why Figures 3.8 and 3.9 are different is most probably due to the duration of the experiments. Cationic polymers have been known to lose their charge as they age, and this might affect the results. The SNF475 polymer tended to lose its charge in a matter of 3 to 4 hours, which was much faster than other polymers used in this study and therefore preparation of any sample using the old stock solution could affect results.

The absorbance spectra of $5 \mathrm{mg} / \mathrm{L}$ solution of SNF475 as well as deionized water, at a $\mathrm{pH}$ of $3.5,4,4.5,9.5,10,10.5$ are shown in Figures 3.10 through 3.15 , in order to monitor the effects of $\mathrm{pH}$ at the extreme ends. As seen in these figures, the more the $\mathrm{pH}$ of the samples deviate from neutrality, the less will be the difference between the absorbance of SNF475 samples and deionized water having the same $\mathrm{pH}$. Gradual increase in absorbance (at $\lambda=190 \mathrm{~nm}$ ) of both deionized water and polymer solutions as they become more acidic and basic are shown in Figure 3.16.

Since $\mathrm{pH}$ of water and wastewater samples are typically around 6-7, the results indicate that the method can be successfully used for treatment applications. 


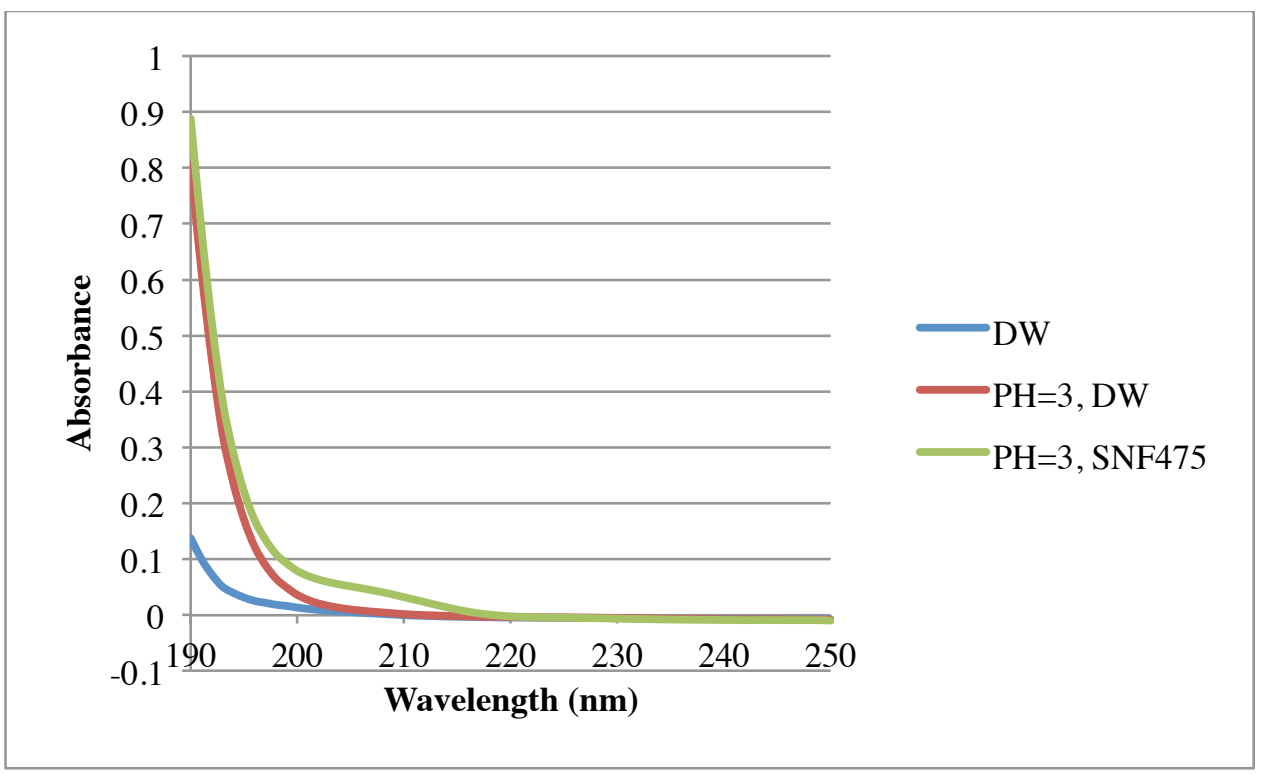

Figure 3.1 Effects of $\mathrm{pH}=3$ on $\mathrm{DW}$, and $5 \mathrm{mg} / \mathrm{L}$ solution of $\mathrm{SNF} 475$ in $\mathrm{DW}$ (average of three measurements)

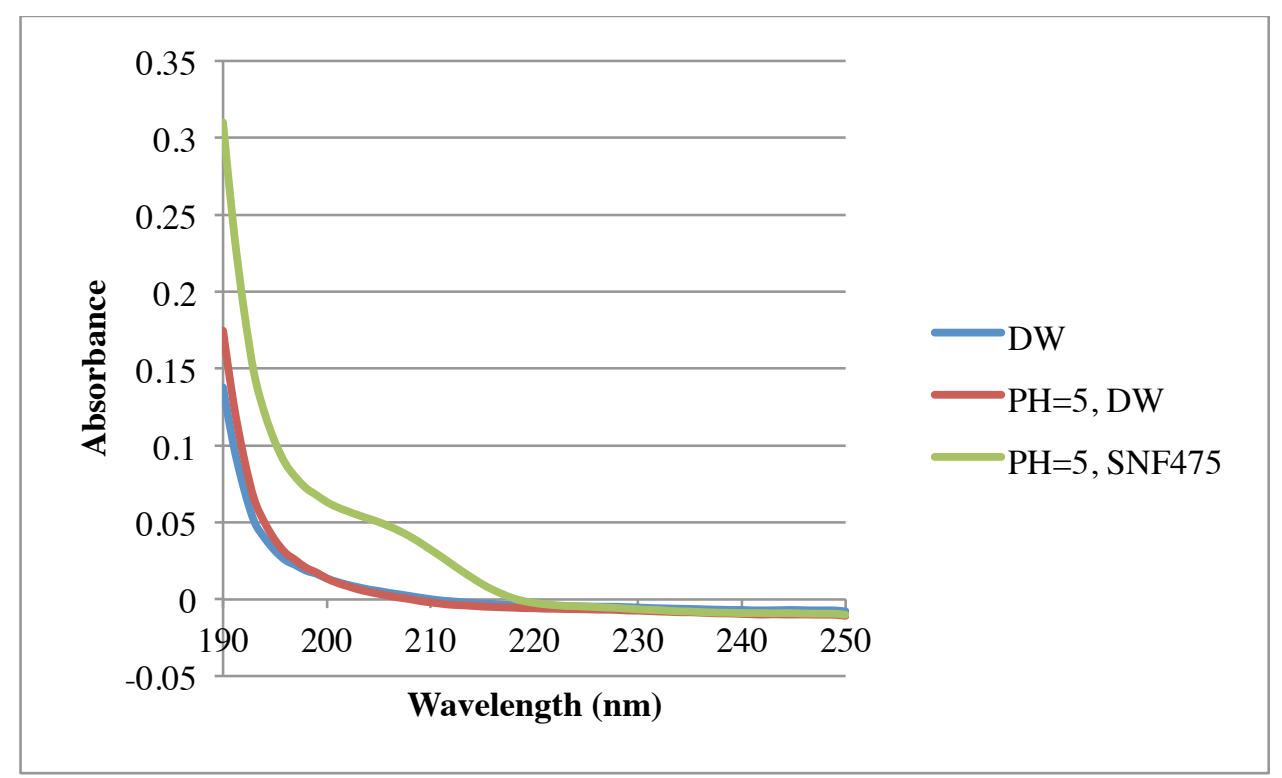

Figure 3.2 Effects of $\mathrm{pH}=5$ on DW, and $5 \mathrm{mg} / \mathrm{L}$ solution of $\mathrm{SNF} 475$ in DW (average of three measurements) 


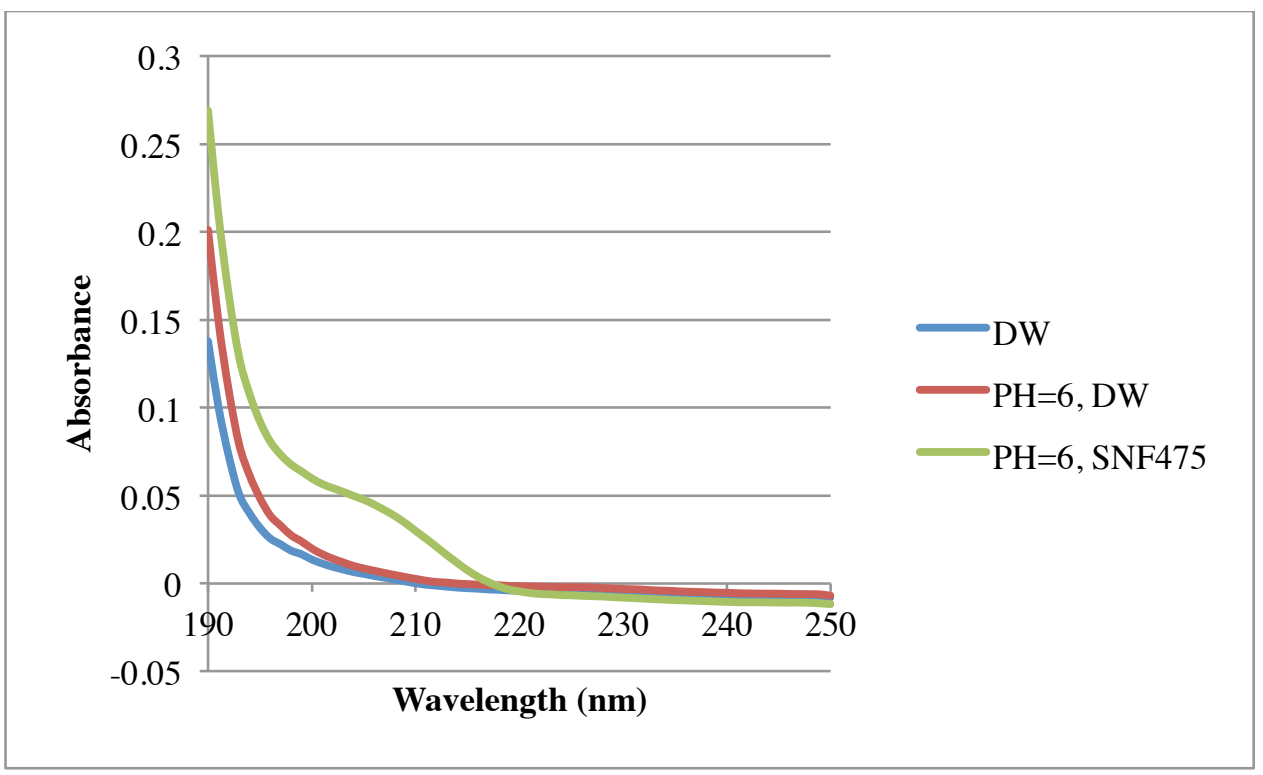

Figure 3.3 Effects of $\mathrm{pH}=6$ on DW, and $5 \mathrm{mg} / \mathrm{L}$ solution of SNF475 in DW (average of three measurements)

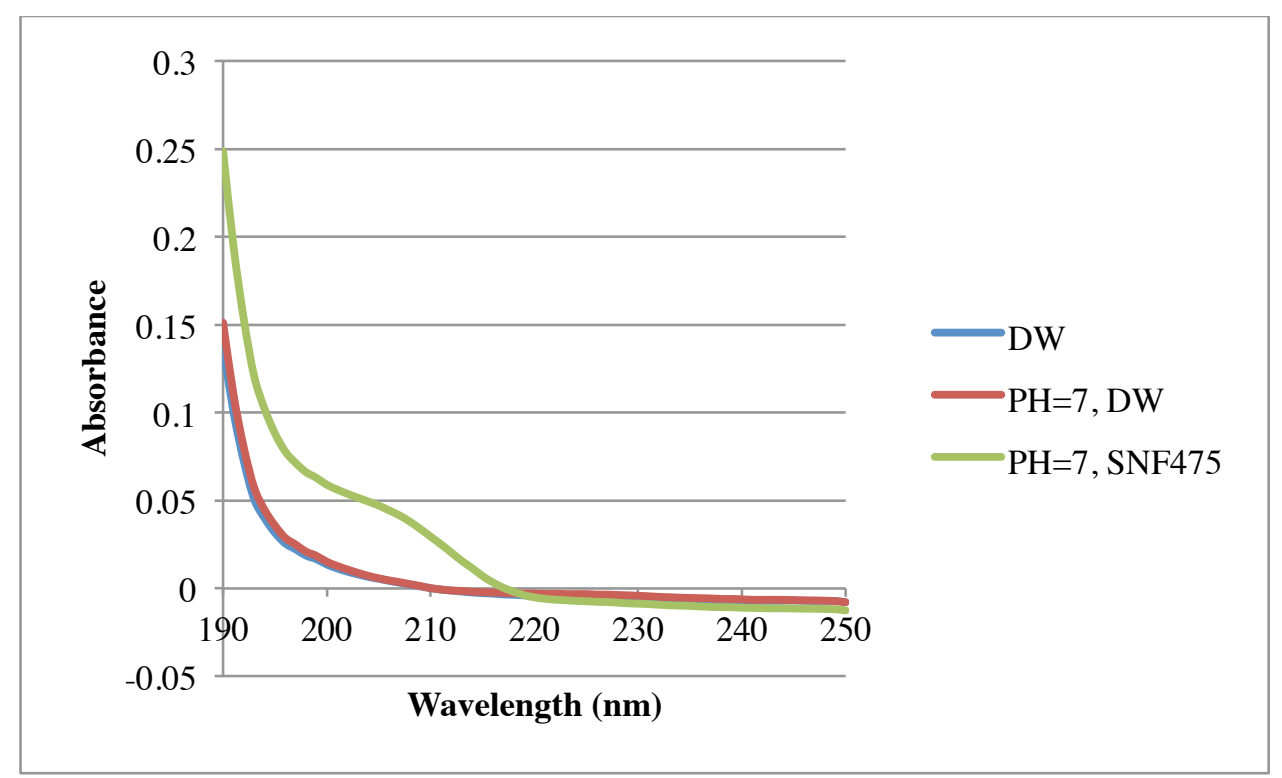

Figure 3.4 Effects of $\mathrm{pH}=7$ on DW, and $5 \mathrm{mg} / \mathrm{L}$ solution of $\mathrm{SNF} 475 \mathrm{in} \mathrm{DW}$ (average of three measurements) 


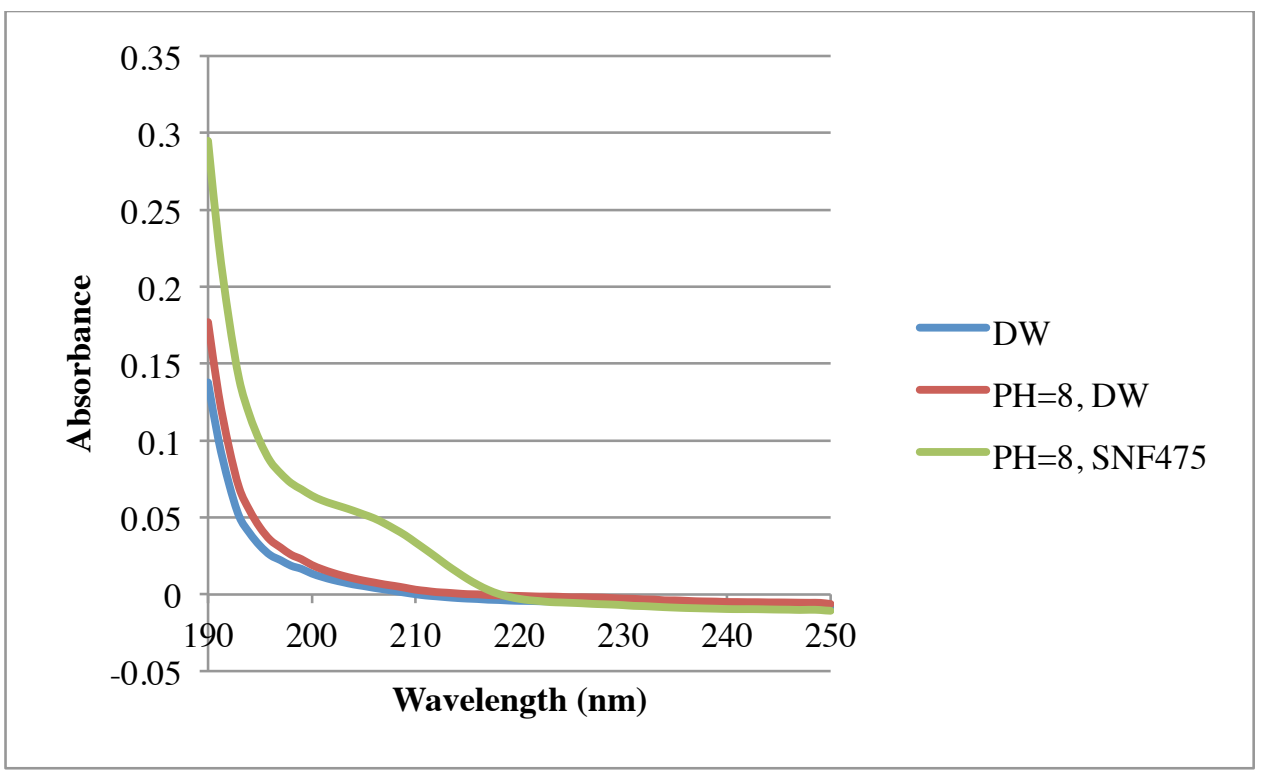

Figure 3.5 Effects of $\mathrm{pH}=8$ on DW, and $5 \mathrm{mg} / \mathrm{L}$ solution of SNF475 in DW (average of three measurements)

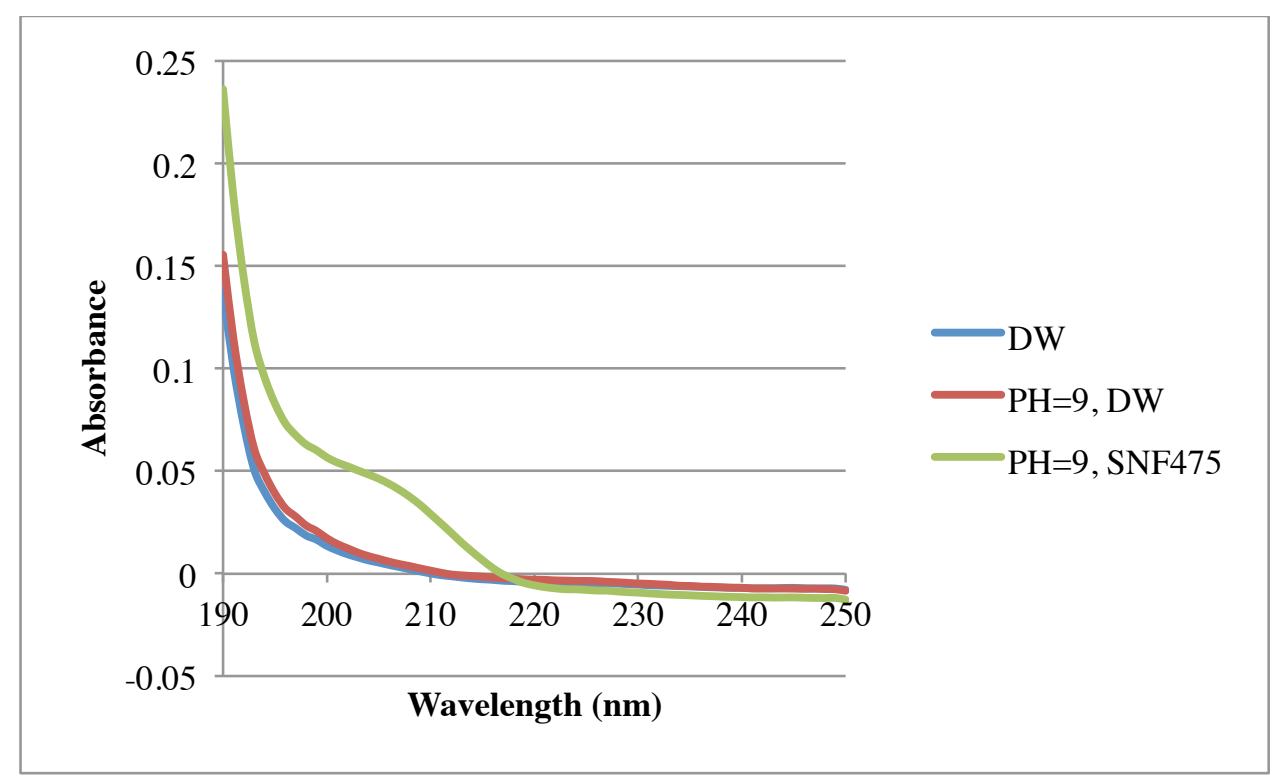

Figure 3.6 Effects of $\mathrm{pH}=9$ on DW, and $5 \mathrm{mg} / \mathrm{L}$ solution of SNF475 in DW (average of three measurements) 


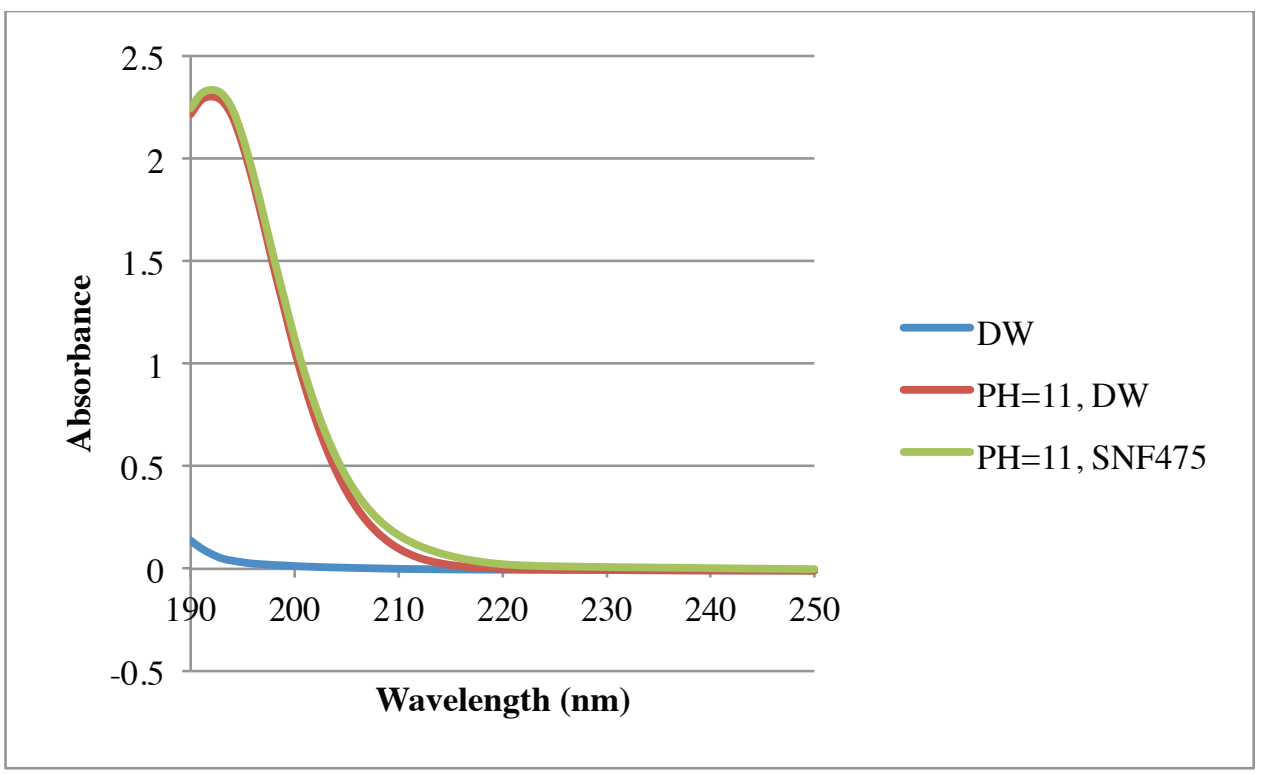

Figure 3.7 Effects of $\mathrm{pH}=11$ on $\mathrm{DW}$, and $5 \mathrm{mg} / \mathrm{L}$ solution of $\mathrm{SNF} 475 \mathrm{in} \mathrm{DW}$ (average of three measurements)

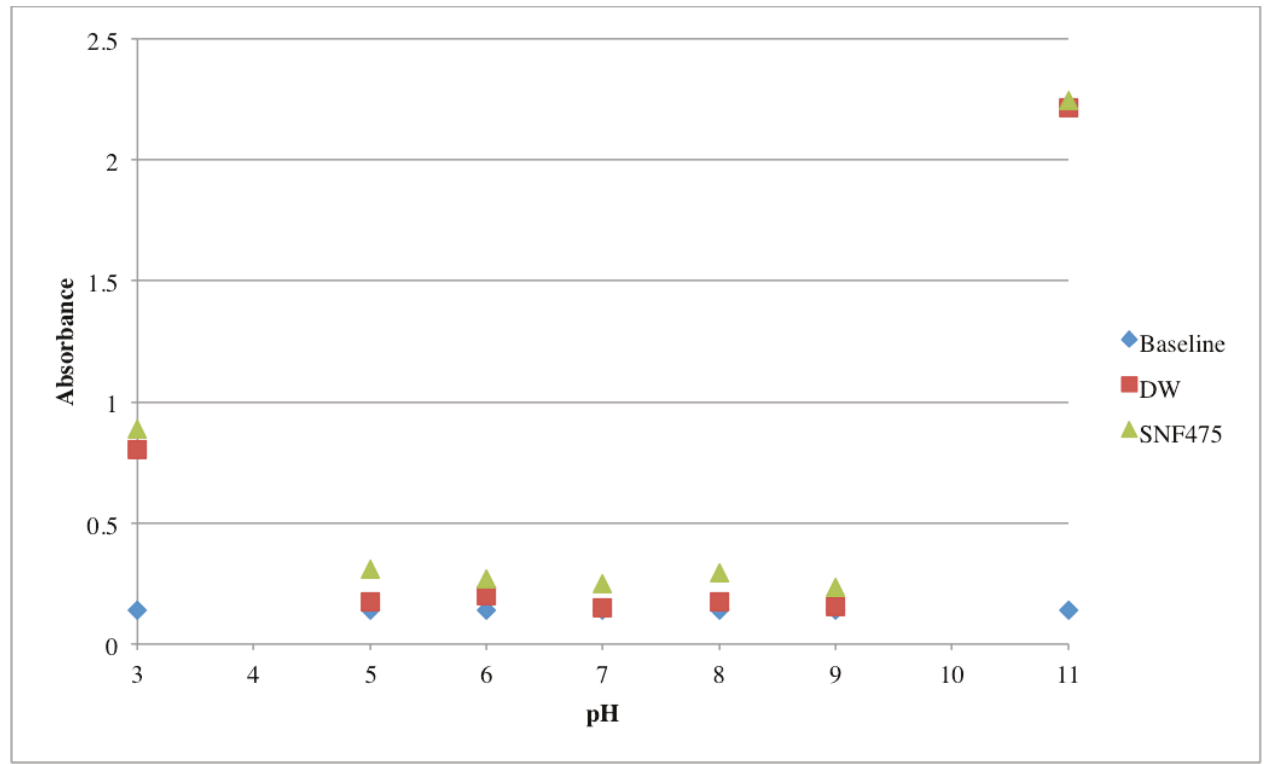

Figure 3.8 Effects of $\mathrm{pH}$ on DW, and $5 \mathrm{mg} / \mathrm{L}$ solution of SNF475 in DW at $\lambda=190 \mathrm{~nm}$ (for replicate 1 - average of three measurements) 


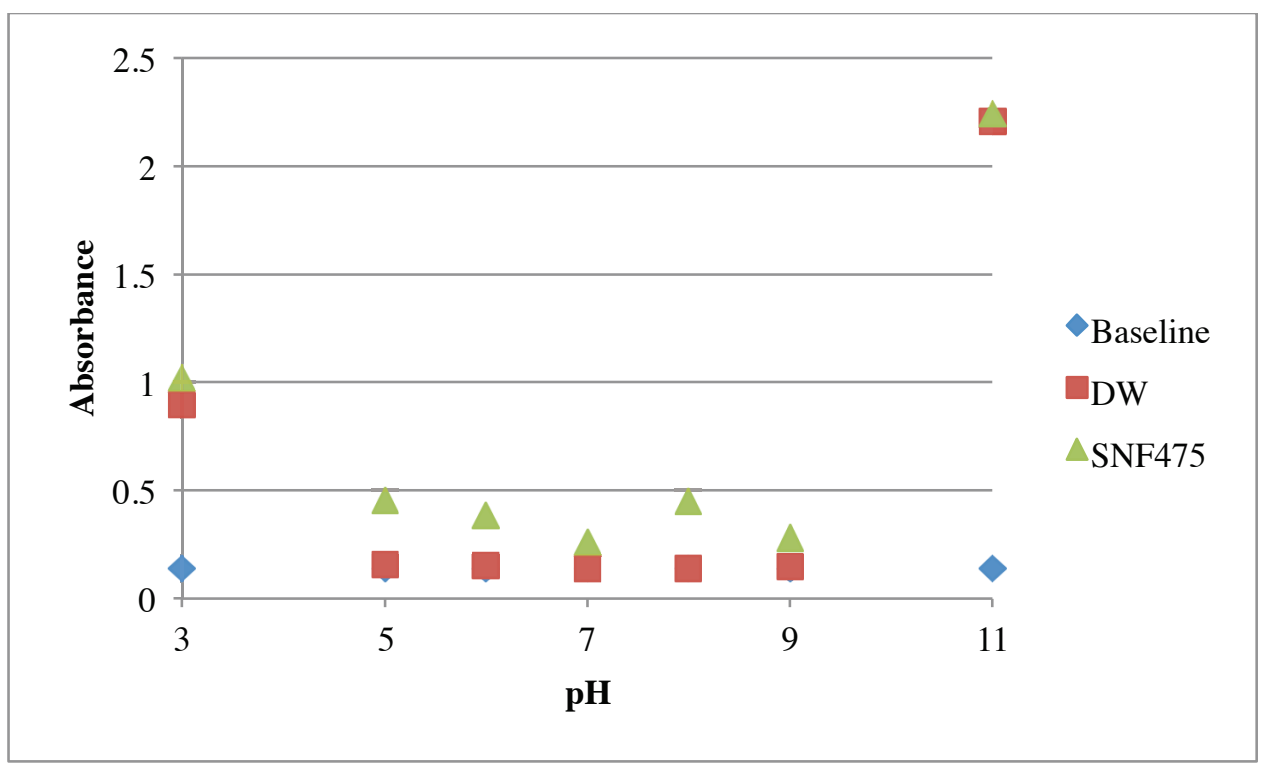

Figure 3.9 Effects of $\mathrm{pH}$ on DW, and $5 \mathrm{mg} / \mathrm{L}$ solution of SNF475 in DW at $\lambda=190 \mathrm{~nm}$ (for replicate 2 - average of three measurements)

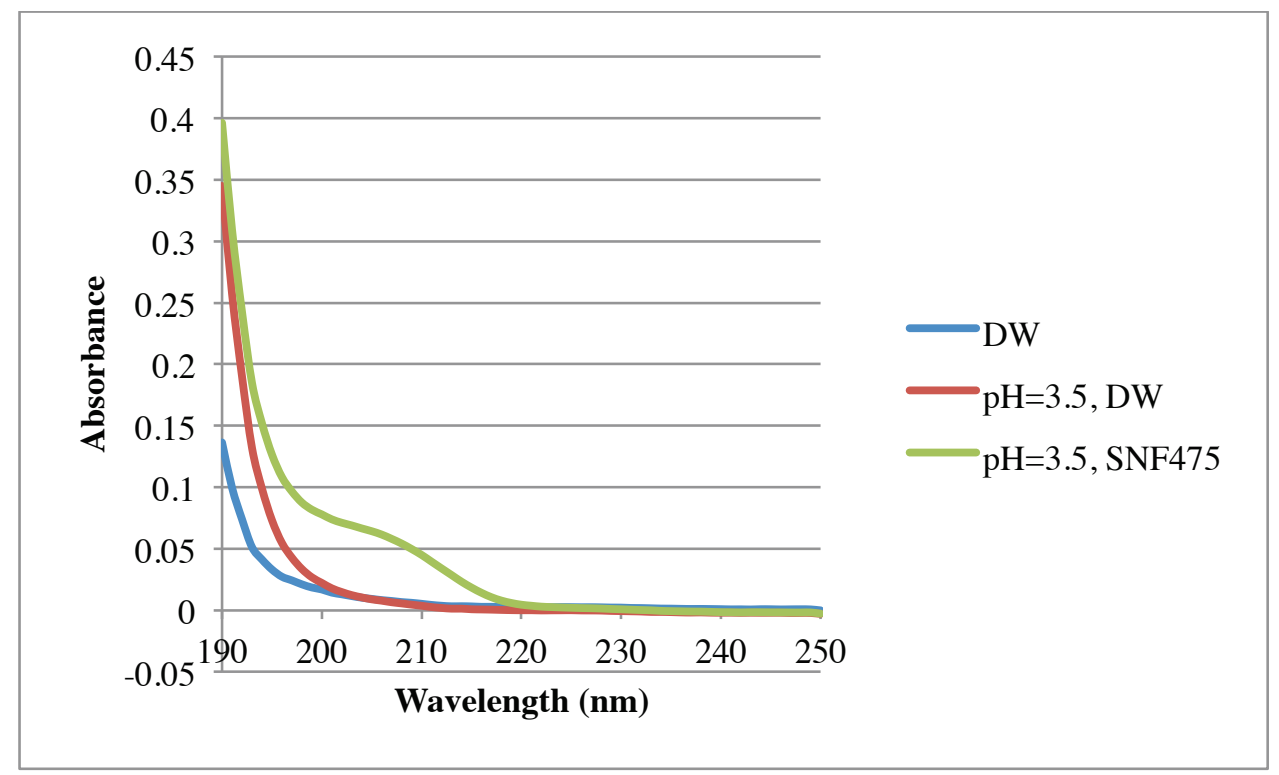

Figure 3.10 Effects of $\mathrm{pH}=3.5 \mathrm{on} \mathrm{DW}$, and $5 \mathrm{mg} / \mathrm{L}$ solution of SNF475 in DW (average of three measurements) 


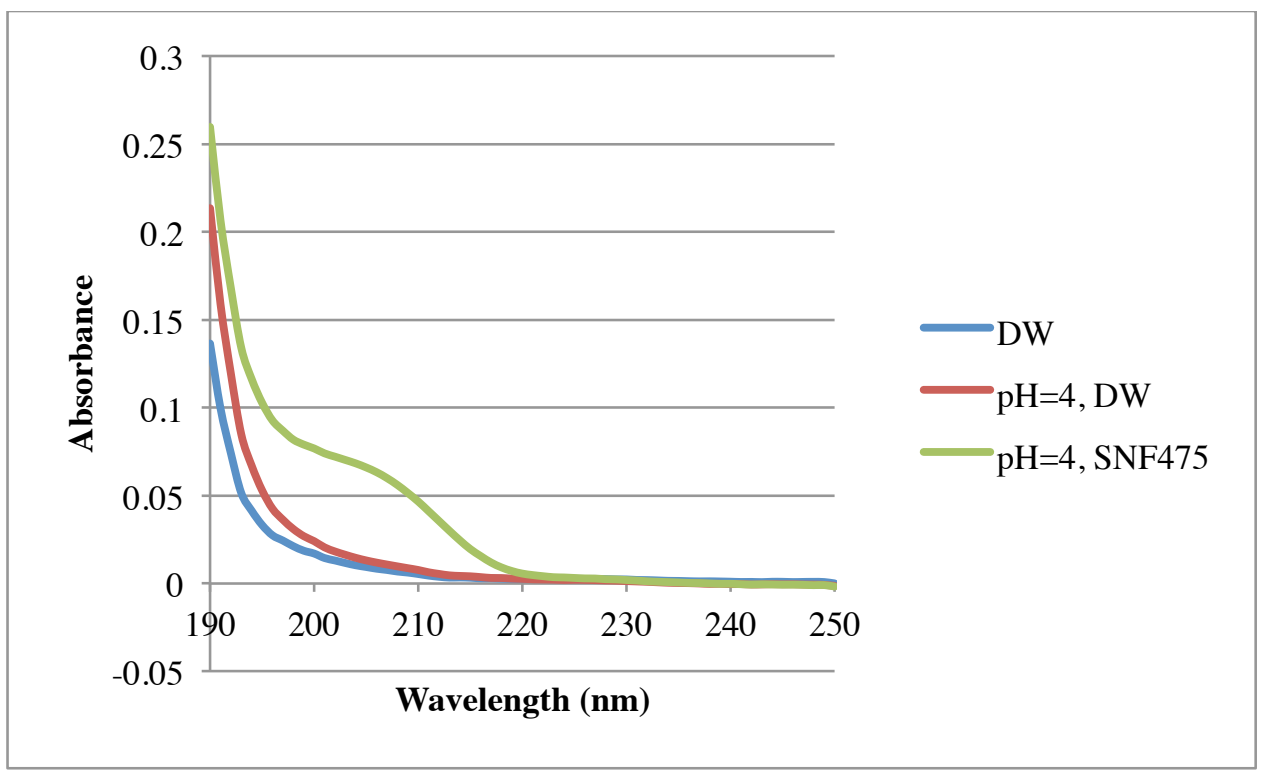

Figure 3.11 Effects of $\mathrm{pH}=4$ on DW, and $5 \mathrm{mg} / \mathrm{L}$ solution of SNF475 in DW (average of three measurements)

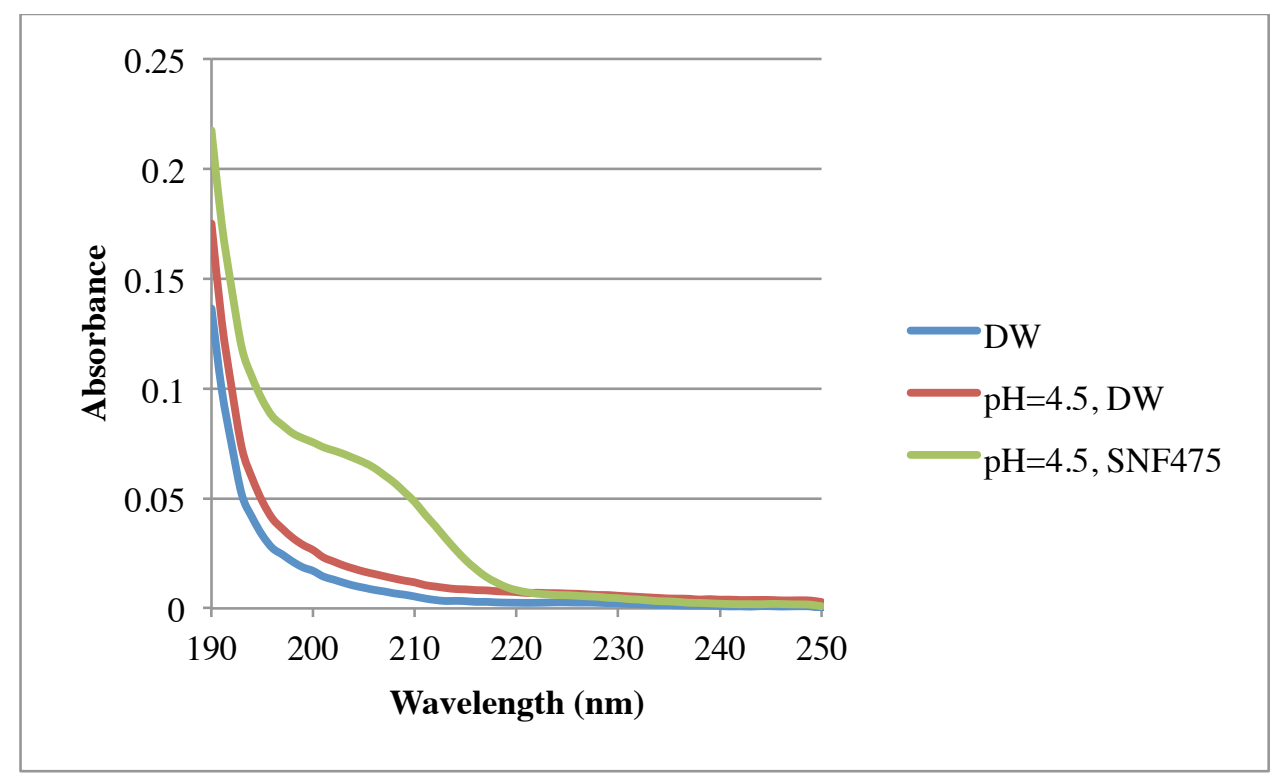

Figure 3.12 Effects of $\mathrm{pH}=4.5 \mathrm{on} \mathrm{DW}$, and $5 \mathrm{mg} / \mathrm{L}$ solution of $\mathrm{SNF} 475$ in $\mathrm{DW}$ (average of three measurements) 


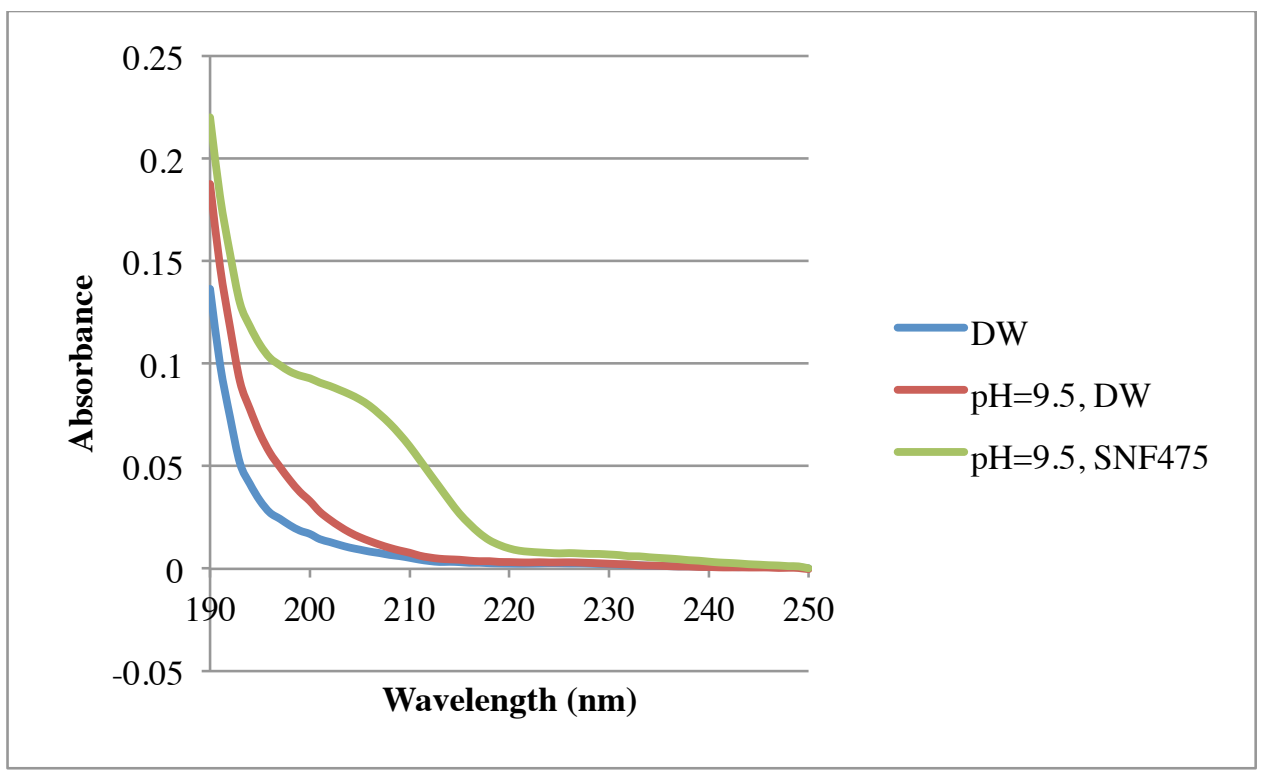

Figure 3.13 Effects of $\mathrm{pH}=9.5 \mathrm{on} \mathrm{DW}$, and $5 \mathrm{mg} / \mathrm{L}$ solution of SNF475 in DW (average of three measurements)

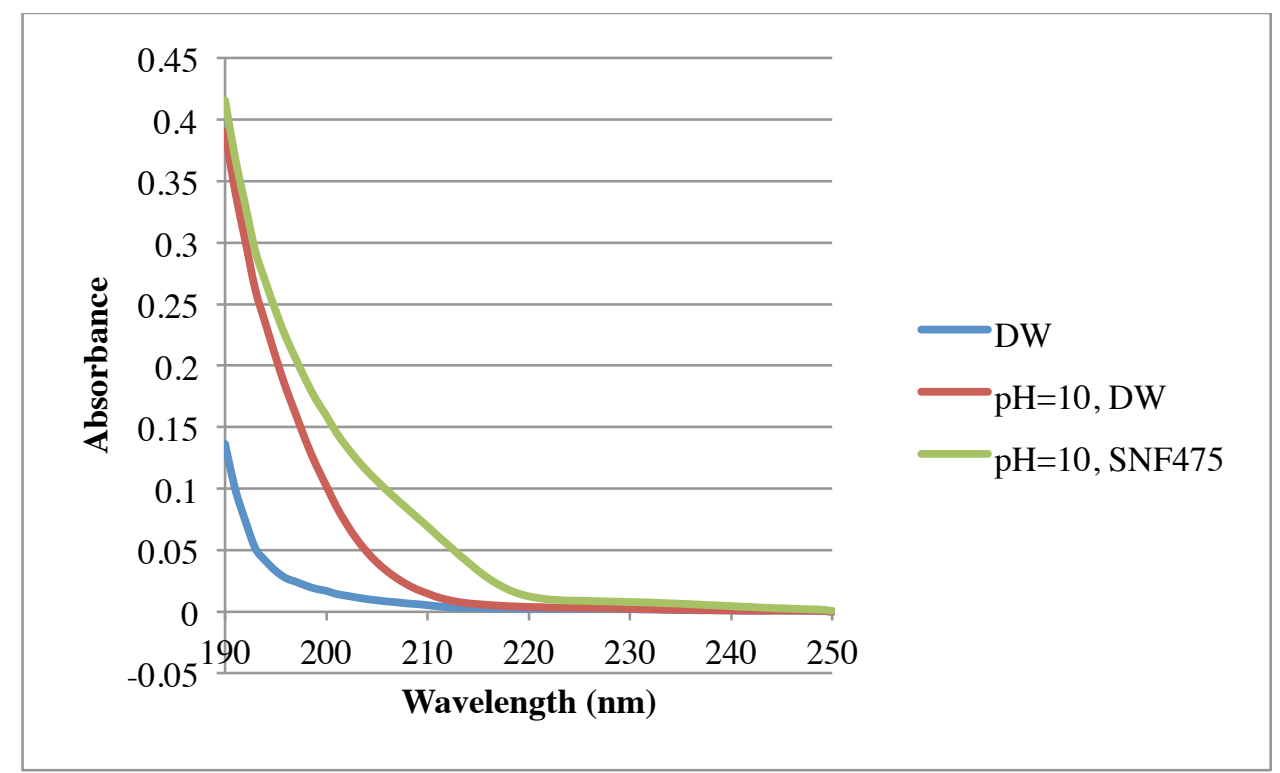

Figure 3.14 Effects of $\mathrm{pH}=10$ on DW, and $5 \mathrm{mg} / \mathrm{L}$ solution of SNF475 in DW (average of three measurements) 


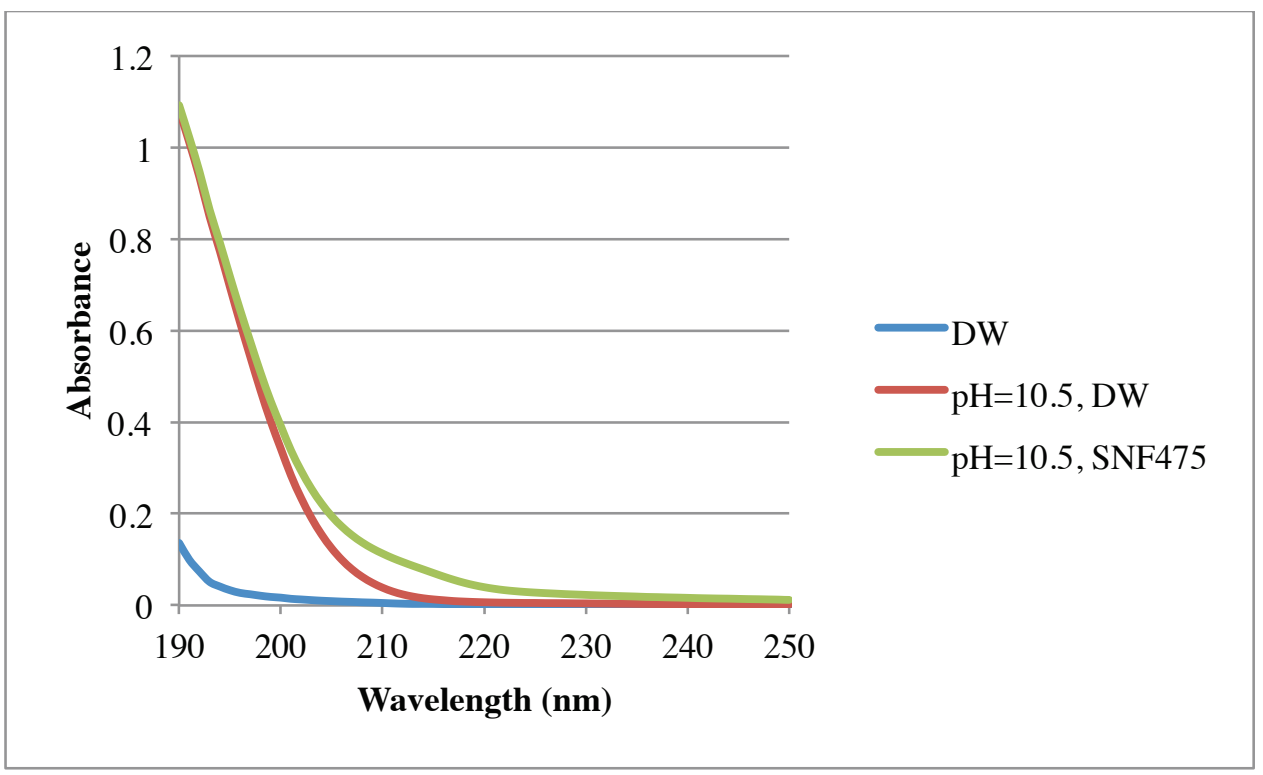

Figure 3.15 Effects of $\mathrm{pH}=10.5 \mathrm{on} \mathrm{DW}$, and $5 \mathrm{mg} / \mathrm{L}$ solution of SNF475 in DW (average of three measurements)

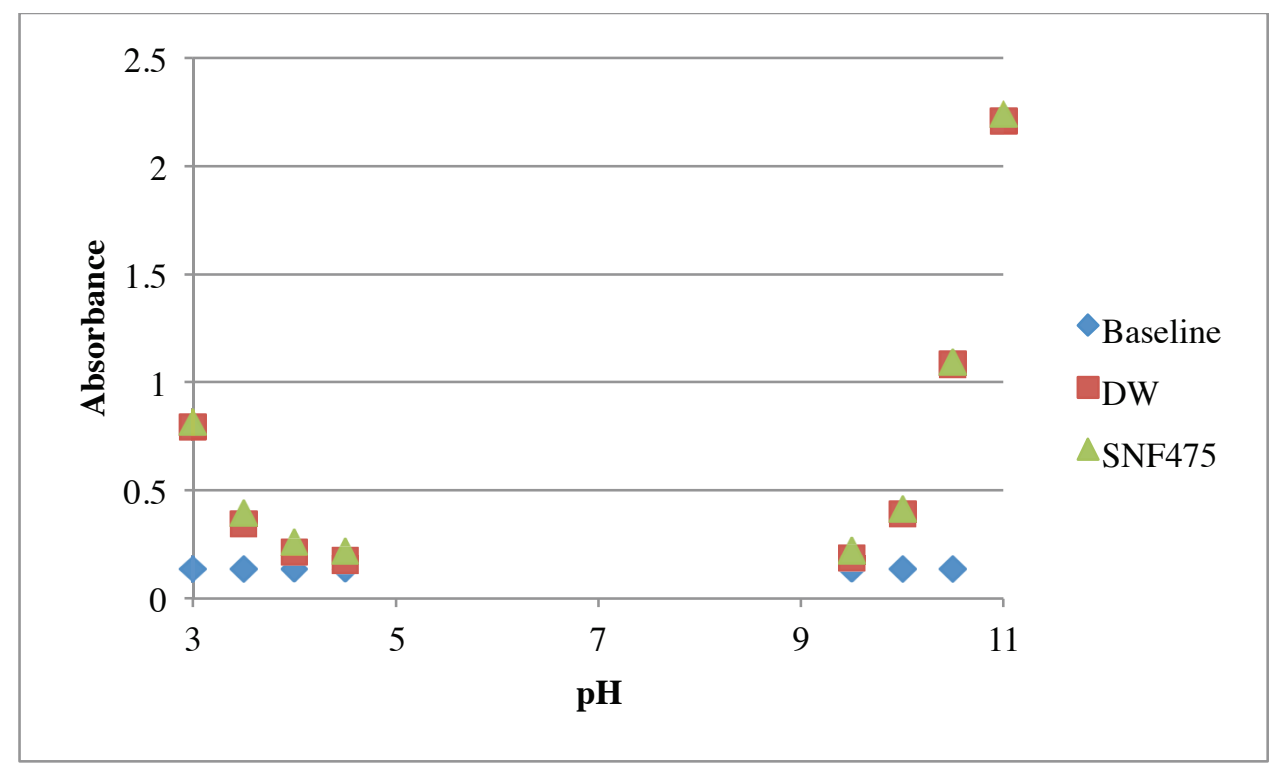

Figure 3.16 Effects of $\mathrm{pH}$ on DW, and $5 \mathrm{mg} / \mathrm{L}$ solution of $\mathrm{SNF} 475 \mathrm{in} \mathrm{DW}$ at $\lambda=190 \mathrm{~nm}$ at extreme pH conditions (average of three measurements) 


\subsubsection{Effects of Salts on Polymer Spectra}

The absorbance values over the range of $190-250 \mathrm{~nm}$ for the solutions of $5 \mathrm{mg} / \mathrm{L}$ SNF475 polymer in deionized water with different $\mathrm{NaCl}$ concentrations are plotted in Figure 3.17 with the specific absorbance values at $190 \mathrm{~nm}$ plotted versus $\mathrm{NaCl}$ concentration in Figure 3.18. The difference between absorbance of different doses would be maximum at $\lambda=190 \mathrm{~nm}$, which makes observation of changes easier compared to other wavelengths. The same plots for $\mathrm{CaCl}_{2}$ are provided in Figures 3.19, and 3.20 respectively.

As reported by Korshin et al. (1997), $\mathrm{Cl}^{-}$ions likely started absorbing ultraviolet light at wavelengths lower than $200 \mathrm{~nm}$ for $\mathrm{NaCl}$ and $\mathrm{CaCl}_{2}$ salts. There is also some ultraviolet light being absorbed from 200-215 nm (Figures 3.17 and 3.19). However, since the absorbance curves associated with different salt concentrations for $200<\lambda<215$ were very close to one another, the absorbance was likely mainly caused by the $5 \mathrm{mg} / \mathrm{L}$ SNF475 polymer that existed in the solutions along with the salts.

No information was found in literature on the hydrolysis of polyacrylamide in the presence of salts; however, the small peaks formed at $\lambda=205 \mathrm{~nm}$ on Figures 3.17 , and 3.19 for $\mathrm{NaCl}$, and $\mathrm{CaCl}_{2}$ were likely caused by the carboxylic acids that were created from hydrolysis of amide groups.

Once the absorbance values were plotted against concentration of $\mathrm{NaCl}$, and $\mathrm{CaCl}_{2}$ in Figures 3.18, and 3.20 at $\lambda=190 \mathrm{~nm}$, it was seen that incremental addition of salts to polymer solutions followed a linear increase in the absorbance. Interestingly, the slope and intercept of the regression lines were very similar for both salts. The slope was 0.0109 and 0.0105 , and the intercept was 0.2529 and 0.2577 for $\mathrm{NaCl}$ and $\mathrm{CaCl}_{2}$, 
respectively. Considering the absorbance of the $\mathrm{Cl}^{-}$ions in the solution, $\mathrm{CaCl}_{2}$ was expected to have a regression line twice as steep as that of $\mathrm{NaCl}$, but it was not the case here. This was because for the same concentration of $\mathrm{NaCl}$, and $\mathrm{CaCl}_{2}$, about the same moles of $\mathrm{Cl}^{-}$was introduced into the solution as follows:

$$
\begin{aligned}
& \frac{1 \frac{\mathrm{mg} \mathrm{NaCl}}{\mathrm{L}}}{58.44 \frac{\mathrm{mg} \mathrm{NaCl}}{\mathrm{mmol} \mathrm{NaCl}}} * 1 \frac{\mathrm{mmol} \mathrm{Cl}}{1 \mathrm{mmol} \mathrm{NaCl}}=0.017 \frac{\mathrm{mmol} \mathrm{Cl}^{-}}{\mathrm{L}} \\
& \frac{1 \frac{\mathrm{mg} \mathrm{CaCl}}{2}}{110.98 \frac{\mathrm{mg} \mathrm{CaCl}}{\mathrm{mmol} \mathrm{CaCl}_{2}}} * 2 \frac{\mathrm{mmol} \mathrm{Cl}^{-}}{1 \mathrm{mmol} \mathrm{CaCl}_{2}}=0.018 \frac{\mathrm{mmol} \mathrm{Cl}^{-}}{\mathrm{L}}
\end{aligned}
$$

The same number of moles of $\mathrm{Cl}^{-}$has the same number of ions based on Avogadro's number, and that was why the same linear trend was achieved as salt concentration was increased in a given solution of polymer.

These results show that if a sample is suspected of containing salts, still the concentration of polymer can be detected within it. However, this needs adjustments in the calibration curve as the baseline is now a sample containing salts and not just pure deionized water. 


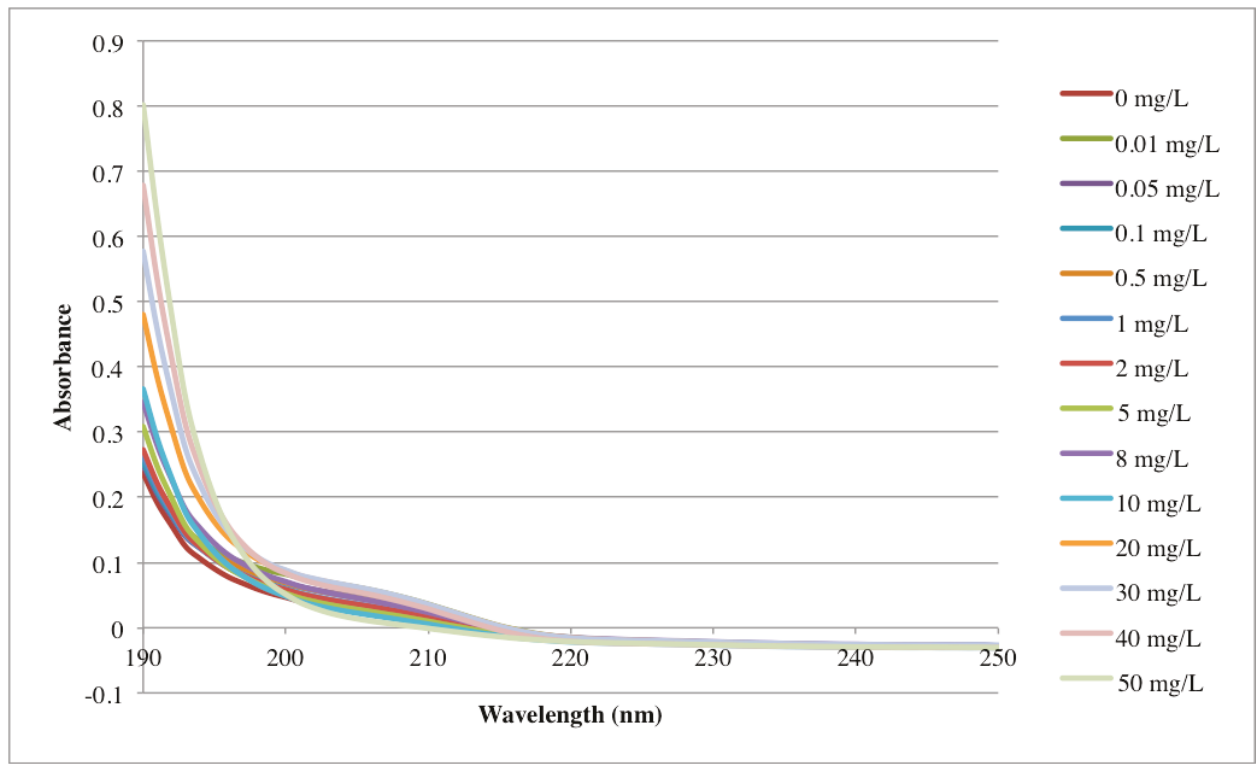

Figure 3.17 Absorbance spectra of $\mathrm{NaCl}$ in $5 \mathrm{mg} / \mathrm{L}$ solution of SNF475 (average of three measurements)

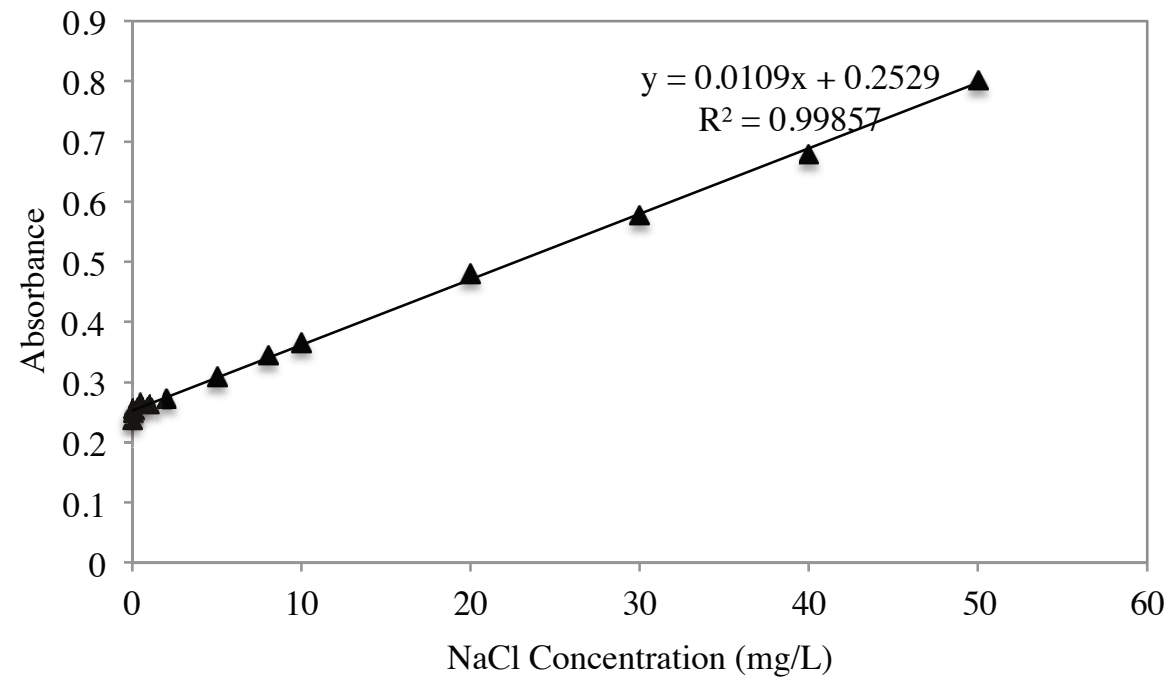

Figure 3.18 Relationship between $\mathrm{NaCl}$ concentration and absorbance in $5 \mathrm{mg} / \mathrm{L}$ solution of $\mathrm{SNF} 475$ at $\lambda=190 \mathrm{~nm}$ (average of three measurements) 


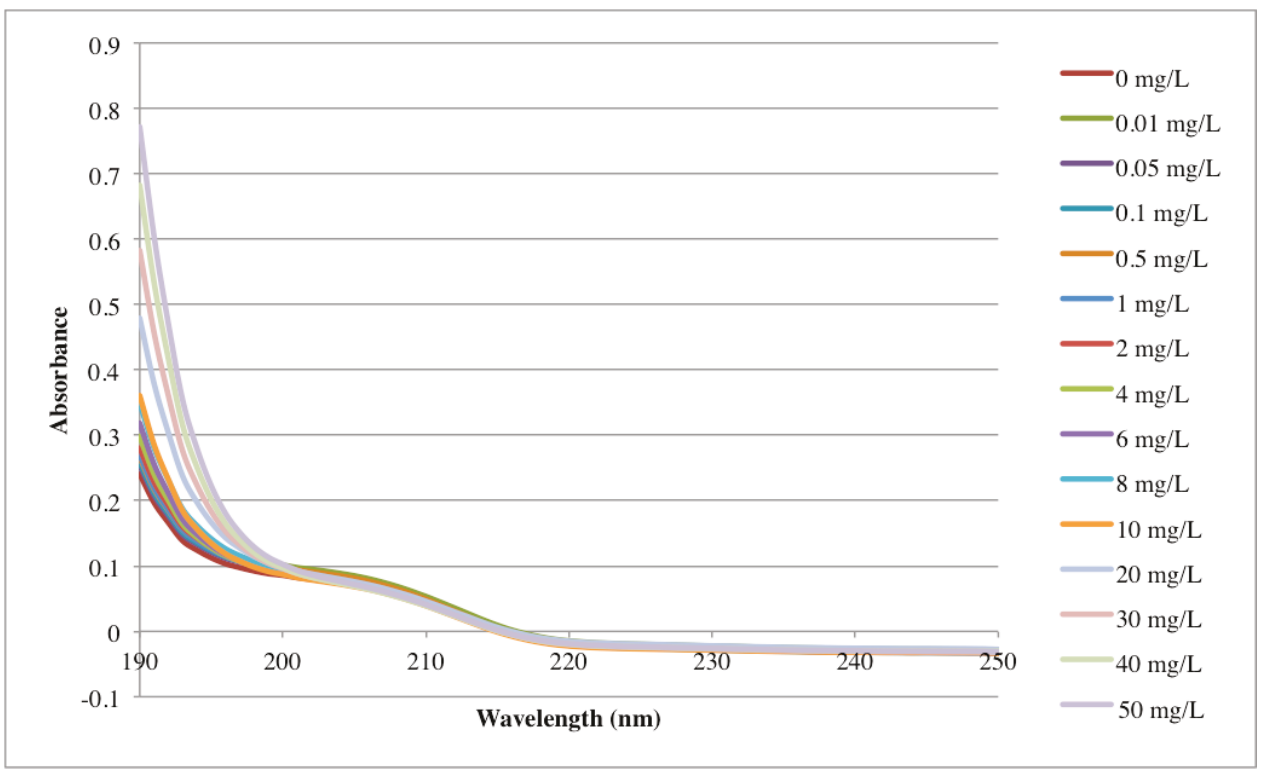

Figure 3.19 Absorbance spectra of $\mathrm{CaCl}_{2}$ in $5 \mathrm{mg} / \mathrm{L}$ solution of $\mathrm{SNF} 475$ (average of three measurements)

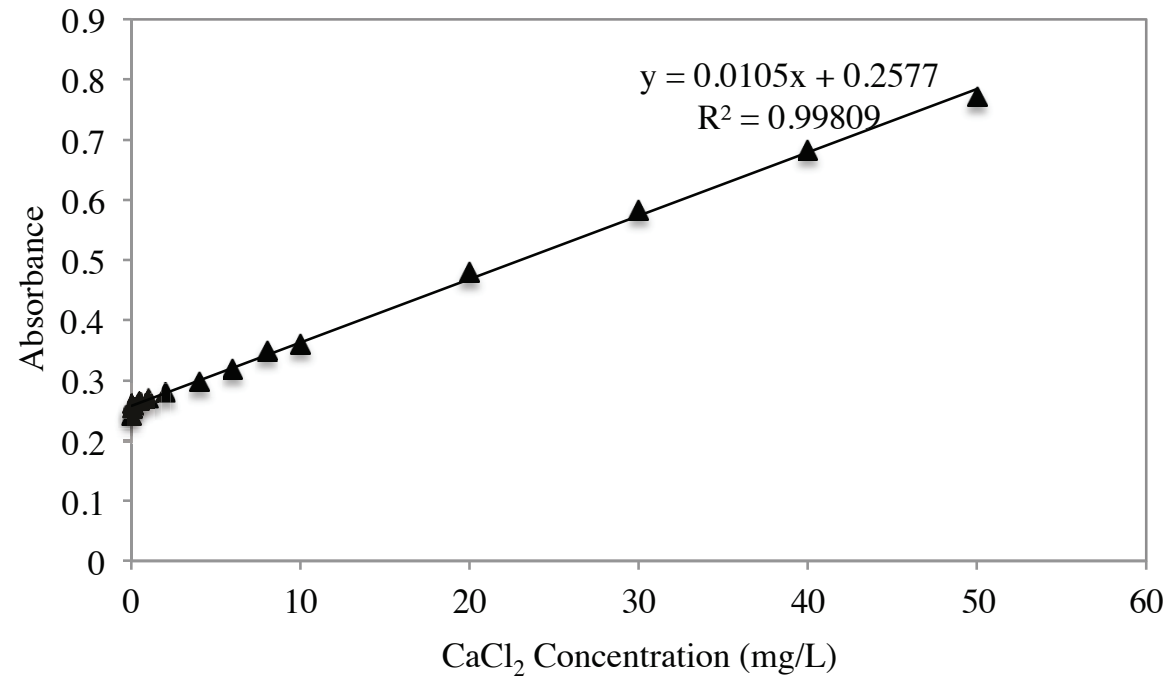

Figure 3.20 Relationship between $\mathrm{CaCl}_{2}$ concentration and absorbance in $5 \mathrm{mg} / \mathrm{L}$ solution of SNF475 at $\lambda=190 \mathrm{~nm}$ (average of three measurements) 


\subsubsection{Absorbance of Inorganic Conditioners}

\subsubsection{Alum}

The absorbance values for solutions of alum in deionized water over the range of $190-250 \mathrm{~nm}$ are provided in Figure 3.21, and it is seen that alum also appears in the ultraviolet spectra at wavelengths lower than $200 \mathrm{~nm}$. However, the concentration of alum needs to be higher than $50 \mathrm{mg} / \mathrm{L}$ in order to be detected at $190 \mathrm{~nm}$ (Figure 3.22). This is more easily observed in Figure 3.23 where the absorbance for $0-100 \mathrm{mg} / \mathrm{L}$ of alum samples is plotted against concentration. As shown in Figure 3.23, there is not much difference between the absorbance of deionized water alone, and deionized water samples containing alum up to $40 \mathrm{mg} / \mathrm{L}$.

It can therefore be concluded that the absorbance method at $190 \mathrm{~nm}$ can be used for detection of polymer within samples that are suspected to contain alum with concentrations lower than $50 \mathrm{mg} / \mathrm{L}$, without the need for establishing a new calibration curve. In samples containing higher alum doses, however, a new calibration curve should be established for accurate detection of polymers. In addition, the results also show that absorbance at $190 \mathrm{~nm}$ can be used for detection and quantification of relatively high concentrations of alum in water. 


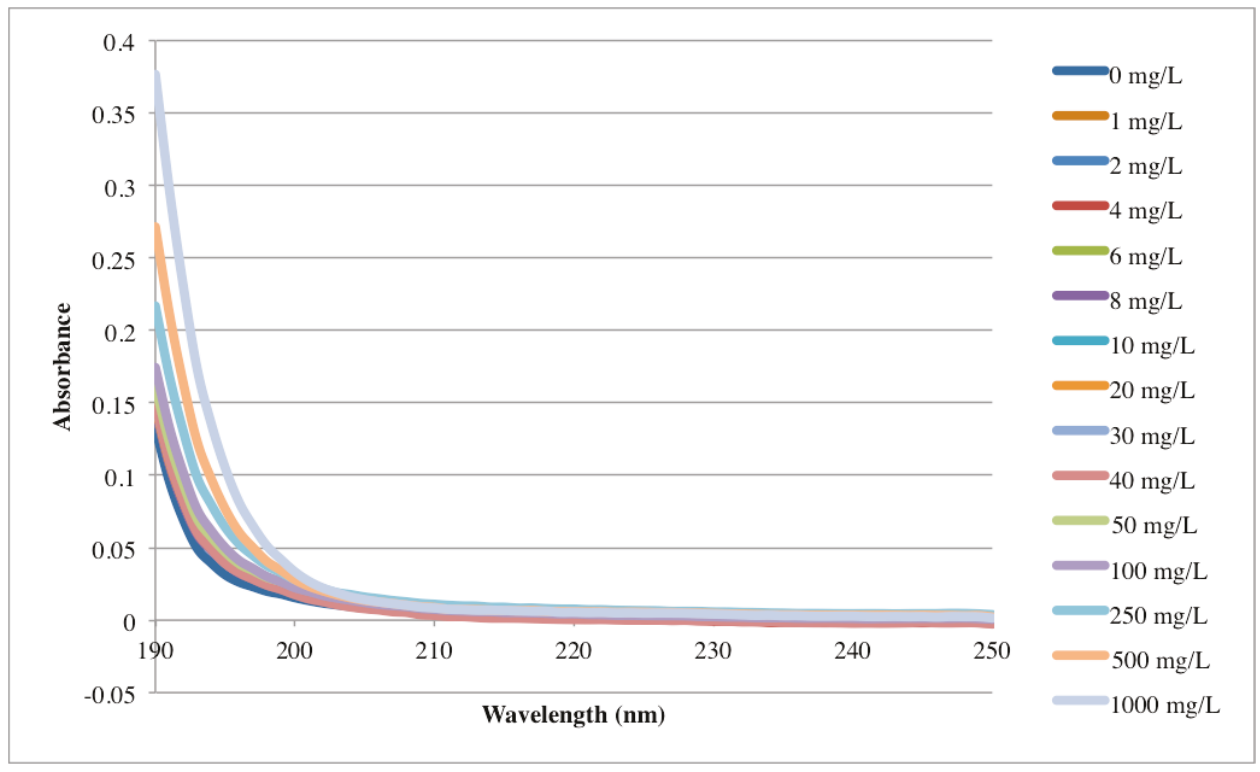

Figure 3.21 Absorbance spectra of alum in DW (average of three measurements)

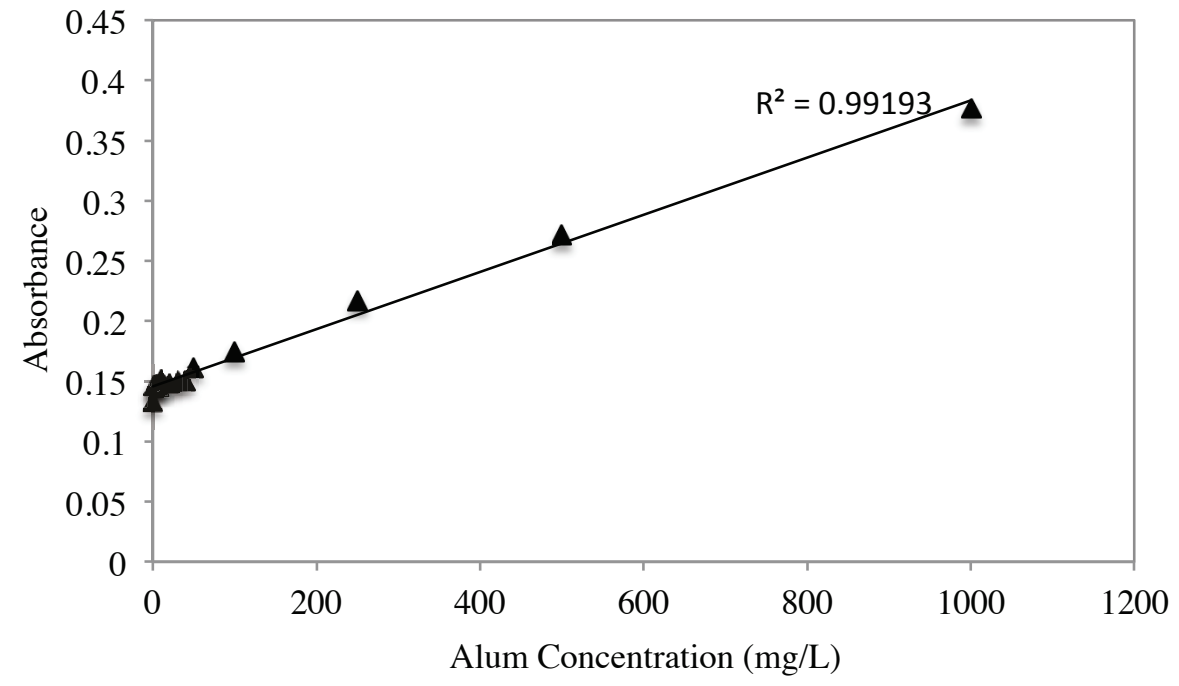

Figure 3.22 Relationship between alum concentration and absorbance in DW at $\lambda=190 \mathrm{~nm}$ (average of three measurements) 


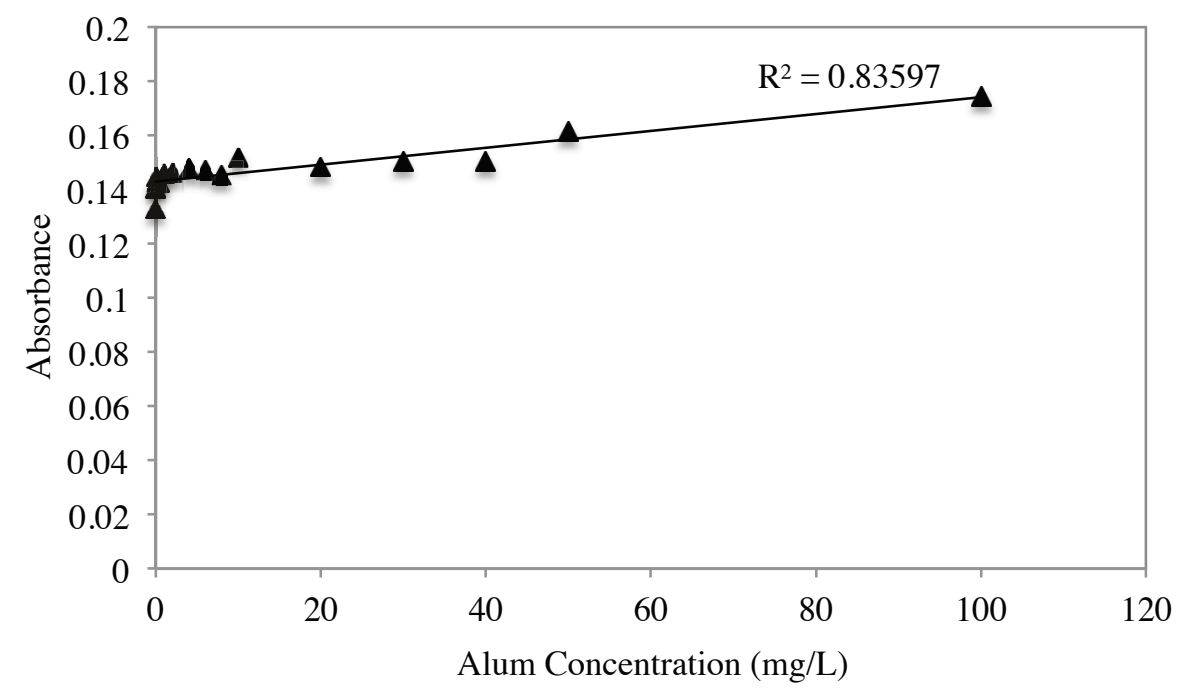

Figure 3.23 Relationship between alum concentration $(0-100 \mathrm{mg} / \mathrm{L})$ and absorbance in $\mathrm{DW}$ at $\lambda=190 \mathrm{~nm}$ (average of three measurements)

\subsubsection{Ferric Chloride and Ferrous Chloride}

The absorbance values for solutions of ferric chloride in deionized water over the range of $190-250 \mathrm{~nm}$ are provided in Figure 3.24. Similar to the salts and alum, ferric chloride appeared in the ultraviolet spectra at wavelengths lower than $200 \mathrm{~nm}$ with the peak of absorbance occurring at $190 \mathrm{~nm}$. Due to this reason, the absorbance values at this wavelength were plotted against ferric chloride concentration in Figure 3.25.

As opposed to ferric chloride, however, ferrous chloride absorbed ultraviolet light in the whole range of $190-250 \mathrm{~nm}$ as shown in Figure 3.26. Nonetheless, its peak of absorbance occurred at $190 \mathrm{~nm}$ same as ferric chloride. At $190 \mathrm{~nm}$, both ferric (Figure 3.25) and ferrous chloride (Figure 3.27) showed a linear relationship between absorbance and concentration, and were detected in concentrations as low as $1 \mathrm{mg} / \mathrm{L}$ and $0.5 \mathrm{mg} / \mathrm{L}$, respectively. Therefore, presence of ferric chloride and ferrous chloride would require establishing a new baseline before polymer measurement since they also strongly absorb 
light at $190 \mathrm{~nm}$. Furthermore, these results also show that UV-Vis spectrophotometry can be used for direct detection and quantification of residual ferric and ferrous chloride in water samples and at treatment plants.

It should be noted that in this study the interference of inorganic conditioners was monitored in deionized water as opposed to salts and $\mathrm{pH}$, which were inspected in $5 \mathrm{mg} / \mathrm{L}$ solution of SNF475 polymer in deionized water. The reason for this was to inspect whether UV-Vis spectroscopy can also be a potential tool for detection and quantification of inorganic conditioners in addition to their potential absorbance in the far UV region and thus interference on polymer spectra. However this inhibited monitoring any possible chemical reaction between the inorganic conditioners and synthetic polymers, which can be interesting and possibly subject of a future work. 


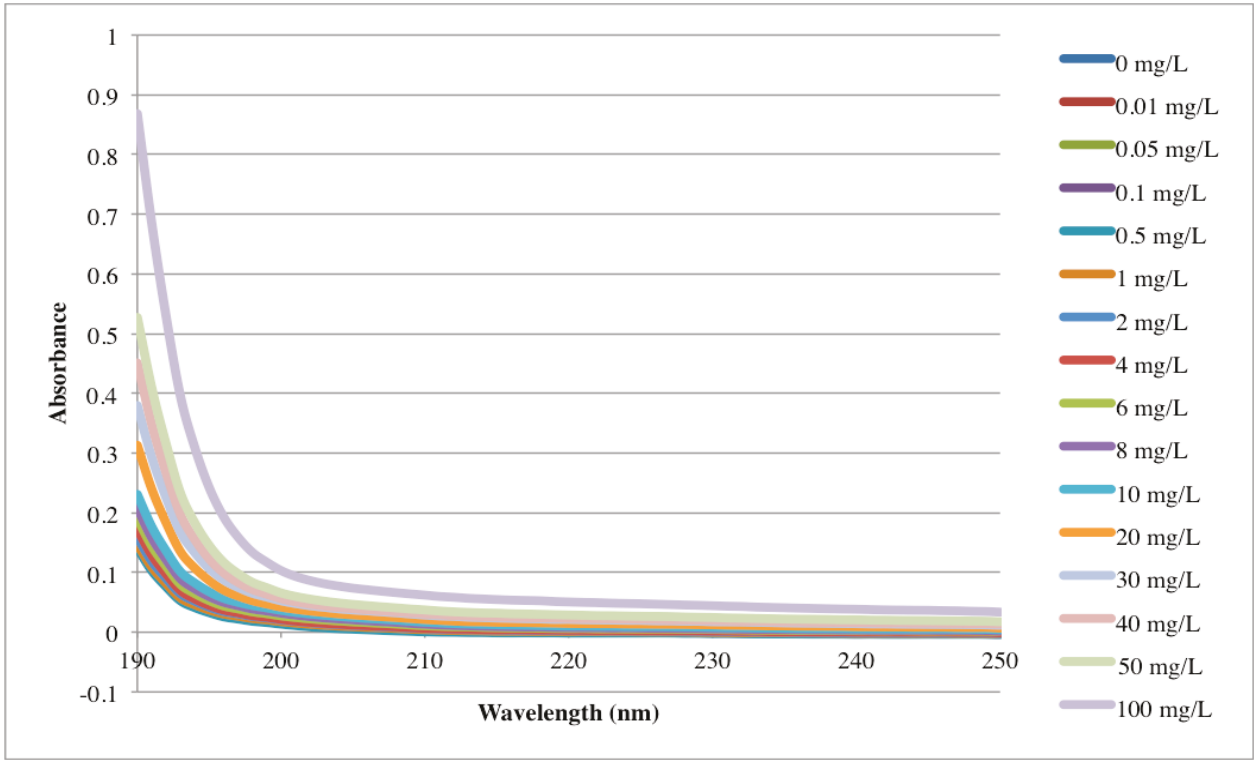

Figure 3.24 Absorbance spectra of ferric chloride in DW (average of three measurements)

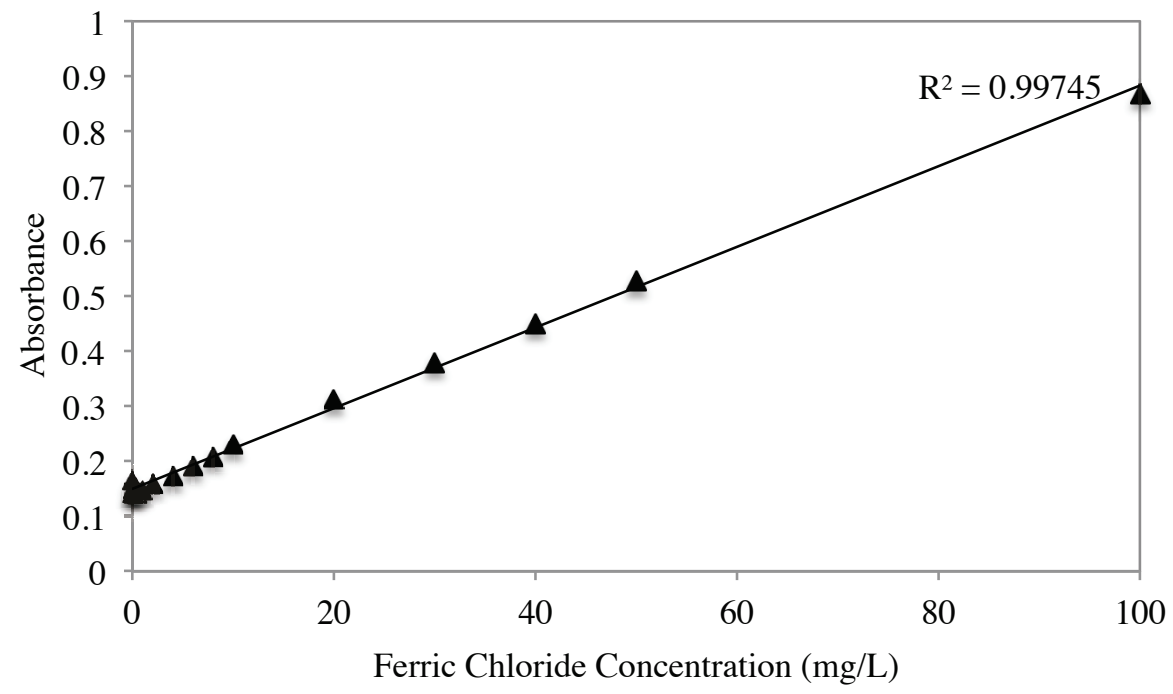

Figure 3.25 Relationship between ferric chloride concentration and absorbance in DW at $\lambda=190 \mathrm{~nm}$ (average of three measurements) 


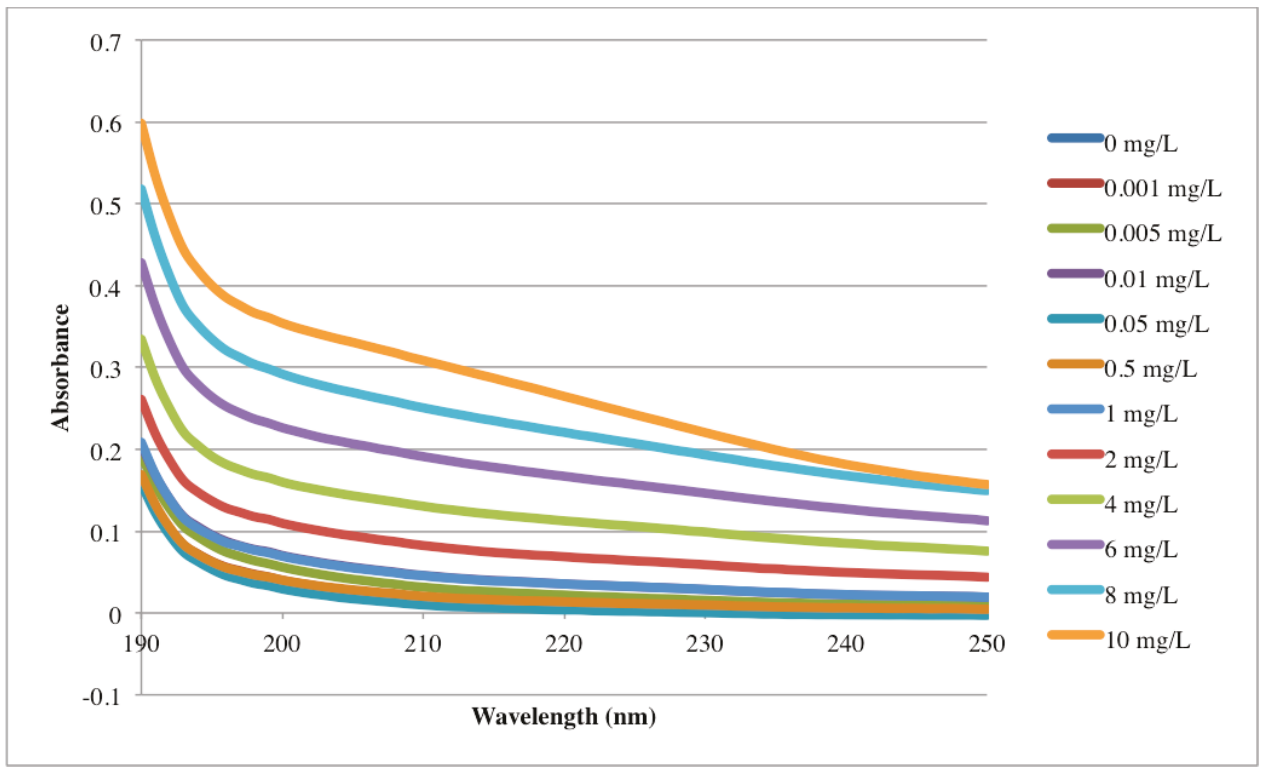

Figure 3.26 Absorbance spectra of ferrous chloride in DW (average of three measurements)

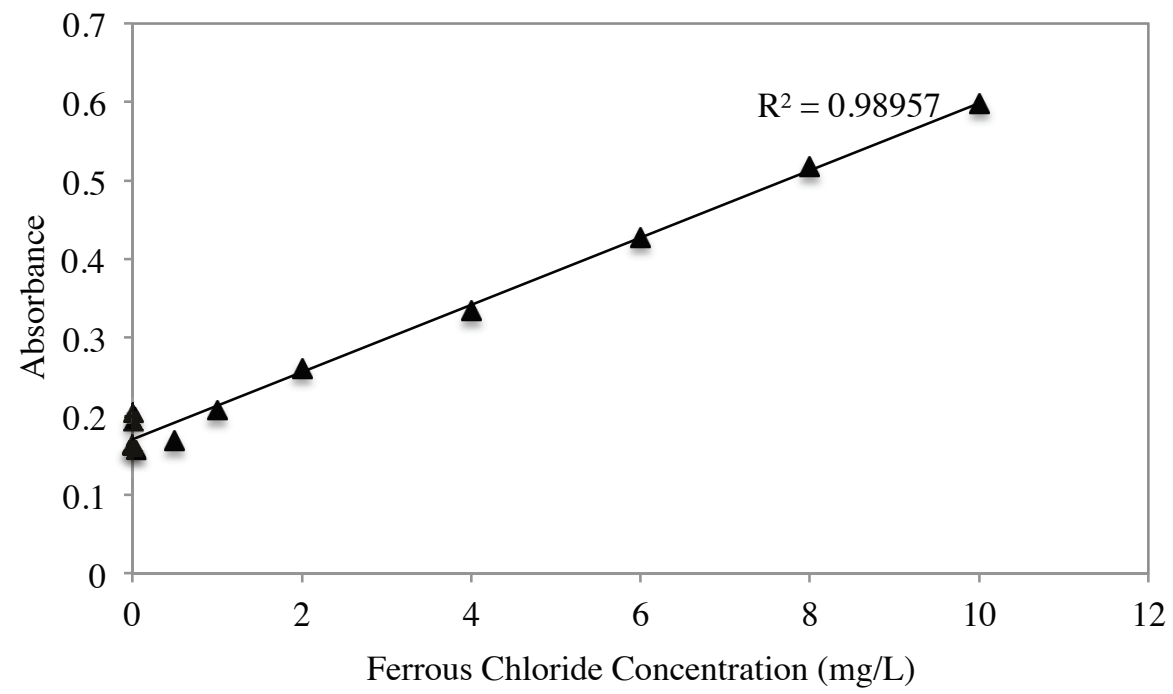

Figure 3.27 Relationship between ferrous chloride concentration and absorbance in DW at $\lambda=190$ nm (average of three measurements) 


\subsubsection{Suggestions to Minimize Interferences}

The similarity between the absorbance spectra of most of the chemicals studied in this chapter is that they strongly absorb light at $190 \mathrm{~nm}$ but their absorbance sharply drops at $200 \mathrm{~nm}$. As shown previously in Chapter 2, polymers absorb ultraviolet light at wavelengths lower than $230 \mathrm{~nm}$. Even though their peak absorbance is at $190 \mathrm{~nm}$, they still very strongly absorb light at $200 \mathrm{~nm}$. Therefore, in order to avoid the interference from salts, alum, and ferric chloride on the absorbance spectra of polymers, one can use the wavelength of $200 \mathrm{~nm}$ instead of $190 \mathrm{~nm}$ to construct calibration curves. The exception to this is ferrous chloride since it substantially absorbs light between 190 and $250 \mathrm{~nm}$. Wavelengths higher than $250 \mathrm{~nm}$ (Figure 3.26) would be required to avoid interference from ferrous chloride, and polyacrylamide polymers are not detectable at those wavelengths (Figures 2.2, and 2.5).

In order to show the effect of using $200 \mathrm{~nm}$ instead of $190 \mathrm{~nm}$ on the detection and quantification of polyacrylamide polymers, Figure 2.2 in Chapter 2 was revisited, and absorbance of Zetag8160 polymer was plotted against concentration at $200 \mathrm{~nm}$ (Figure 3.28). At $200 \mathrm{~nm}$, there was also a perfect linear relationship between concentration and absorbance. When the method detection limit was recalculated at 200 $\mathrm{nm}$ using Hubaux and Vos's method, a value of $0.4 \mathrm{mg} / \mathrm{L}$ was found as opposed to 0.2 $\mathrm{mg} / \mathrm{L}$ at $190 \mathrm{~nm}$. As expected, using $200 \mathrm{~nm}$ instead of $190 \mathrm{~nm}$ resulted in a slight decrease in the sensitivity and detection limit of the method.

The absorbance of SNF475 polymer at the wavelength of $200 \mathrm{~nm}$ versus its concentration also followed a linear trend (Figure 3.29). However, reduction in the sensitivity of SNF475 was higher and the method detection limit was increased to 2.6 
$\mathrm{mg} / \mathrm{L}$ at $200 \mathrm{~nm}$, which was $0.7 \mathrm{mg} / \mathrm{L}$ at $190 \mathrm{~nm}$. Compared to the 2-fold decrease in the sensitivity of Zetag8160 polymer, SNF475 polymer showed close to a 4-fold decrease in its sensitivity at $\lambda=200 \mathrm{~nm}$.

Therefore, detection of polymers at wavelengths under $230 \mathrm{~nm}$, instead of the preferred $190 \mathrm{~nm}$, is possible but the type of the polymer, detection limits, and peak absorbance of other chemicals present in water should be considered before choosing the ideal wavelength for the quantification of polyacrylamide polymers. Surface water and particularly wastewater may include many dissolved constituents from biological or chemical origin. Presence of these constituents would simply increase the baseline absorbance and accurate detection and quantification of polyacrylamide polymers can be achieved after establishing a calibration curve with this water or wastewater sample.

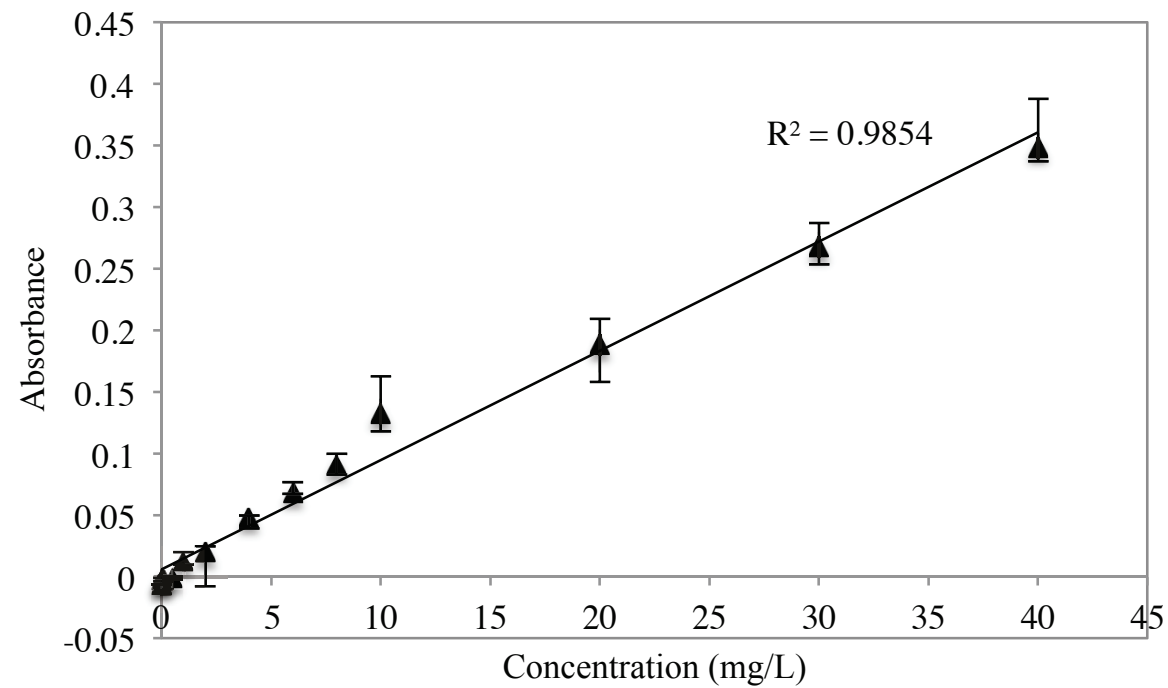

Figure 3.28 Relationship between Zetag8160 concentration and absorbance in DW at $\lambda=200 \mathbf{n m}$ (average of 3 standards) 


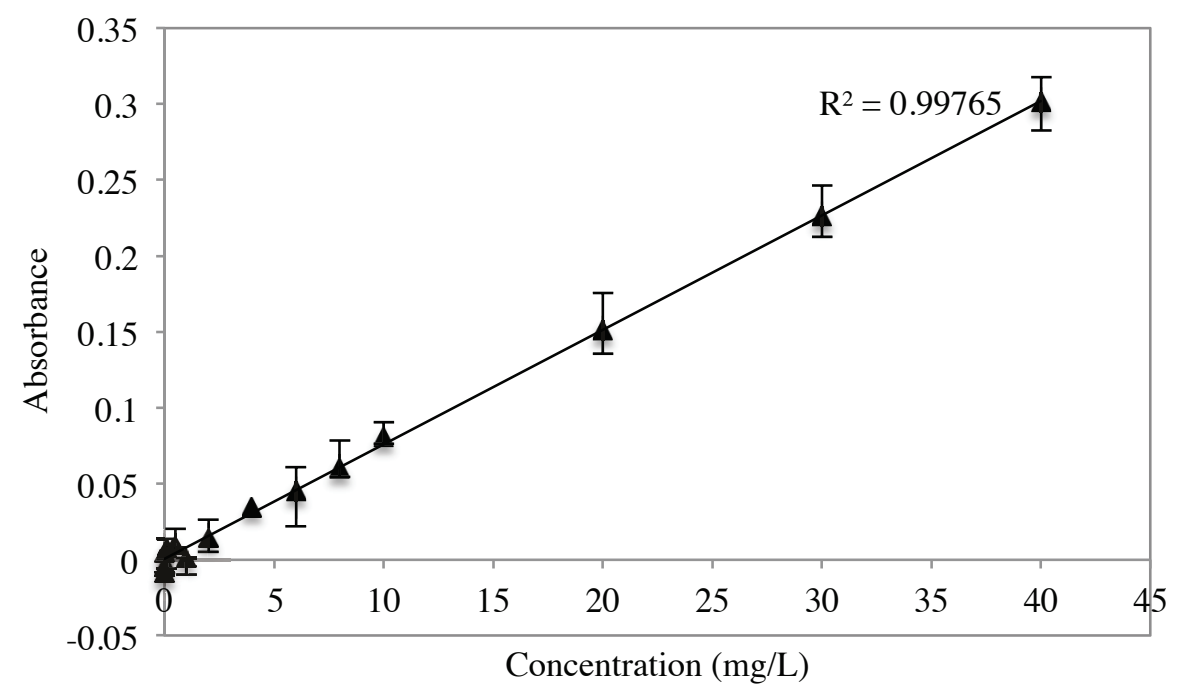

Figure 3.29 Relationship between SNF475 concentration and absorbance in DW at $\lambda=200 \mathrm{~nm}$ (average of 3 standards)

\subsection{Conclusions}

In this chapter, the effects of $\mathrm{pH}$ and two salts of $\mathrm{NaCl}$, and $\mathrm{CaCl}_{2}$ were investigated on the absorbance spectra of samples containing $5 \mathrm{mg} / \mathrm{L}$ of polyacrylamide polymer. In addition, the absorbance spectra of three commonly used treatment chemicals of alum, ferric chloride, and ferrous chloride in deionized water were studied.

The results showed that intense acidic $(\mathrm{pH}=3)$ and alkaline $(\mathrm{pH}=11)$ environments promote the hydrolysis of polyacrylamide and result in formation of a smaller peak at $\lambda=205 \mathrm{~nm}$ likely due to carboxylic acids. Furthermore, due to the appearance of $\mathrm{Cl}^{-}$and $\mathrm{OH}^{-}$ions in the ultraviolet spectra of water and similarity of the absorbance of water to that of polymers, it was concluded that the UV-Vis absorbance method would not work well in highly acidic and basic environments for solutions of $5 \mathrm{mg} / \mathrm{L}$ of polymer or lower because of the small differences between the absorbance of acidic/alkaline deionized water to that of deionized water samples containing polymer. 
As for the effects of salts on the absorbance of polyacrylamide, it was observed that any increase in the concentration of salts resulted in an increase in absorbance at wavelengths lower than $200 \mathrm{~nm}$ mainly due to the interference of chloride ions with the spectra, which was expected to occur, based on the study of Korshin et al. (1997).

As for alum, ferric chloride, and ferrous chloride, a linear relationship between the concentration and absorbance of those chemicals at wavelength of $190 \mathrm{~nm}$ was observed. For ferric chloride, and ferrous chloride, concentrations as low as 1 , and 0.5 $\mathrm{mg} / \mathrm{L}$ respectively could be detected in deionized water, whereas for alum, a change in the absorbance of deionized water was observed only when the concentration of alum was increased to $50 \mathrm{mg} / \mathrm{L}$. Therefore, ferric and ferrous chloride can change the baseline absorbance at $190 \mathrm{~nm}$ at low concentrations, but relatively high concentrations of alum (> $50 \mathrm{mg} / \mathrm{L}$ ) would be required to have a significant impact on absorbance at $190 \mathrm{~nm}$. More over, ferric chloride and alum absorbed light at wavelengths lower than $200 \mathrm{~nm}$, but ferrous chloride showed strong absorption up until $250 \mathrm{~nm}$.

Presence of $\mathrm{NaCl}, \mathrm{CaCl}_{2}$, alum, ferric chloride and ferrous chloride would increase the background absorbance at $190 \mathrm{~nm}$ but this would not affect the detection and quantification of polymers since their impact would be reflected in the calibration curves. Alternatively, interferences from salts, alum, and ferric chloride could be avoided by using absorption values at $200 \mathrm{~nm}$ instead of $190 \mathrm{~nm}$. 


\section{Chapter 4: Comparison of Polymer}

\section{Detection Methods}

\subsection{Introduction}

There are several methods reported in the literature for the detection and quantification of polymers. Overall, polymer detection methods can be divided in the four following groups with almost all of them having something in common which is the derivation of a compound from acrylamide and subsequent determination of that compound through the use of HPLC (high-performance liquid chromatography), GC (gas chromatography), ECD (electron-capture detection), and MS (mass spectroscopy) (Lu, Wu and Gan 2003). These four groups are as follows:

1. Methods based on chemical properties of amide or carboxyl groups in polyacrylamide including fluorescence spectrophotometry, amide hydrolysis with ammonia detection, and N-bromination method (Lu and Wu 2003).

2. Methods based on physical properties of polyacrylamide including viscosity measurement, flocculation based method, and size exclusion chromatography (Lu and $\mathrm{Wu}$ 2003).

3. Methods based on both chemical properties of amide groups and physical properties of polyacrylamide including colloid titration, turbidimetric method, and polarography ( $\mathrm{Lu}$ and $\mathrm{Wu} 2003)$.

4. Other methods including total organic carbon measurement, total nitrogen analysis, and radioactive labeling ( $\mathrm{Lu}$ and $\mathrm{Wu} 2003$ ). 
Methods based on chemical properties (\#1) are beneficial in the sense that they can have very low detection limits due to the use of chemical reactions. However, since these methods are based on the amide or carboxyl functional groups in polyacrylamide, they will only function well for samples that contain low amounts of those constituents due to the interference effects. However, dilution and/or centrifugation-filtration of the samples that contain high amounts of amide or hydroxyl groups could help reduce those effects by removal of solids and to enable chemical methods to detect polymer concentration in such samples.

Physical methods (\#2) have much simpler procedures compared to chemical methods and are less labor extensive. However, they have much higher detection limits compared to that of chemical methods. Consequently, these methods could be used to measure polymer concentrations in samples with higher polymer doses.

Methods in categories \#3, and \#4 have not found much practicality in industry as some of these methods are very complicated and labor extensive while the others quantify for the concentration of polyacrylamide indirectly i.e., they are based on measuring the total number of carbon or nitrogen atoms. Due to this reason and also due to the limited nature of physical methods to concentrated samples, two methods of amide hydrolysis and N-Bromination were chosen from the category of chemical methods (\#1) as a comparison with the UV-Vis absorbance method developed in this study. Another distinction between these two methods and other methods reported in literature is that they also use UV-Vis spectroscopy to detect and quantify polyacrylamide as opposed to other methods that use NMR, size exclusion, or fluorescence spectroscopy. These two methods are presented in detail in the following sections. 


\subsubsection{Acidic/Alkaline Hydrolysis (or Dye Complexation)}

Hydrolysis in acidic or alkaline solutions is known to be the least time consuming and labor extensive and has rather high accuracy compared to other chemically based methods in group \#1 (Chmilenko, Korobova and Nazarenko 2004). It is also known to have very low detection limits of $0.02 \mathrm{ppm}(\mathrm{Lu}$ and $\mathrm{Wu} 2003)$ and is comprised of the following three steps:

1. Hydrolysis of polyacrylamide in either acidic or alkaline solutions to increase its reactivity.

2. Addition of the hydrolyzed polymer to cationic salts.

3. Measurement of the change in the salt's absorbance caused by polymer addition, via the use of a spectrophotometer.

\subsubsection{Hydrolysis of Polymers}

Polyacrylamides come in three categories of cationic, anionic, and neutral. This step is necessary only if the polymer that is being used is neutral. When the polymer is free of charge, it will not react upon contact with other substances such as cationic salts, which is fundamental for this method to work. However, once it is put in acidic or alkaline solutions, some of the monomers in the polymeric chain, take up the form of an ion once they react with the present acid or base and become negatively or positively charged. Depending on the monomers that build up the polymer, hydrolysis in either acidic or basic environment might be preferred. But for the case of polyacrylamides, because of the complicated effect of imidization, the acidic hydrolysis method has not found practical application for obtaining hydrolyzed derivatives of polyacrylamide and alkaline hydrolysis is mostly used (Chmilenko, Korobova and Nazarenko 2004). 
The sensitivity of this method decreases with the increasing degree of polyacrylamide hydrolysis, which lowers the ratio of amide groups to carboxylic groups in the polyacrylamide molecule and inhibits the polymer to function properly $(\mathrm{Lu}$ and $\mathrm{Wu}$ 2003). Therefore time of contact as well as the concentration of the alkaline solution is important.

However, in the case of wastewater treatment plant polymers, this step is not required since the polymers are already positively charged to coagulate with the negatively charged solids in the municipal wastewater and therefore are not neutral.

\subsubsection{Absorbance Measurement of Salts Before and After Polymer Addition via Spectrophotometer}

The salts on their own show different absorbance curves at different $\mathrm{pH}$ values due to the differences in their dissociation, and as dissociation is higher, absorbance will also increase due to the existence of more ions. The reaction of hydrolyzed polymers with these ions will result in the formation of uncharged molecules, which do not contribute to the absorbance and thus result in a decrease in the absorbance curve of the salt. This decrease in absorbance is measured as the concentration of polymer is increased at a constant $\mathrm{pH}$ and the results turn into linear plots with $\mathrm{x}$ axis equal to the molar ratio of polymers to salts and y axis equal to absorbance of the peak. These peaks can readily be used later on for samples with known absorbance to find the concentration of polymer.

\subsubsection{N-Bromination Method and Spectrophotometry of Starch Complex}

$\mathrm{N}$-Bromination is one of the favored methods in the sense that it has a low

detection limit $(0.1 \mathrm{mg} / \mathrm{L})$ and is invulnerable to most of the common ions except chlorine ( $\mathrm{Lu}$ and $\mathrm{Wu} 2003$ ). It involves addition of bromine, sodium formate, and starch- 
$\mathrm{CdI}_{2}$ to a solution of known polyacrylamide concentration in a timely manner (i.e., adding each reagent and waiting a couple of minutes prior to addition of other reagents) and measuring the absorbance of the final starch complex product via spectrophotometer. The following series of reactions are suspected to occur for the final starch complex to be produced (Scoggins and Miller 1975):

1. Bromination of amide group in a (buffered) solution with known concentrations of polyacrylamide in order to increase its reactivity:<smiles>[R]C(=O)N[B-]Br</smiles>

- The buffer solution is added in order to keep $\mathrm{pH}$ about the same level because $\mathrm{pH}$ (hydrogen ion concentration) affects reactions occurring in steps 2,3, and 4 below.

2. Destruction of excess bromine via sodium formate in order to prevent high results prior to addition of iodide ion:

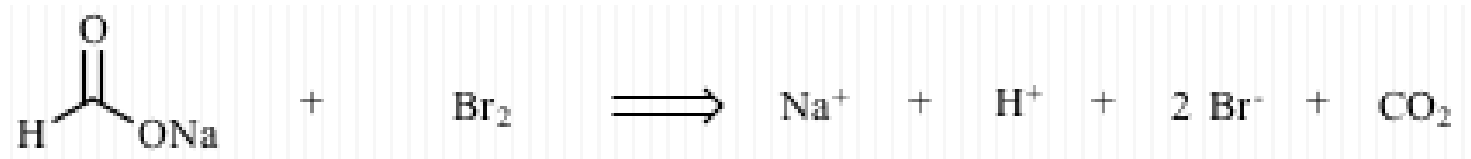

- This step might be tricky in the sense that both the concentration of sodium formate and the reaction time affects the absorbance of final product and therefore the tests must be very consistent with the procedure taken to produce concentration-absorbance curves.

3. Production of hypobromous acid via hydrolysis of the brominated amide: 


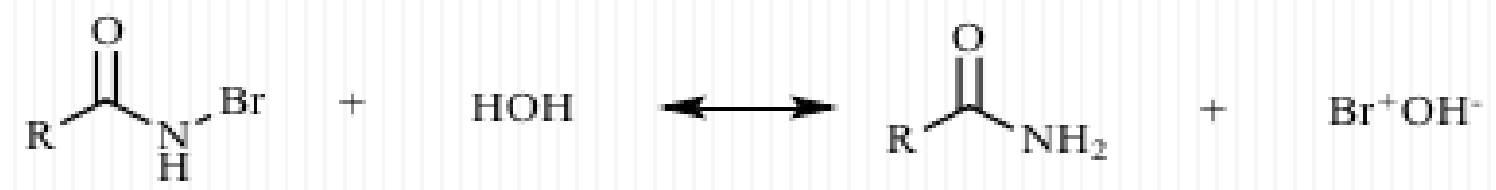

4. Production of iodine via the reaction of hypobromous acid with iodide ion produced from deionization of starch-CdI ${ }_{2}$ reagent:

$$
\mathrm{Br}^{+} \mathrm{OH}^{-}+\mathrm{I}^{-}+2 \mathrm{H}^{+} \rightarrow \frac{1}{2} \mathrm{I}_{2}+\mathrm{HBr}+\mathrm{H}_{2} \mathrm{O}
$$

5. And finally production of the starch complex via reaction of iodine, iodide ion, and deionized starch and measuring its absorbance at a wavelength of $610 \mathrm{~nm}$ :

$$
\mathrm{I}_{2}+\mathrm{I}^{-}+\text {Starch } \rightarrow \mathrm{I}_{3}^{-}-\text {Starch (Blue Complex) }
$$

The steps specified above must be carried out several times with different concentrations of polyacrylamide. The measured absorbance at $610 \mathrm{~nm}$ should then be plotted with the corresponding polymer concentration. These individual dots will result in a straight line or a curve which helps find the polymer concentration in a sample upon measurement of its absorbance.

As mentioned before, existence of chlorine ion interferes with absorption of starch complex but this effect can be inhibited via maintaining the $\mathrm{pH}$ at a value of 4 or lower for the samples that are suspected to include chlorine concentration of $1000 \mathrm{ppm}$ or higher (Scoggins and Miller 1979). Oil is also known to cause interference using this procedure and it should be removed prior to bromination through filtration or by extraction using chloroform (Scoggins and Miller 1979). In order to correct for the effects of dissolved organic matter (DOM), Lu and $\mathrm{Wu}$ (2003) adopted the N-Bromination method but they also did a second spectrophotometric determination at wavelength of 
$254 \mathrm{~nm}$ to correct the interference from DOM. But this is only valid if the DOM are homogeneous and there is not much variability in them ( $\mathrm{Lu}, \mathrm{Wu}$ and Gan 2003).

The goal of this chapter was to compare the performance of the UV-Vis based detection method developed in this thesis to two of the established methods (dye method and N-Bromination) reported in the literature. Detection limits for the three methods were established and compared in a range of water matrices (deionized water, river water, and wastewater) using Hubaux and Vos's method explained in Chapter 2 of this thesis.

\subsection{Materials and Methods}

\subsubsection{Water Samples}

In order to compare the sensitivity of absorbance method with the dye method and N-Bromination method, river water, and secondary wastewater (from ROPEC) were filtered and diluted as indicated in the next paragraph. Deionized water was used as is.

The Rideau River water sample, and secondary wastewater (with HRT of 4-6 hours, influent flow recycle ratio of about $58 \%$, and SRT of about 5 days) were first filtered through $0.45 \mu \mathrm{m}$ filter. Before usage, river water and secondary wastewater were diluted with deionized water at a ratio of $2 \mathrm{v}$ river water/wastewater: $1 \mathrm{v}$ deionized water.

\subsubsection{Polymer Stock Solution}

Polymer stock solutions of $500 \mathrm{mg} / \mathrm{L}$ or $0.05 \%$ were prepared as follows: in a plastic weighing dish, $0.5 \mathrm{~g}$ of Zetag8160 (polyacrylamide, medium-high cationic charge, high molecular weight, granular solid form) was weighted using a scale (SI-114, Denver Instruments, Canada), and decanted into a $1 \mathrm{~L}$ glass beaker. About $1 \mathrm{~L}$ of deionized water was also added to the polymer. The mixture was stirred using a jar tester (Phipps and 
Bird, USA) at $200 \mathrm{rpm}$ for 5 minutes followed by 55 minutes at $125 \mathrm{rpm}$ and was allowed to rest for 60 minutes prior to usage.

\subsubsection{Dye-complexation}

Shen et al. (2006) introduced about 10 anionic salts (that would coagulate with positively charged polymers to make neutral flocs that do not absorb ultraviolet light), out of which, Direct Light Resistance Black G (DG) with color index of Direct Black 19 (35255), was chosen based on inventory of Sigma Aldrich. Stock solutions of $50 \mathrm{mg} / \mathrm{L}$ Direct Light Resistance Black $G$ dye was then prepared in each of the samples of deionized water, river water and secondary wastewater.

In 6 beakers of $250 \mathrm{~mL}, 200 \mathrm{~mL}$ of dye solution in deionized water was decanted. Enough polymer from the polymer stock solution was then added to the beakers such that they had polymer concentrations of $0,0.1,0.5,1,5$, and $10 \mathrm{mg} / \mathrm{L}$. The beakers were then stirred at $200 \mathrm{rpm}$ for two minutes followed by $50 \mathrm{rpm}$ for 10 minutes and were left over night (Shen, Ren and Zhuang 2006). The next morning, the absorbance of the supernatant of each of the samples (taken 2-3 cm below the surface) was measurement over a range of $190-800 \mathrm{~nm}$ via UV-Vis Spectrophotometer (Cary 100 Bio UV-Vis Spectrophotometer, Varian Inc./Aligant Technologies, Canada) and the results were

recorded via Scan software (Cary WinUV, Varian Inc., Canada). The same procedure was performed with dye solution that was made in other water matrices of river water and wastewater. 


\subsubsection{N-Bromination}

The N-Bromination was an intensive method in terms of workload, and the procedure for it was closely followed from Lambert and Zitomer (1963) as well as Scoggins and Miller (1975).

In six $50 \mathrm{~mL}$ volumetric flasks, $5 \mathrm{~mL}$ of sodium acetate-acetic acid buffer with $\mathrm{pH}=5.5$ was added followed by $30 \mathrm{~mL}$ of deionized water. From polymer stock solution, enough polymer was then added to each volumetric flask such that they had final concentrations of $0,0.1,0.5,1,5$, and $10 \mathrm{mg} / \mathrm{L}$. Thereafter, $1 \mathrm{~mL}$ of a solution of saturated bromine water was added to each flask. The flasks were then stirred and allowed to react for 5 minutes. After the wait time, $5 \mathrm{~mL}$ of $1 \%$ (by mass) solution of sodium formate was added to each flask. The flasks were then stirred and were allowed to react for 2 more minutes. Finally, $5 \mathrm{~mL}$ of a solution of starch-CdI $\mathrm{C}_{2}$ was added to each flask, followed by dilution to a volume of $50 \mathrm{~mL}$. After stirring and an additional 10 minutes of reaction time, the absorbance of the solutions was measured via UV-Vis spectrophotometer over a range of $190-800 \mathrm{~nm}$. The same procedure was repeated for other water matrices of river water and wastewater. However, the final dilution was done by deionized water in all water matrices.

\subsubsection{Preparation of Starch-CdI 2 Solution}

In a $2 \mathrm{~L}$ volumetric flask, added $400 \mathrm{~mL}$ of deionized water followed by $11 \mathrm{~g}$ of cadmium iodide and allowed the solution to boil gently for 15 minutes. The solution was then diluted to a volume of $800 \mathrm{~mL}$ followed by addition of $15 \mathrm{~g}$ of superlose while stirring. After an additional 10 minutes of boiling, the solution was filtered through Whatman's No. 42 (fine) filter paper on a butcher funnel. This step was done in less than 
1 hour to ensure the solution is remained well above $65^{\circ} \mathrm{C}$. The filtrate was then diluted to a volume of $1 \mathrm{~L}$ and was allowed to cool before utilization.

\subsubsection{Preparation of Saturated Bromine Water}

Preparation of saturated bromine water was done by adding $1.128 \mathrm{~mL}$ of liquid bromine to $96.5 \mathrm{~mL}$ of deionized water in a fume hood. For best results the bromine was added very slowly and the solution was under constant agitation.

\subsubsection{Absorbance Method}

As opposed to N-Bromination method and the dye method, the absorbance method is much easier to carry out.

In six $50 \mathrm{~mL}$ centrifuge tubes, required volume of Zetag8160 stock solution was added such that the final solutions had polymer concentration equal to $0,0.1,0.5,1,5$, and $10 \mathrm{mg} / \mathrm{L}$ after dilution with deionized water up to a volume of $50 \mathrm{~mL}$. After stirring the content of the centrifuge tubes, their absorbance values were measured via UV-Vis spectrophotometer over a range of 190-250 $\mathrm{nm}$. The same steps were repeated with other water matrices of river water and wastewater.

\subsection{Results and Discussion}

\subsubsection{Detection of Polymer in Deionized Water}

Figure 4.1 demonstrates the absorbance spectra of different Zetag8160 doses in deionized water over wavelengths of 190-800 nm using the dye method. The peak absorbance values at $\lambda=646 \mathrm{~nm}$ versus polymer doses for the dye method are provided in Figure 4.2. The corresponding figures for N-Bromination method (at $200 \mathrm{~nm}<\lambda<800 \mathrm{~nm}$ with peak of absorbance at $580 \mathrm{~nm}$ ), and absorbance method (at $190 \mathrm{~nm}<\lambda<250 \mathrm{~nm}$ with peak of absorbance at $190 \mathrm{~nm}$ ) are provided in Figures 4.3 through 4.6. 
As shown in Figures 4.2, 4.4, and 4.6, the changes in peak absorbance values occurs shortly after the introduction of polymer, i.e. at a dose of about $0.1 \mathrm{mg} / \mathrm{L}$ for dye method, $0.3 \mathrm{mg} / \mathrm{L}$ for $\mathrm{N}$-bromination method, and $0.1 \mathrm{mg} / \mathrm{L}$ for absorbance method.

Using the Hubaux and Vos's procedure provided in Chapter 2, method detection limit associated with dye, N-Bromination, and absorbance methods were calculated to be $0.6,0.5$, and $0.2 \mathrm{mg} / \mathrm{L}$ respectively, indicating that the absorbance method is more sensitive compared to dye and N-Bromination methods.

The expected MDL for N-Bromination and dye methods were 0.1 , and $0.02 \mathrm{mg} / \mathrm{L}$ respectively as discussed in previous sections. The difference between the calculated MDL $(0.5 \mathrm{mg} / \mathrm{L})$ and the one from literature $(0.1 \mathrm{mg} / \mathrm{L})$ for $\mathrm{N}$-Bromination method is not significant and can be due to the differences between the chemicals used. However, the difference between literature MDL $(0.02 \mathrm{mg} / \mathrm{L})$ and calculated MDL $(0.6 \mathrm{mg} / \mathrm{L})$ for dye method is much higher, and calculated MDL is about 1 order of magnitude higher than that of literature MDL. One reason for this high difference can be the fact that the expected MDL for dye method was extracted from a paper that uses cationic dyes to react with anionic polymer, whereas in this study anionic dye was used to react with cationic polymer. Another reason can be the fact that there are a variety of salts to be used for this purpose and each one of them can result in different detection limits. In addition, use of different polymers can also affect the detection limits, as each polymer has different charge, and molecular weight.

It should be noted that not all of the data points provided in the calibration curves below were used for calculation of the method detection limits as some of the points deviated from regression line and resulted in a significant increase in the detection limit. 
Due to this reason, the data in the lower concentration range were used mostly for calculating method detection limit.

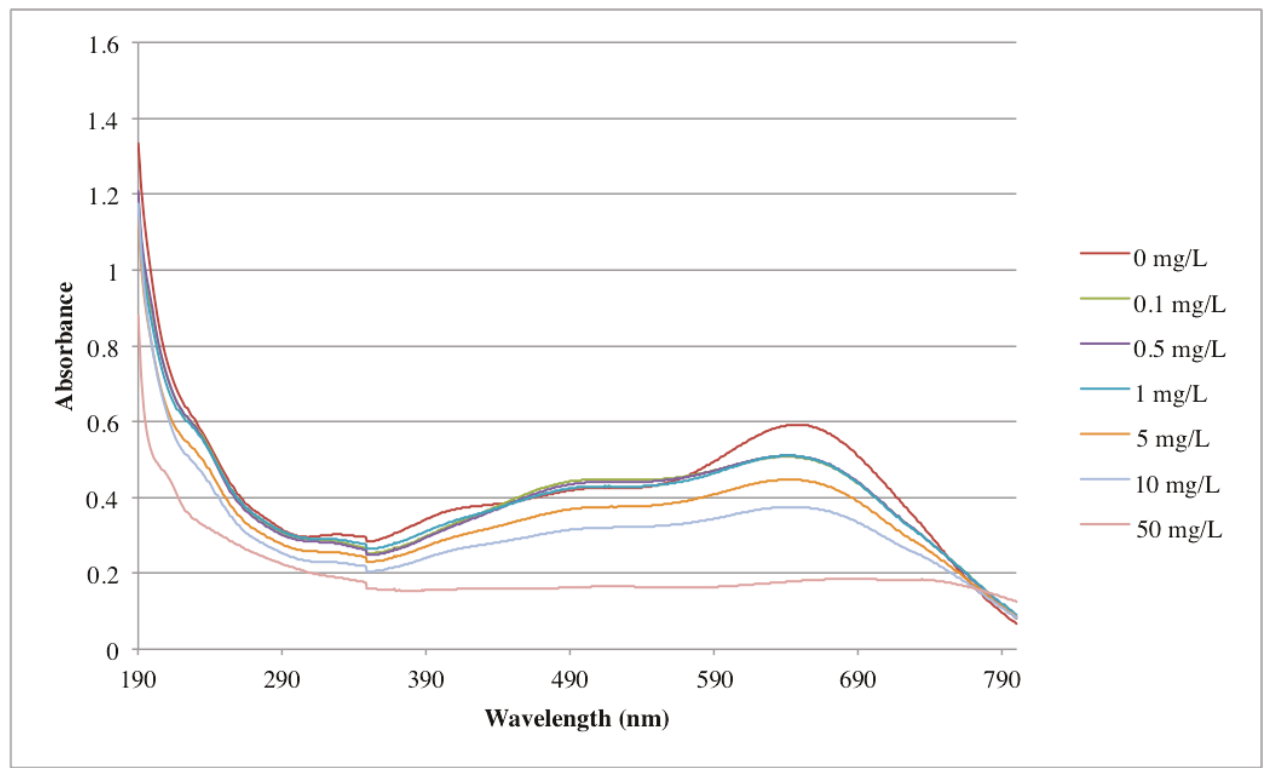

Figure 4.1 Absorbance values of different Zetag8160 concentrations in DW from $\lambda=190$ to $800 \mathrm{~nm}$ using the dye method (average of three measurements)

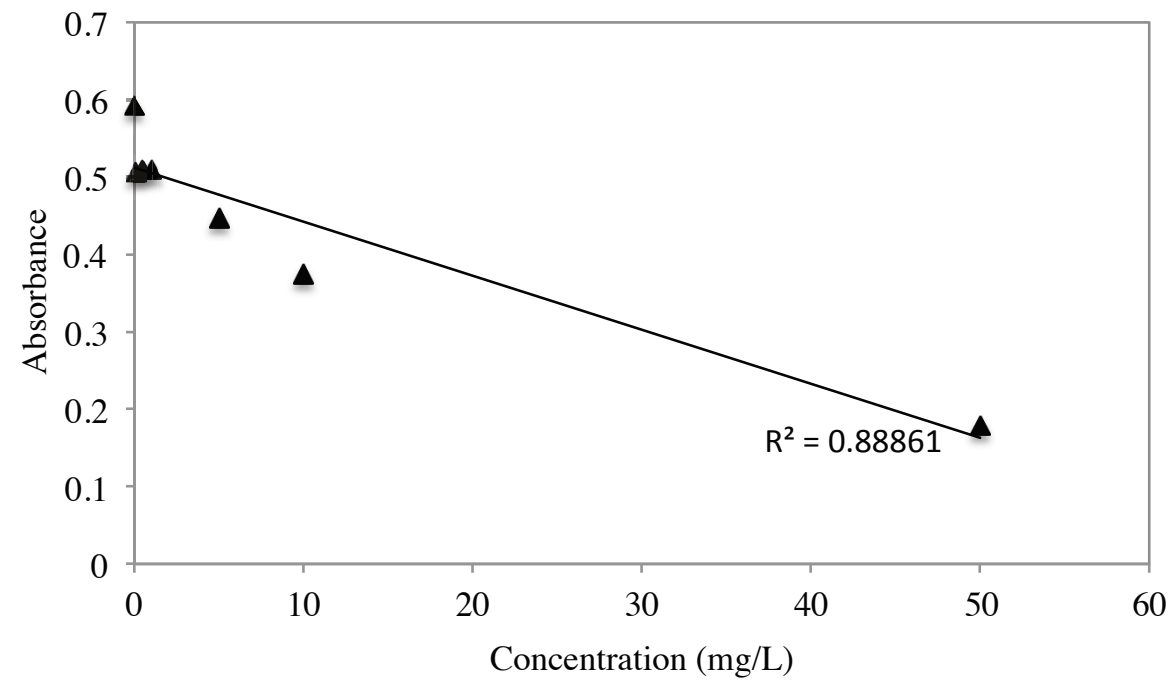

Figure 4.2 Absorbance values of Zetag8160 in DW at $\lambda=646 \mathrm{~nm}$ using the dye method (average of three measurements) 


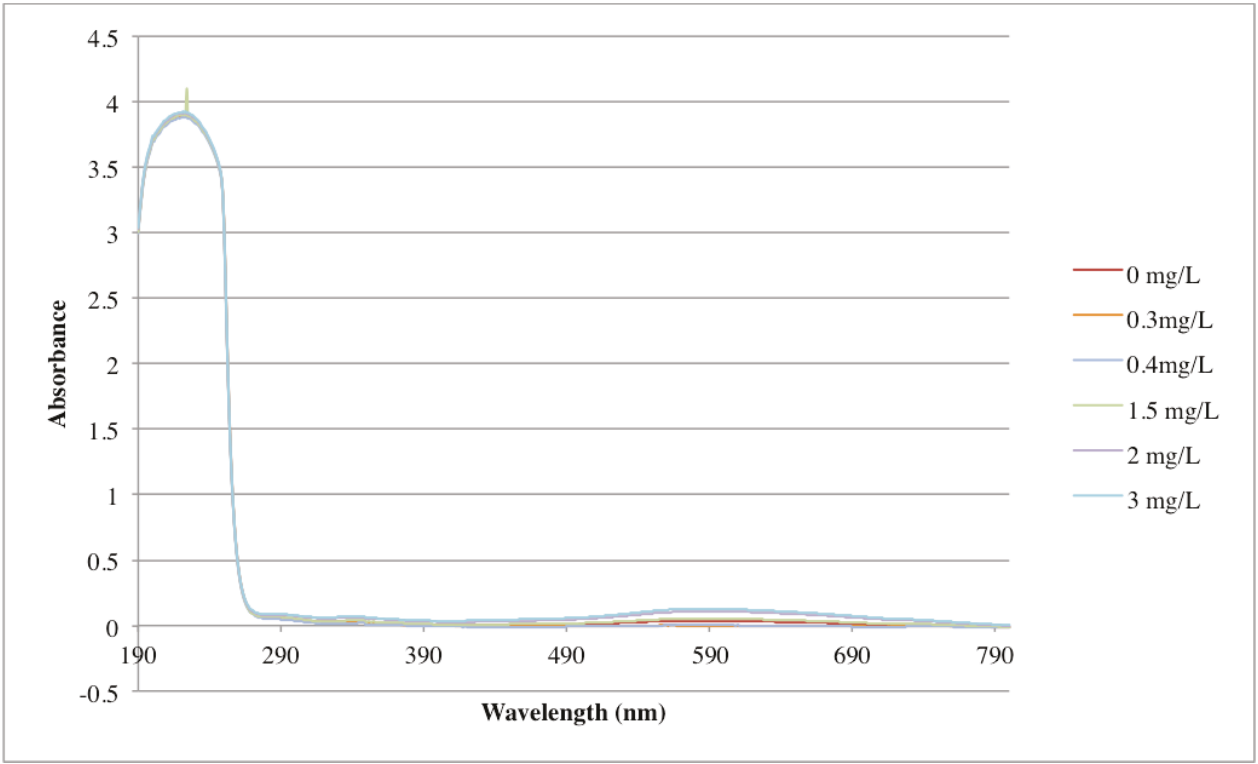

Figure 4.3 Absorbance values of different Zetag8160 concentrations in DW from $\lambda=190$ to $800 \mathrm{~nm}$ using the N-Bromination method (average of three measurements)

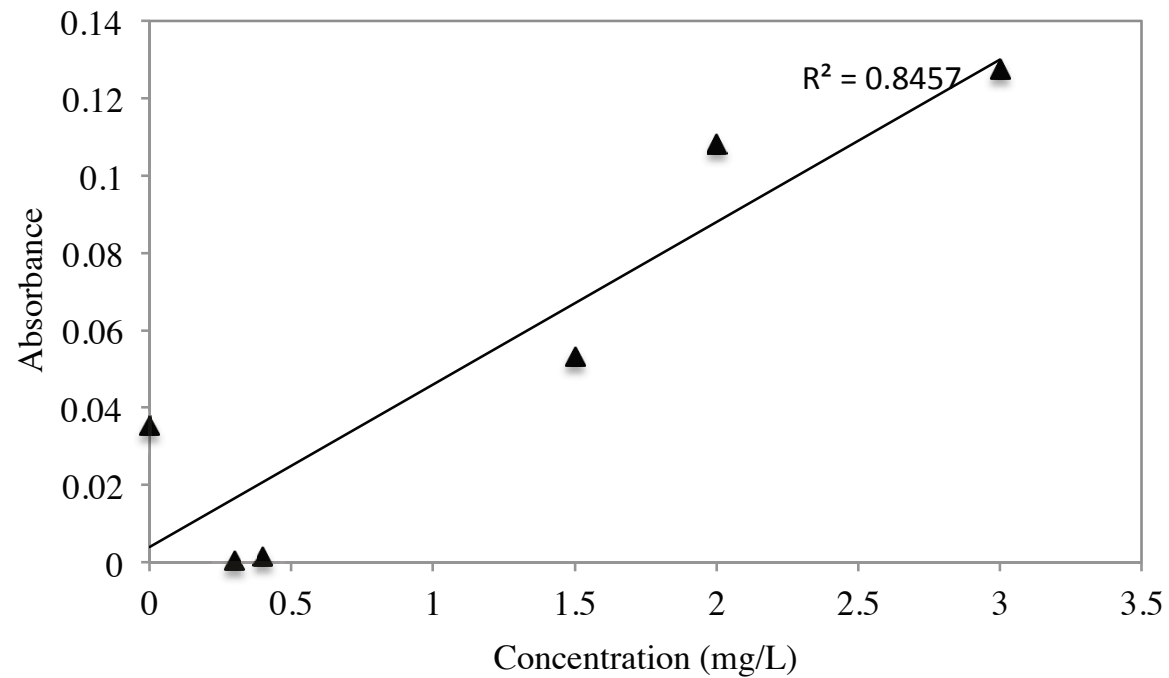

Figure 4.4 Absorbance values of Zetag8160 in DW at $\lambda=580 \mathrm{~nm}$ using the $\mathrm{N}$-Bromination method (average of three measurements) 


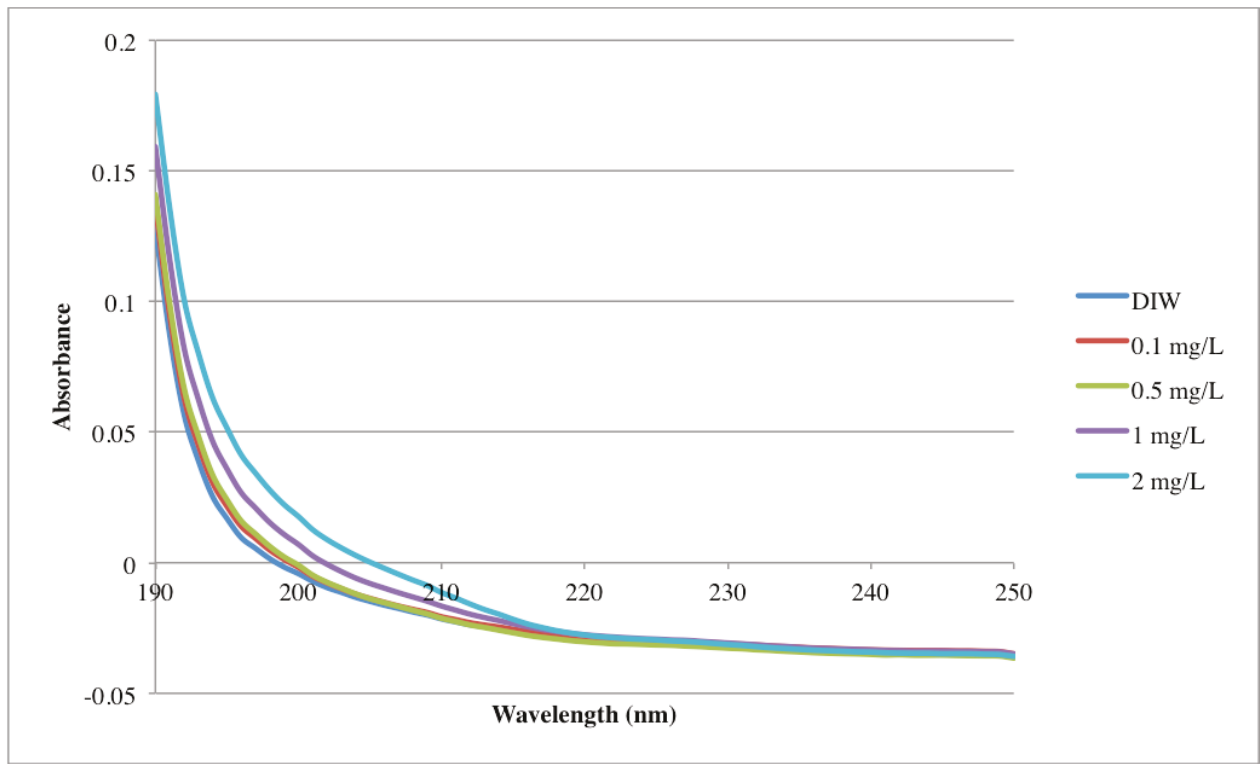

Figure 4.5 Absorbance values of different Zetag8160 concentrations in DW from $\lambda=190$ to $250 \mathrm{~nm}$ using the absorbance method (average of three standards)

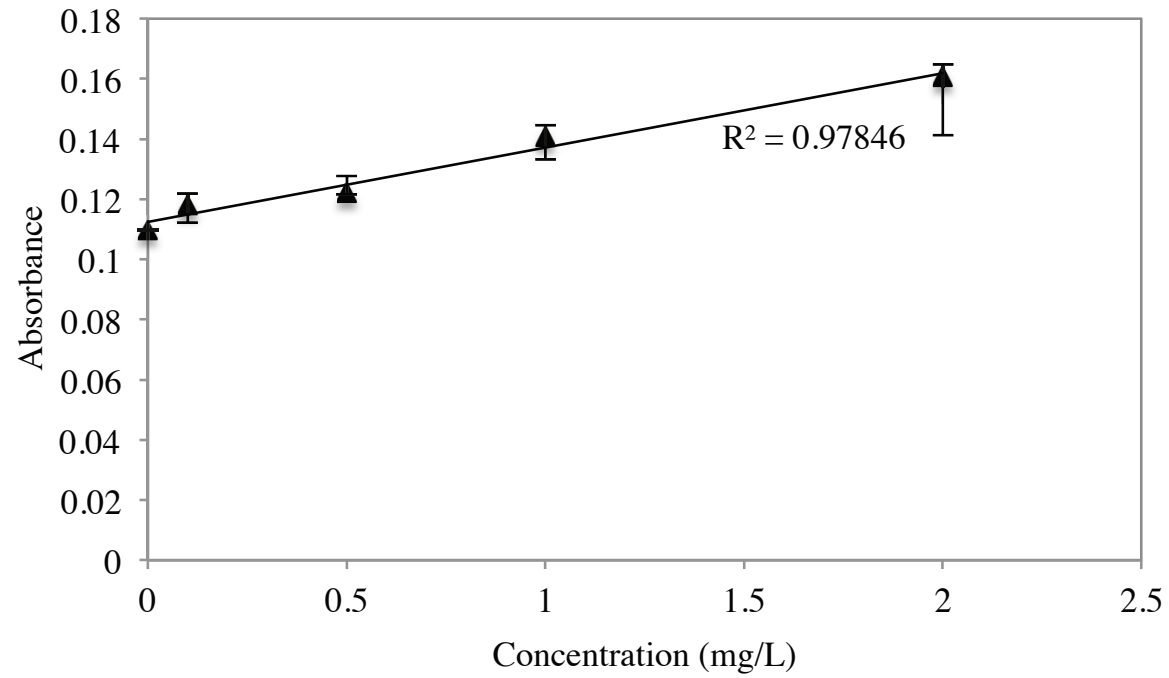

Figure 4.6 Absorbance values of Zetag8160 in DW at $\lambda=190 \mathrm{~nm}$ using the absorbance method (average of three standards) 


\subsubsection{Detection of Polymer in Rideau River Water}

Figure 4.7 demonstrates the absorbance spectra of different Zetag8160 doses in river water over wavelengths of 200-800 $\mathrm{nm}$ using the dye method. The peak absorbance values at $\lambda=646 \mathrm{~nm}$ versus polymer doses for the dye method are provided in Figure 4.8. The corresponding figures for N-Bromination method (at $200 \mathrm{~nm}<\lambda<800 \mathrm{~nm}$ with peak of absorbance at $580 \mathrm{~nm}$ ), and absorbance method (at $190 \mathrm{~nm}<\lambda<250 \mathrm{~nm}$ with peak of absorbance at $190 \mathrm{~nm}$ ) are provided in Figures 4.9 through 4.12 .

The MDL for dye, N-Bromination, and absorbance methods, calculated using Hubaux, and Vos's method are 1.1, 1.1, and $0.2 \mathrm{mg} / \mathrm{L}$ respectively. This shows that the $\mathrm{UV}-\mathrm{V}$ is method was again more sensitive than the other two methods in river water. The dye and N-Bromination methods appeared to be equally sensitive in river water. Interestingly, the detection limit of the UV-Vis absorbance method did not increase in river water in spite of the dissolved organic matter in river water. This can be explained by the peak absorbance of organic matter at $254 \mathrm{~nm}$, which is outside of the wavelength range used for polymer detection. 


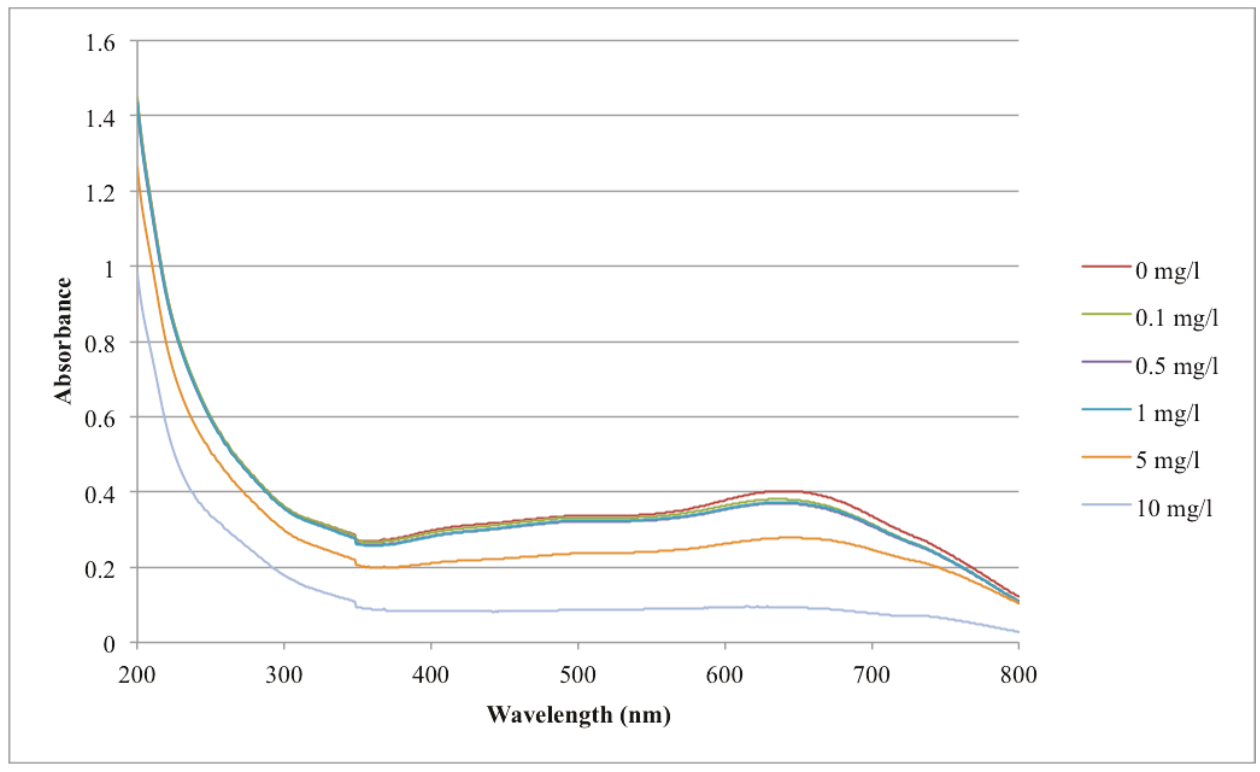

Figure 4.7 Absorbance values of different Zetag8160 concentrations in river water from $\lambda=190$ to $800 \mathrm{~nm}$ using the dye method (average of three measurements)

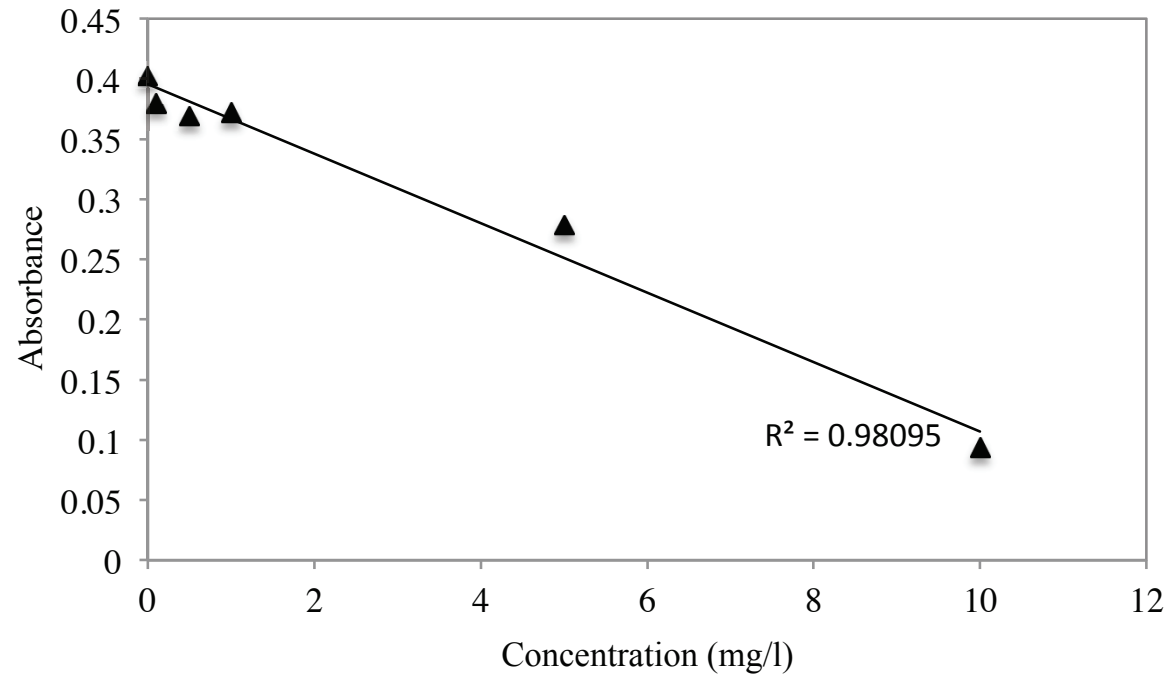

Figure 4.8 Absorbance values of Zetag8160 in river water at $\lambda=646 \mathrm{~nm}$ using the dye method (average of three measurements) 


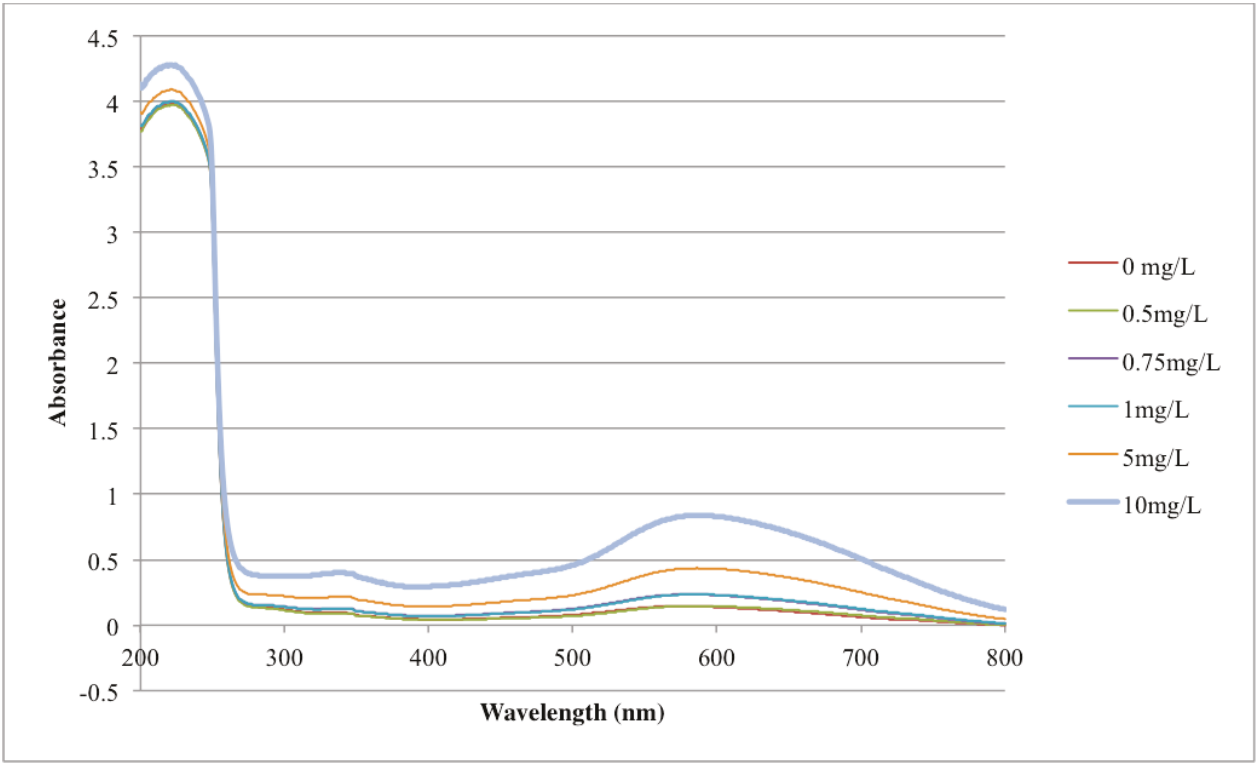

Figure 4.9 Absorbance values of different Zetag8160 concentrations in river water from $\lambda=200$ to $800 \mathrm{~nm}$ using the $\mathrm{N}$-Bromination method (average of three measurements)

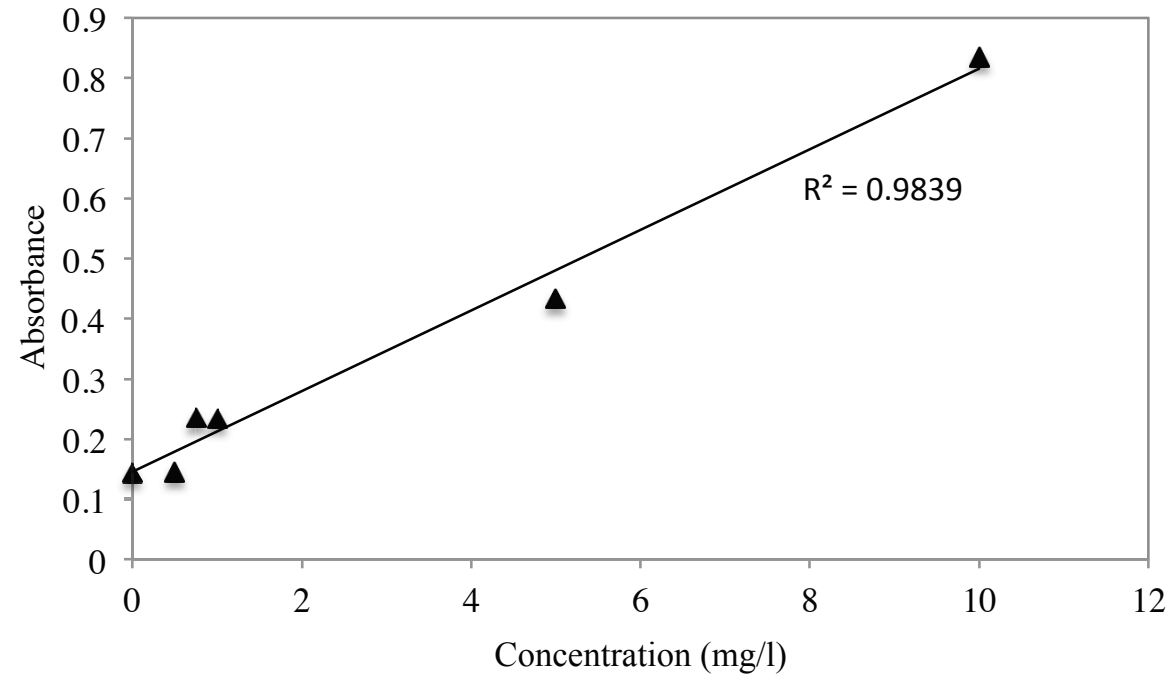

Figure 4.10 Absorbance values of Zetag8160 in river water at $\lambda=580 \mathrm{~nm}$ using the $\mathrm{N}$-Bromination method (average of three measurements) 


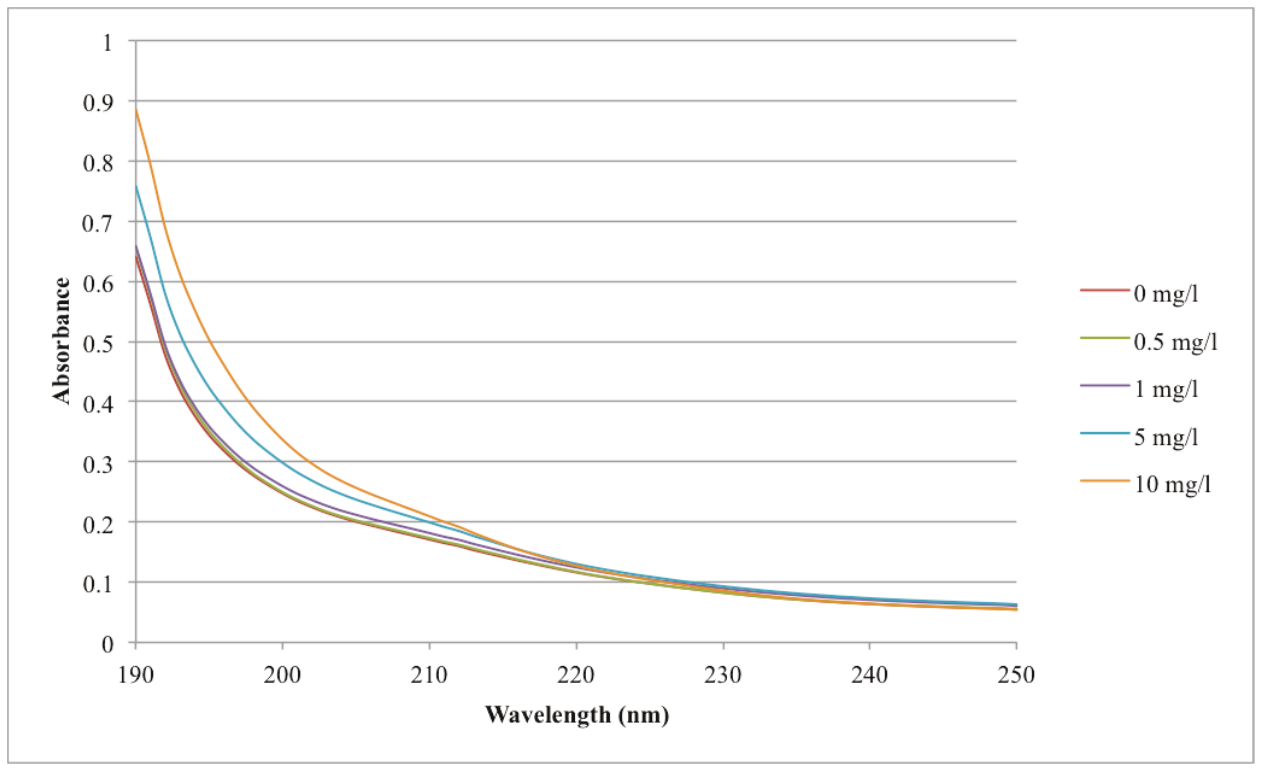

Figure 4.11 Absorbance values of different Zetag8160 concentrations in river water from $\lambda=190$ to $250 \mathrm{~nm}$ using the absorbance method (average of three measurements)

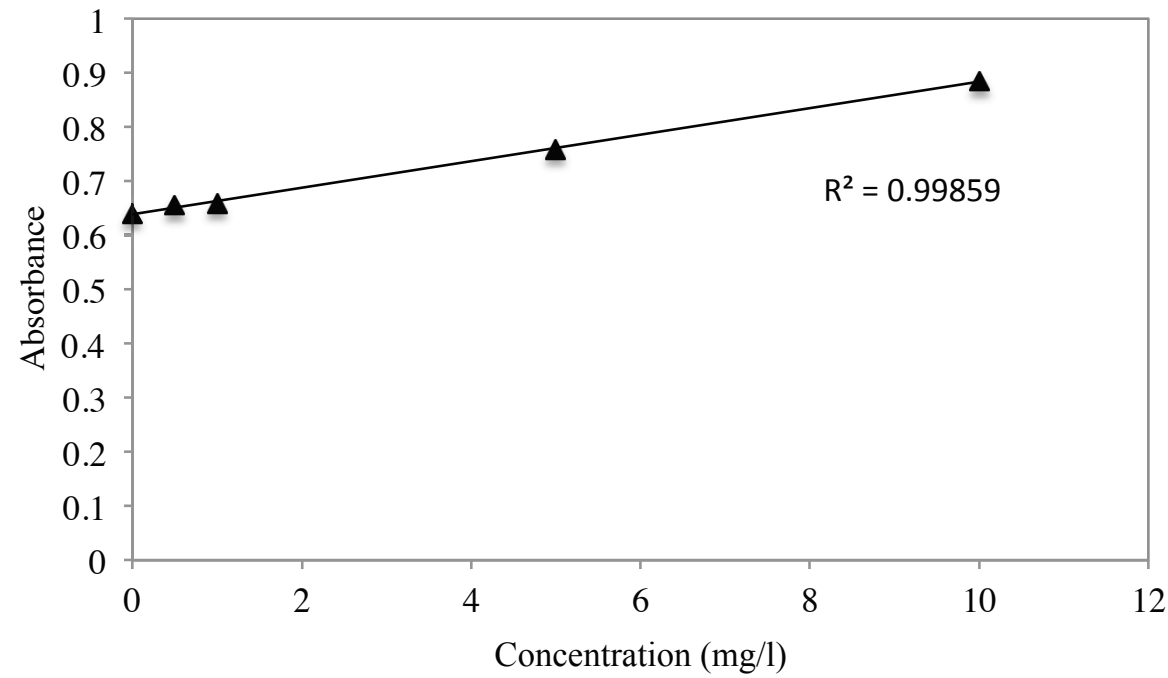

Figure 4.12 Absorbance values of Zetag8160 in river water at $\lambda=190 \mathrm{~nm}$ using the absorbance method (average of three measurements) 


\subsubsection{Detection of Polymer in Secondary Wastewater}

Figure 4.13 demonstrates the absorbance spectra of different Zetag8160 doses in secondary wastewater over wavelengths of 200-800 nm using the dye method. The peak absorbance values at $\lambda=646 \mathrm{~nm}$ versus polymer doses for the dye method are provided in Figure 4.14. The corresponding figures for N-Bromination method (at $200 \mathrm{~nm}<\lambda<800$ $\mathrm{nm}$ with peak of absorbance at $580 \mathrm{~nm}$ ), and absorbance method (at $190 \mathrm{~nm}<\lambda<250 \mathrm{~nm}$ with peak of absorbance at $190 \mathrm{~nm}$ ) are provided in Figures 4.15 through 4.18.

The MDL for dye, N-Bromination, and absorbance methods, calculated using Hubaux, and Vos's method are 1.8, 1.14, and $0.3 \mathrm{mg} / \mathrm{L}$ respectively. As before, the lowest detection limit is associated with the absorbance method indicating that it can detect lower concentrations of polymers in wastewater samples compared to the other two methods.

The MDL in wastewater for the absorbance method did not increase significantly compared to its MDL in deionized water $(0.2 \mathrm{mg} / \mathrm{L})$, and river water $(0.2 \mathrm{mg} / \mathrm{L})$. The detection limits of dye and N-Bromination methods, however, increased in wastewater samples compared to river water samples. 


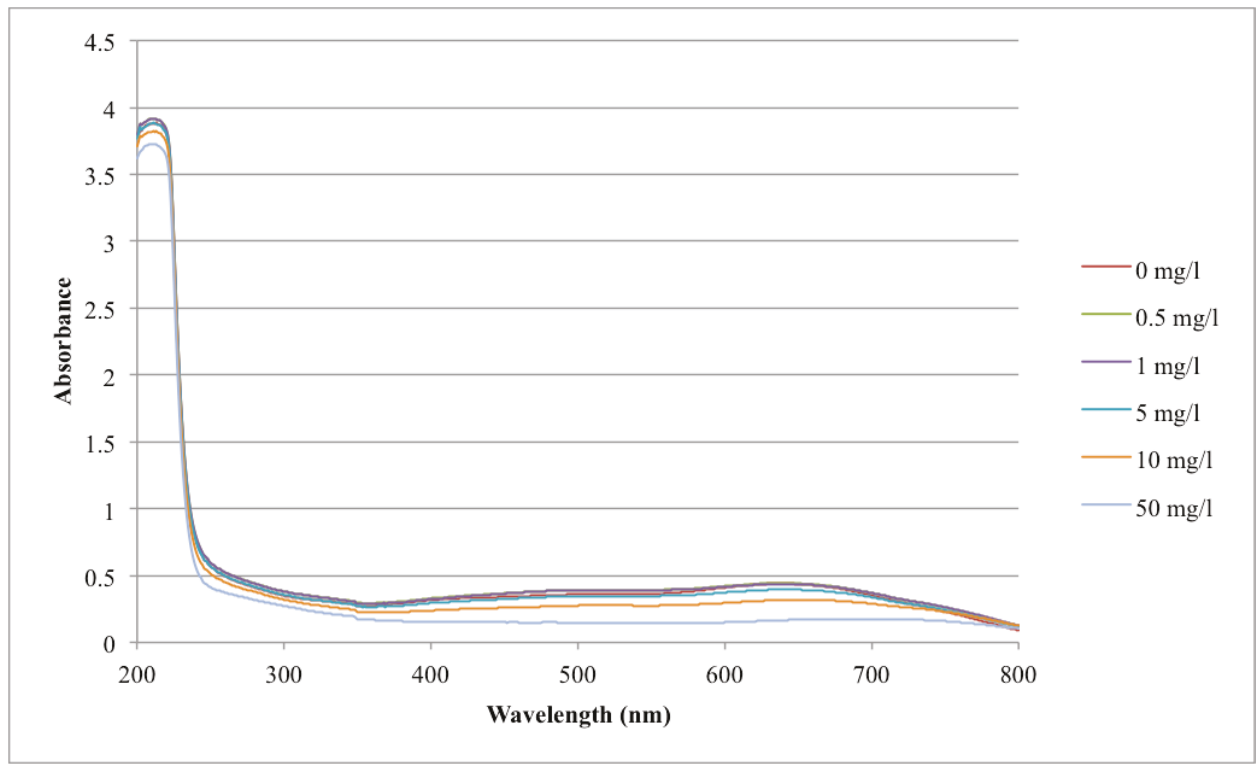

Figure 4.13 Absorbance values of different Zetag8160 concentrations in wastewater from $\lambda=190$ to $800 \mathrm{~nm}$ using the dye method (average of three measurements)

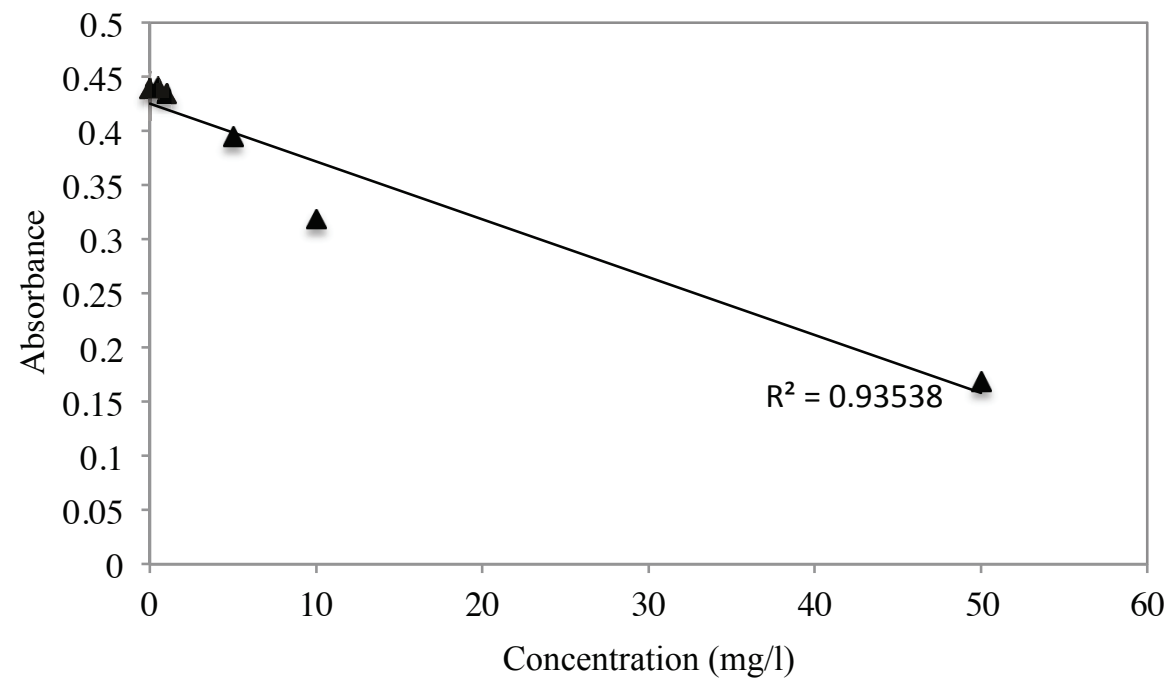

Figure 4.14 Absorbance values of Zetag8160 in wastewater at $\lambda=646 \mathrm{~nm}$ using the dye method (average of three measurements) 


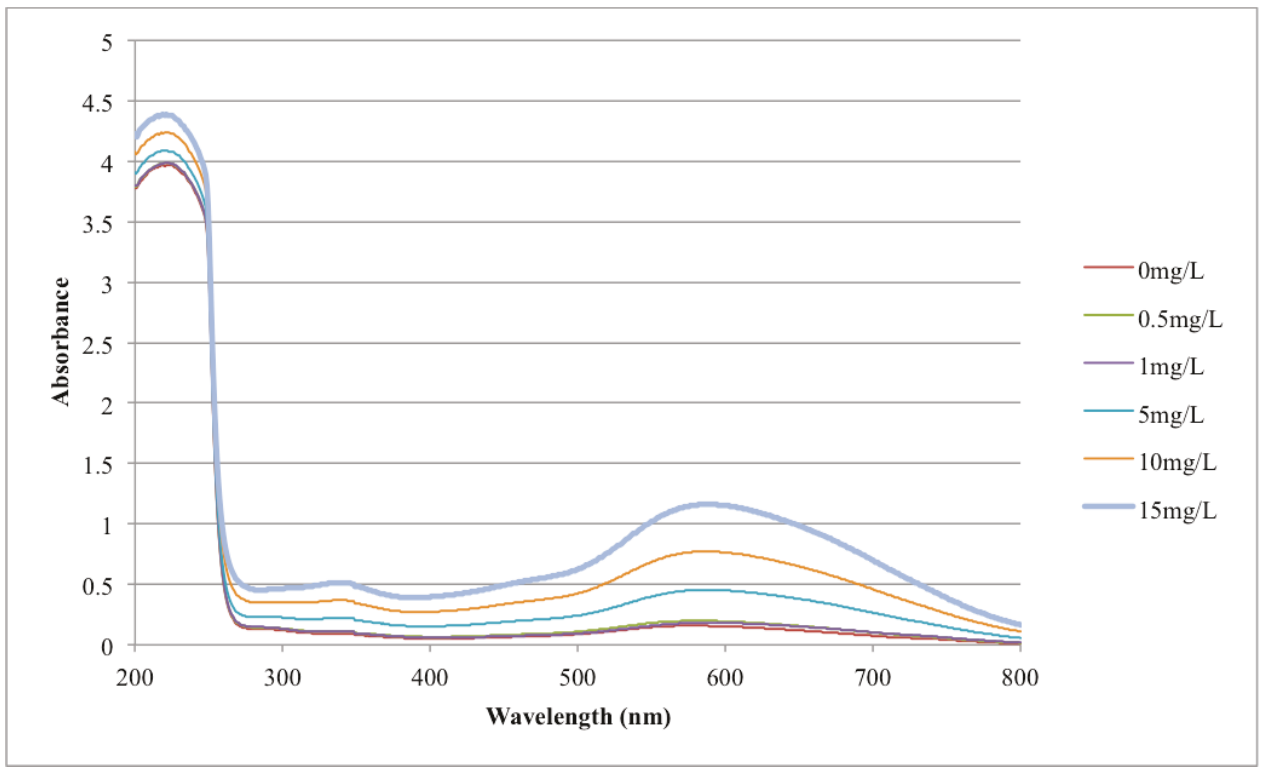

Figure 4.15 Absorbance values of different Zetag8160 concentrations in wastewater from $\lambda=200$ to $800 \mathrm{~nm}$ using the $\mathrm{N}$-Bromination method (average of three measurements)

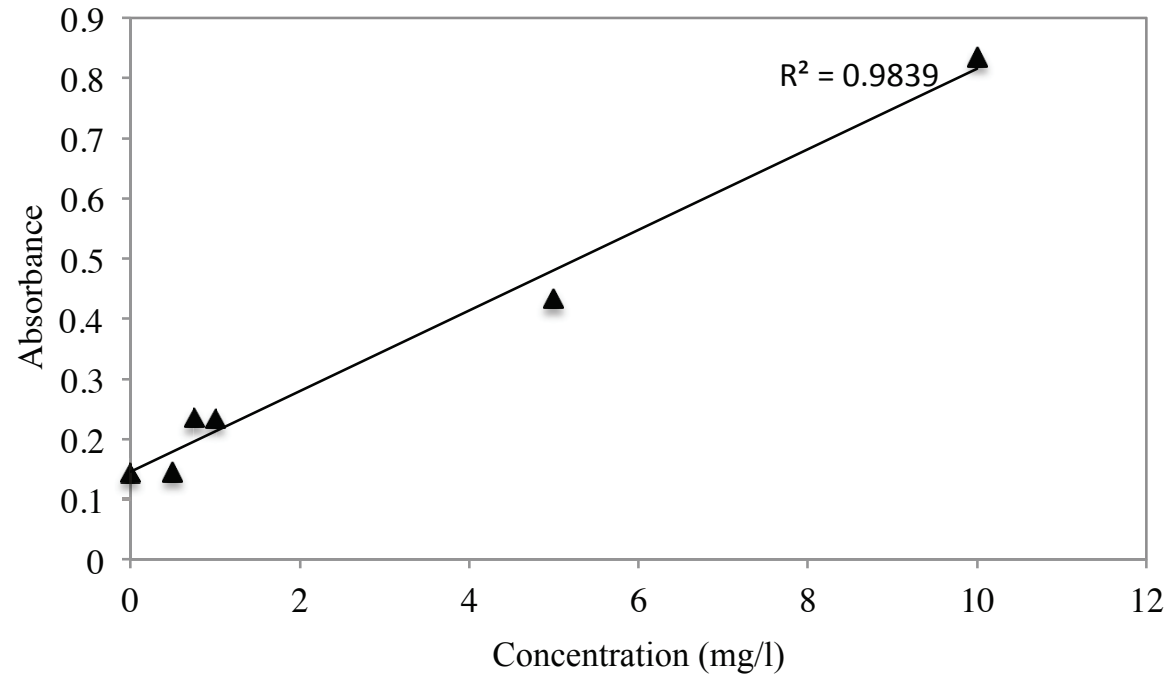

Figure 4.16 Absorbance values of Zetag8160 in wastewater at $\lambda=580 \mathrm{~nm}$ using the $\mathrm{N}$-Bromination method (average of three measurements) 


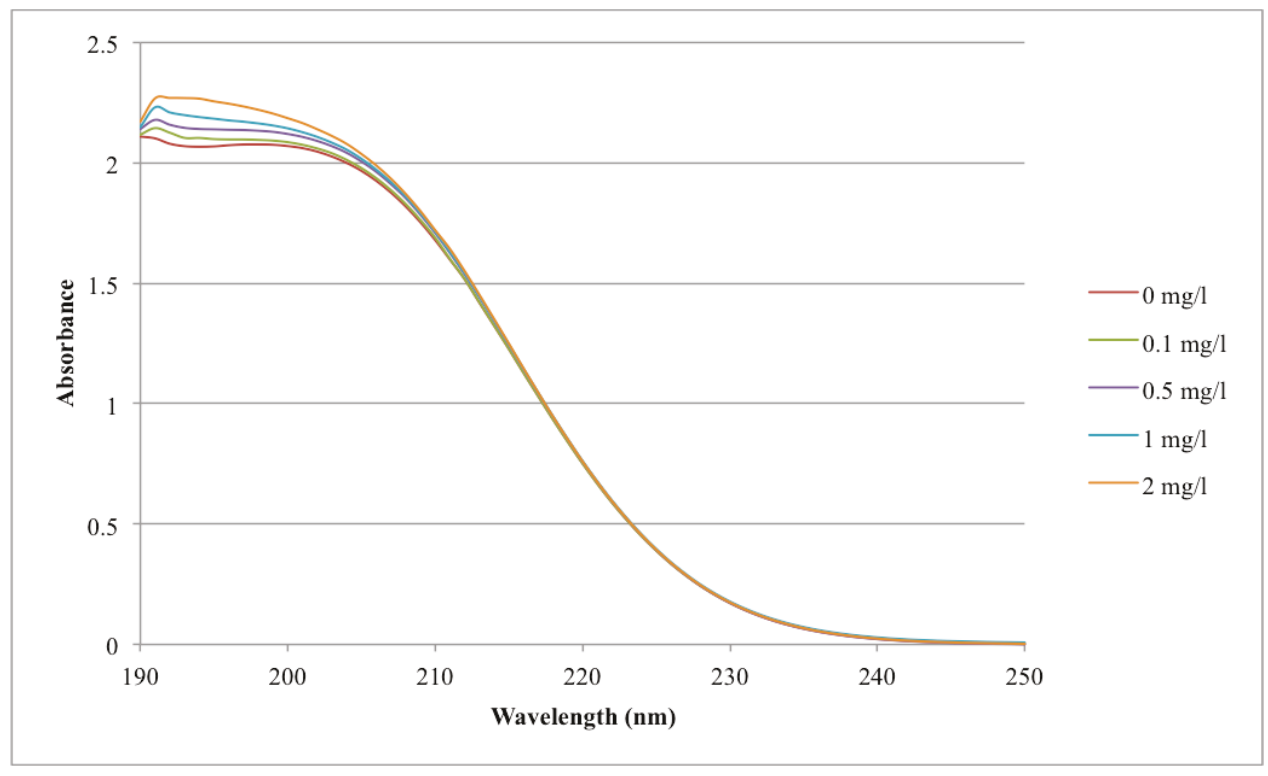

Figure 4.17 Absorbance values of different Zetag8160 concentrations in wastewater from $\lambda=190$ to $250 \mathrm{~nm}$ using the absorbance method (average of three measurements)

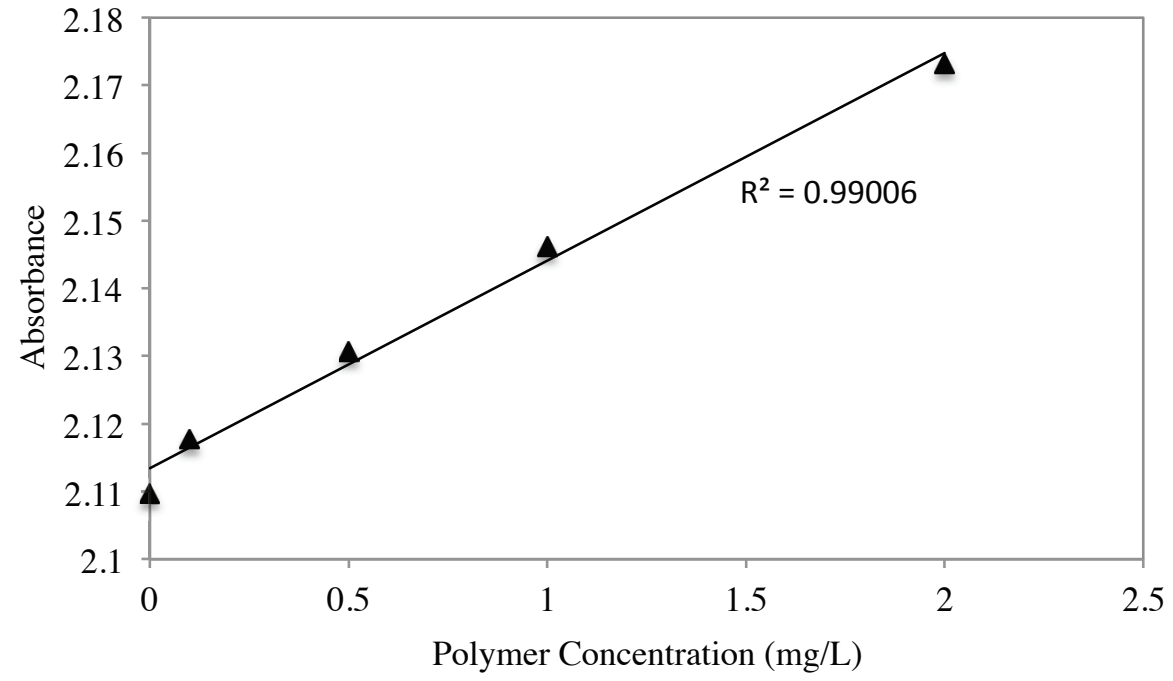

Figure 4.18 Absorbance values of Zetag8160 in wastewater at $\lambda=190 \mathrm{~nm}$ using the absorbance method (average of three measurements) 


\subsubsection{Comparison of Methods}

For ease of comparison between the method detection limits of polymer in different water matrices, all calculated MDL values associated with each method are tabulated in Table 4.1 below.

Based on MDL values provided in Table 4.1, the absorbance method has not only proved to be more sensitive in all environmental samples, but also had a very quick and safe procedure to perform, not to mention that it was inexpensive and did not require any chemicals or additional steps. The time it takes to prepare one sample to be measured via absorbance method is about 1 minute whereas for N-Bromination, and dye methods it is about 17 minutes, and 24 hours respectively due to the wait time associated with those methods. The absorbance method does not require any chemicals aside from the polymer, whereas, the dye method requires anionic salts, which cost about $\$ 100$ per $250 \mathrm{mg}$ of the salt. N-Bromination method requires bromine, cadmium iodide, sodium acetate/acetic acid buffer, and sodium formate which are also costly, not to mention the corrosivity of bromine and toxicity of cadmium iodide, which require all steps of this method to be carried out in a fume hood.

Another difficulty with the N-Bromination method was that each and every step of sample preparation were to be performed in a timely manner so that enough time was allocated for the chemicals to react, and the probability of occurrence of side reactions were minimized. This issue was not of concern in neither absorbance, nor the dye method. 
Table 4.1 Values of MDL for Zetag8160 polymer in different water samples

\begin{tabular}{l|ccc}
\cline { 2 - 4 } & \multicolumn{3}{c}{ MDL $(\mathbf{m g} / \mathbf{L})$} \\
\hline Water Matric & Dye & N-Bromination & Absorbance \\
Deionized Water & 0.6 & 0.5 & 0.2 \\
Rideau River Water & 1.1 & 1.1 & 0.2 \\
Secondary Wastewater (ROPEC) & 1.8 & 1.14 & 0.3 \\
\hline
\end{tabular}

\subsection{Conclusion}

The detection limit of Zetag8160 polymer in deionized water, Rideau River water, and secondary wastewater was calculated for the dye, N-Bromination, and absorbance methods. The absorbance method proved to be more sensitive compared to the other two methods in deionized water, as well as in river water and secondary effluent wastewater. Furthermore, the detection limit of absorbance method was consistent and did not differ much between the different environmental samples, whereas the $\mathrm{N}$-Bromination and the dye methods were affected by other constituents and dissolved organic matter, and showed greater detection limits as DOM concentration was increased in the samples. 


\section{Chapter 5: Determination of Optimum}

\section{Polymer Dose for Sludge Dewatering}

\section{Based on Residual Polymer}

\section{Concentration}

\subsection{Introduction}

The ease at which digested sludge is dewatered is mainly a function of polymer dose during conditioning phase; there is an optimum polymer dose that results in the most water release from sludge particles, above and below which dewatering is worsened. Dewatering performance also changes by variations in factors such as duration and intensity of mixing. If mixing is kept the same, polymer concentration would be the only variable to change dewatering performance.

To determine the optimum polymer dose for dewatering of digested sludge, jar testing is used. The dewaterability of the samples from the jar tester is then determined by performing tests such as Capillary Suction Time (CST), and Specific Resistance to Filtration (SRF), that typically measure ease of water release from sludge solids (Vesilind 1988). Other tests use the Cake Solids Concentration (CSC) and Filtrate Volume (FV) of jar tested samples to determine optimum polymer dosage.

Due to the continuous changes in the wastewater characteristics and related treatment processes, digested sludge characteristics also change on a continuous basis. 
Changes in digested sludge characteristics require frequent adjustments to the optimum polymer dose that results in the most water release from digested sludge. To ensure that the polymer dosed to digested sludge is at the optimum dose, jar test experiments need to be performed by the treatment plant personnel.

The difficulty with such tests is that they do not quantify a fundamentally based physical parameter of digested sludge, nor they can be easily employed for dewatering optimization, and predict the efficiency of a full-scale dewatering device (Dentel 1997). Moreover, performing such tests, requires involvement of a highly skilled operator, and fails to produce real-time results; as they are time-consuming, and by the time the results are available, they might not be applicable, due to the constant changes in digested sludge characteristics. There is therefore the need for methods that can measure optimum polymer dose in-line, and make corresponding adjustments to concentration of polymer being added to digested sludge in real-time.

Campbell and Crescuolo (1985) proposed a method for controlled addition of polymer to sludge that was based on rheological characteristics of conditioned sludge. They showed that under-conditioned sludge exhibited a relatively smooth increase in shear stress upon increase in shear rate. The increase in shear stress varied from zero, up to a value at which turbulence occurred, beyond turbulence point, further increase in shear rate resulted in a sudden increase in slope of the curve (Campbell and Crescuolo 1985). In the optimum polymer dose and consequently the over-dosing region, the behavior of sludge shear stress was different. It was found that at low shear rates, sludge exhibited a rapid increase in shear stress, which was then reversed over a subsequent shear rate period resulting in a peak (maximum). Upon further increase in shear rate, 
shear stress increased (resulting in a minimum) up to a turbulence point, after which, shear stress increased with a sudden increase in slope upon further increase in shear rate. Further increases in polymer dosage progressively increased the peak value without affecting its general positioning on the curve. It was then found that the appearance of this characteristic peak, with the slope of the curve passing through zero, corresponded closely to the optimum polymer dosage (Campbell and Crescuolo 1985).

In-line and real-time methods would be further practical if they could directly measure the concentration of residual polymer in filtrate/centrate and use that information towards adjusting the concentration of polymer being added to digested sludge. However, monitoring the concentration of polymers in the filtrate is a formidable task not well suited to industrial applications as analytical techniques for this purpose are complicated and time consuming and do not permit real-time results. Electronic instrumentation to determine electric charge is available, but such devices are expensive, and do not differentiate between charge associated with a polymer, or charge from other sources, including the water, solids, or other constituents in the effluent (Budd, et al. 1995).

Nevertheless, Budd et al. (1995) developed a new method or controlled addition of polymer in water treatment plants based on direct measurements of residual polymer concentration to produce water with low solids contents for utilization in industry. For this purpose, depending on the concentration of polymer being added to untreated water, a fluorescing molecule, with an opposite charge to that of polymer was also added. The oppositely charged polymer and fluorescing molecule then produced a complex that could be used to monitor the concentration of polymer in the effluent. The concentration of polymer being added to water could then be adjusted based on the concentration of 
polymer-fluorescing complex in the effluent using a control loop. Budd et al.'s (1995) method was the first attempt to directly measure the residual polymer concentration. However, the need for introduction of an external fluorescing molecule to water and potential consumption of the fluorescing molecule with other water constituents limited the application of the method.

Based on the successful results obtained in previous chapters, UV-Vis spectroscopy emerged as a potential method for the detection and optimization of polymers during sludge dewatering. The goal of this chapter was to determine whether the UV-Vis method could successfully be used for the detection of residual polymer in digested sludge centrate or filtrate after dewatering, and whether a relationship could be established between the residual polymer concentration in sludge centrate/filtrate and the optimum polymer dose. If successful, the UV-Vis method could potentially be used inline and real-time at treatment plants in the future to achieve optimization of polymer dose and sludge dewatering. UV-Vis spectroscopy is especially unique for the purposes of this chapter, as there exist in-line spectrophotometers that do not require contribution from wastewater treatment plant operators.

\subsection{Materials and Methods}

\subsubsection{Polymer Stock Solution}

Polymer stock solutions of $5 \mathrm{~g} / \mathrm{L}$ or $0.5 \%$ were prepared as follows: in three plastic weighing dishes, $5 \mathrm{~g}$ of Zetag8160 (polyacrylamide, medium-high cationic charge, high molecular weight, granular solid form), $5 \mathrm{~g}$ of SNF475 (polyacrylamide, high charge density, ultra-high molecular weight, granular solid form), and $5 \mathrm{~g}$ of CIBA (polyacrylamide, high charge density, high molecular weight, free-flowing microbead) 
were weighted using a scale (SI-114, Denver Instruments, Canada), and decanted into three $1 \mathrm{~L}$ glass beakers. The beakers were then diluted with deionized water up to a volume of $1 \mathrm{~L}$. Using a jar tester (Phipps and Bird, USA), the mixtures were then stirred at $300 \mathrm{rpm}$ for 5 minutes followed by 55 minutes at $250 \mathrm{rpm}$ and were allowed to rest for 60 minutes prior to usage.

\subsubsection{Dewatering Experiments}

Anaerobically digested sludge from Ottawa Wastewater Treatment Plant (with SRT of 20 days and blending ratio of $60-40 \%$ between primary sludge (PS) and waste activated sludge (WAS) before digestion) was collected and was mixed at $200 \mathrm{rpm}$ for two minutes and 30 seconds using a minimum of five different polymer concentrations using a jar tester. The polymers used for dewatering tests included Zetag8160, SNF475, and CIBA.

Capillary Suction Time (Type 319 multi-CST, Triton Electronics, Canada) and filtration tests were used as indicators to monitor the filterability of conditioned sludge samples. Three replicates were used for the CST test.

For filtration tests, $200 \mathrm{~mL}$ of the conditioned sludge samples was poured in the funnel of the filtration apparatus, using a medium-flow filter paper (\#P5, Fisher Scientific, Canada). A time of about 3 minutes was allowed for filtration and the final volume of the collected filtrate was recorded for each sample.

The filtrate from the filtration tests was then diluted at a ratio of $1 \mathrm{v}$ (filtrate): $9 \mathrm{v}$ (deionized water). The absorbance of the diluted samples was measured using a UV-Vis Spectrophotometer (Cary 100 Bio UV-Vis Spectrophotometer, Varian Inc./Aligant 
Technologies, Canada) and was recorded at a range of 190-300 nm using the Scan software (Cary WinUV, Varian Inc., Canada).

\subsection{Results and Discussion}

\subsubsection{Capillary Suction Time (CST)}

CST is the time the liquid fraction of the conditioned sludge takes to travel $1 \mathrm{~cm}$ radially. Once sludge is introduced into the cylinder in Figure 5.1, its liquid portion is dispersed over a filter/blotter paper underneath the cylinder. There exist two sensors located on two circles $1 \mathrm{~cm}$ apart from each other on a glass sheet beneath the filter paper. Once liquid portion hits the first sensor, a digital timer is activated which then stops once liquid gets to the second sensor.

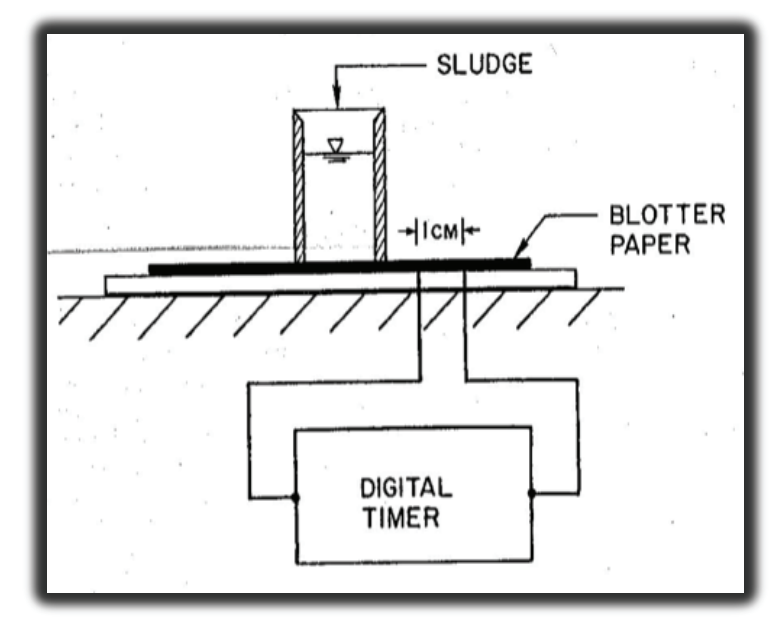

Figure 5.1 Mechanism of a CST test (adopted from Ormeci, 2013)

For the optimally conditioned sludge samples, the liquid portion takes shorter time to travel from the first circle to another. Once sludge is in the under-dose region, the compressible and non-porous sludge solids do not allow for the liquid portion to travel fast. Whereas in the over-dose region, due to the lack of available solid surfaces for interaction with polymer and the escape of polymer to the liquid phase, the liquid portion gets viscous and again takes longer time to travel from the first sensor to the other. This 
makes under/over-conditioned sludge samples to have higher CST values compared to that of optimally conditioned sludge and results in a U shape plot of CST versus polymer concentration, with the polymer dose corresponding to minimum CST value as the optimum dose.

Figures 5.2 through 5.4 demonstrate the change in CST values of anaerobically digested sludge as the dosage of conditioner is changed for the three polymers of Zetag8160, SNF475, and CIBA respectively. The expected U shape trend is clearly evident with all of the different polymers used.

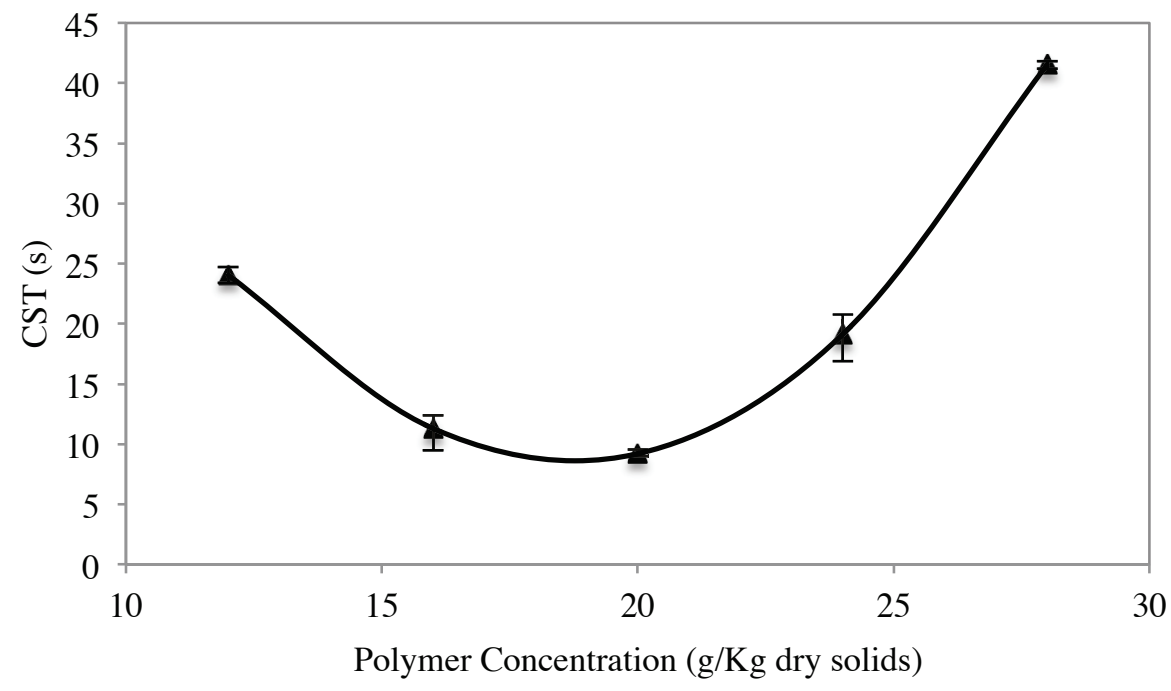

Figure 5.2 Zetag8160 CST test (average of three measurements) 


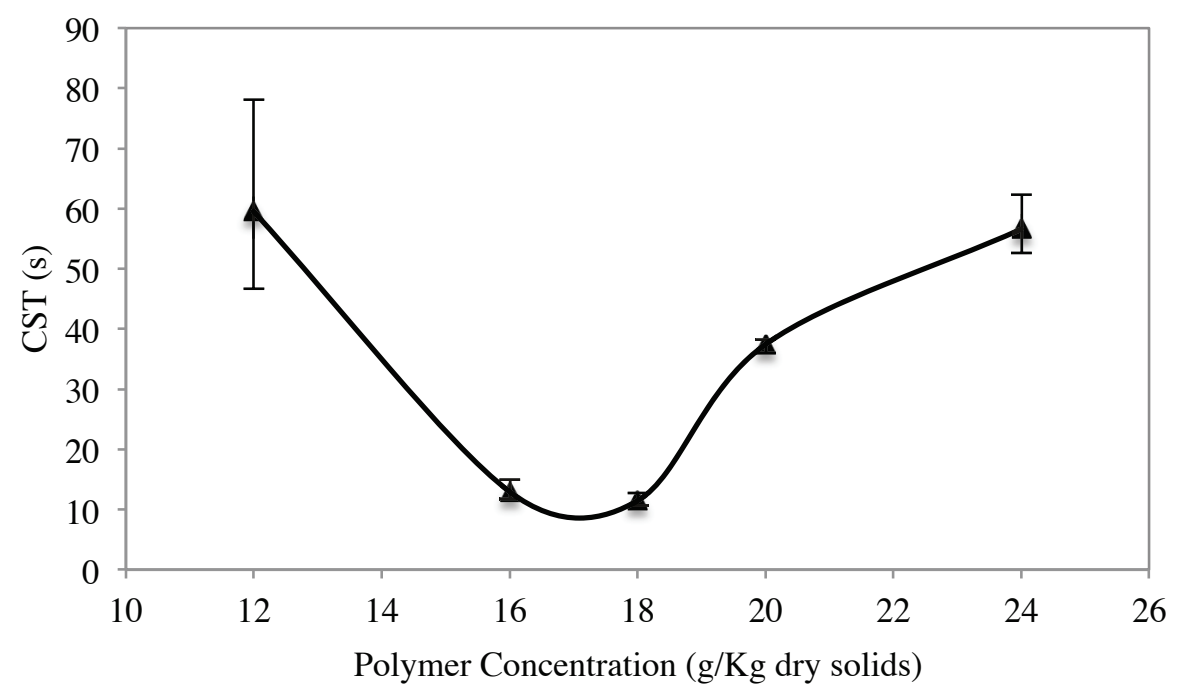

Figure 5.3 SNF475 CST test (average of three measurements)

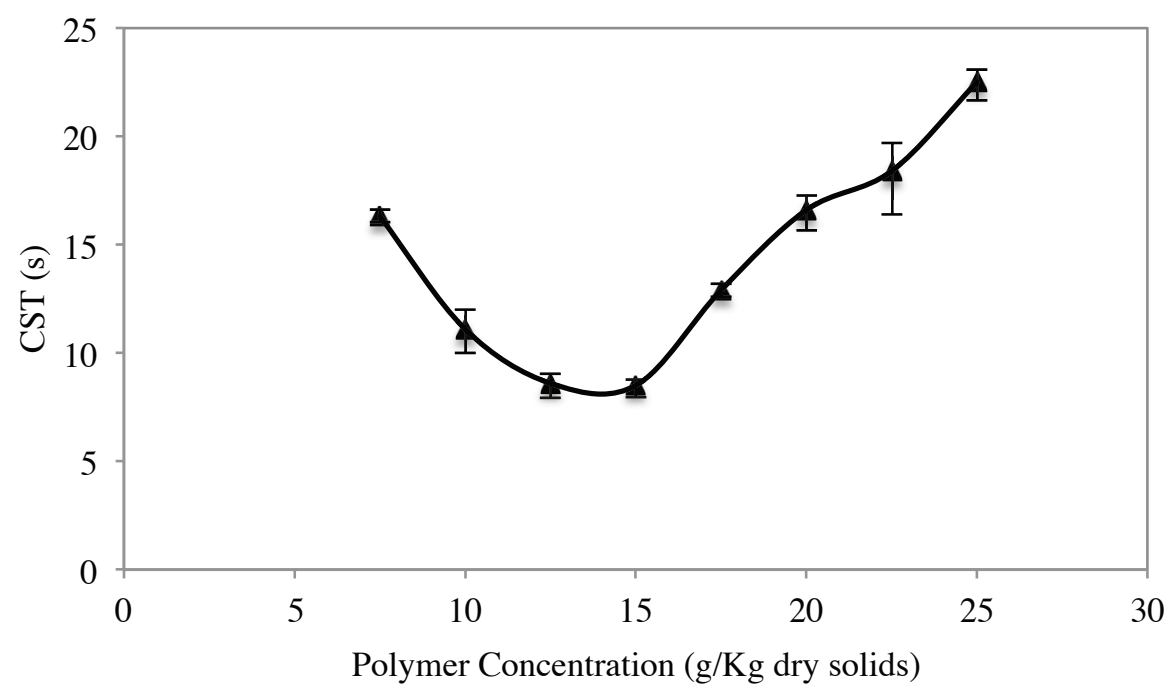

Figure 5.4 CIBA CST test (average of three measurements)

As expected, the optimum polymer dosages are different between the three different polymers varying from $15-20 \mathrm{~g}$ polymer/Kg dry solids, with CIBA having the lowest and Zetag8160 having the highest optimum dose. The differences in the polymer doses can be explained by the differences in the chemistry of polymer and their compatibility with the characteristics of the sludge used. 
Usually CST values of 20-30 seconds or lower are acceptable for the optimum dose associated with a polymer, otherwise the use of the polymer may not be recommended. Based on Figures 5.2 through 5.4 above, all the polymers used result in CST values between 8 to 11 seconds, indicating that they could all be practical for conditioning this sludge.

\subsubsection{Filtration Tests}

The apparatus for filtration test is shown in Figure 5.5 below. Conditioned sludge samples are decanted in $200 \mathrm{~mL}$ amount in the funnel at the top of the graduated cylinder. A timer goes on once the pump is turned on and dewaterability is measured in terms of amount of filtrate collected in $\mathrm{mL}$ in 3 minutes. Filtrate volume is highest at the optimum polymer dose. As polymer dose increases, an increase in the amount of filtrate is expected until the optimum point is reached after which, further increase in polymer, results in reduction in filtrate volume. This provides the shape of an upside down $\mathrm{U}$ for the plot of filtrate volume versus polymer concentration. The same reasons provided in the previous CST section as to why dewatering before and beyond optimum dose is worsened also apply here.

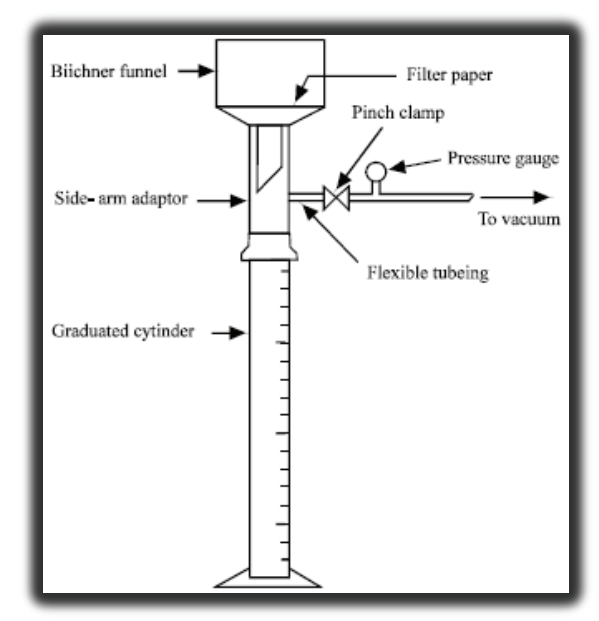

Figure 5.5 Filtration test apparatus (adopted from http://scialert.net/) 
Figures 5.6 through 5.8 show the curves for volume of filtered sludge water as concentration of polymers of Zetag8160, SNF475, and CIBA are increased.

Due to the differences in the chemistry of the polymers tested, different optimum doses would be anticipated from conditioning of sludge with each polymer. The optimum dose associated with the three polymers ranged from 17-20 g polymer/Kg dry solids with CIBA polymer having the lowest optimum dose, and Zetag8160 polymer having the highest. The same trend was observed for CST tests of the three polymers.

For the polymers of Zetag8160 and SNF475, the CST and filtration tests agree on the same polymer dose to be the optimum. For the CIBA polymer, the CST test shows the optimum dose to be $15 \mathrm{~g}$ polymer/Kg dry solids whereas in the filtration test, a sludge sample containing $17.5 \mathrm{~g}$ polymer/Kg dry solids appears to have the highest volume of filtrate produced. This is most probably due to the fact that the sub-samples taken (for CST and filtration tests) from mother-sample differed in terms of the solid/liquid content. In other words, since these samples were poured from a larger conditioned and flocculated mother sample, there is a high chance that the solid and liquid proportions were not equal for different sub-samples. Therefore, some variation in results between different sub-samples taken for CST and filtration tests could be expected. 


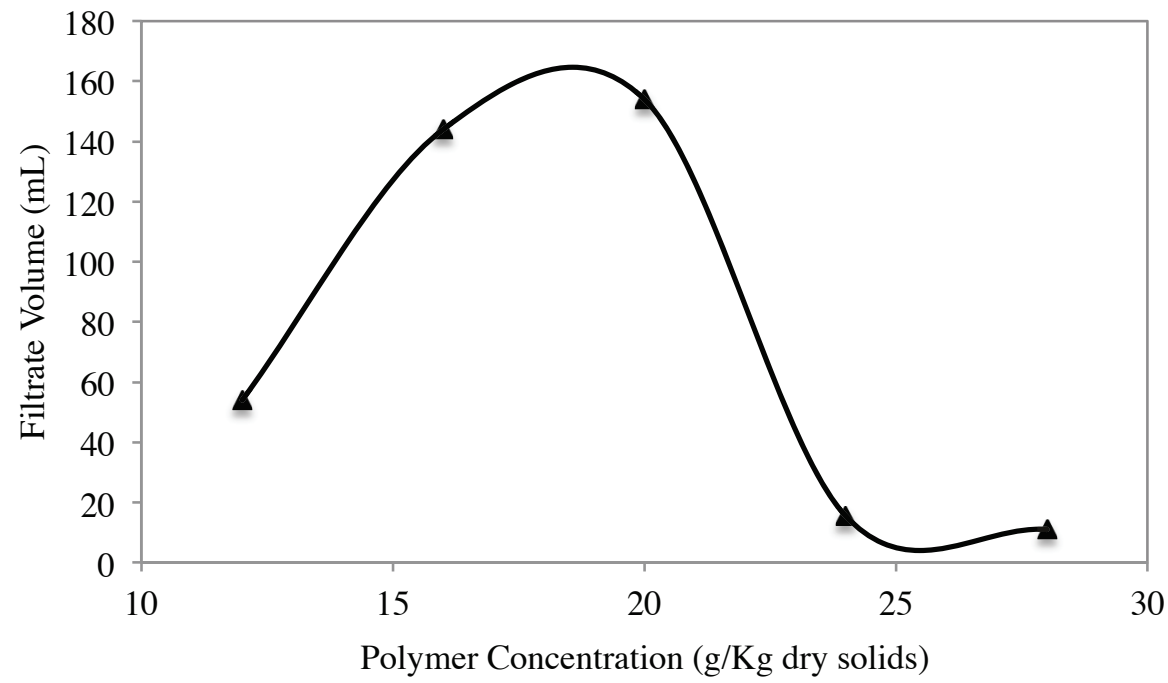

Figure 5.6 Zetag8160 filtration test

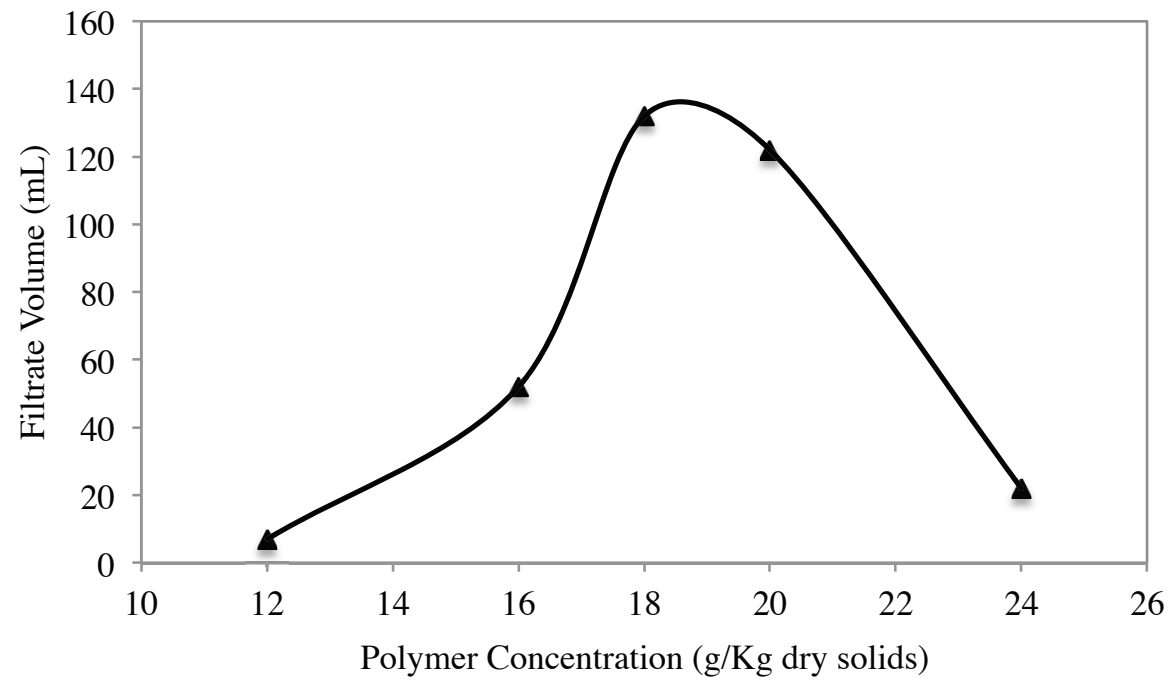

Figure 5.7 SNF475 filtration test 


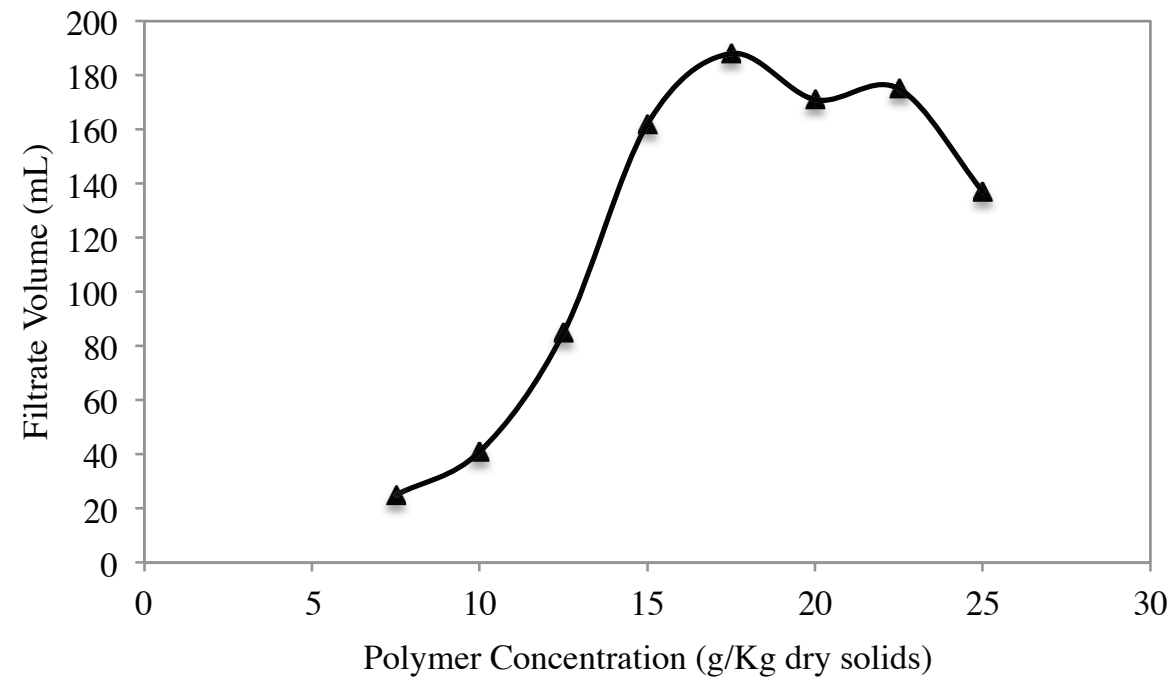

Figure 5.8 CIBA filtration test

\subsubsection{Absorbance Method}

The absorbance spectra of Zetag8160 polymer in filtrate of differently conditioned sludge samples over a wavelength of $190-800 \mathrm{~nm}$ is provided in Figure 5.9. Due to the peak of absorbance occurring at $\lambda=190 \mathrm{~nm}$, the absorbance values at $190 \mathrm{~nm}$ are plotted against polymer concentration in Figure 5.10. The same measurements for SNF475 polymer are provided in Figures 5.11 and 5.12, and for CIBA polymer in Figures 5.13 and 5.14, respectively.

As shown in Figures 5.10, 5.12, and 5.14, as the concentration of polymers are increased, the absorbance of diluted filtrate decreases up to a point, after which, further increase in polymer concentration results in an increase in the filtrate absorbance. This trend is very similar to what would be expected from a conventional filterability test such as CST.

This absorbance behavior of filtrate at $\lambda=190 \mathrm{~nm}$ could be explained by the fact that as polymer dose increases, the flocs get bigger and stronger and the probability that smaller particles get caught up in the flocs increases. As smaller particles are removed 
from filtrate and are incorporated in the flocs, the filtrate gets purer and this results in a decrease in the absorbance of filtrate. This trend continues until the optimum dose is reached. Beyond the optimum point, the extra polymer associates in the liquid (filtrate) phase directly due to the lack of new sludge surfaces to react with, and therefore increases the absorbance of the filtrate at $190 \mathrm{~nm}$. Consequently, the absorbance of the filtrate exhibits a $U$ shaped curve and the minimum absorbance corresponds to the optimum polymer dose.

In Figure 5.10, the increase in filtrate absorbance in the over-dosing region is clearly evident. However in Figures 5.12, and 5.14 this increase is not as steep as Figure 5.10 , and instead of having one point corresponding to a minimum absorbance value, the plots exhibit a minimum plateau. This could be due to the dilution ratio selected as the absorbance values are relatively on the high side. Nonetheless, the first point in the plateau (minimum region) corresponds to the optimum dose for SNF475, and CIBA polymers.

Based on the explanation given above, the optimum dose for Zetag8160, and SNF475 polymer appears to be $20 \mathrm{~g} / \mathrm{Kg}$ dry solids, whereas for CIBA polymer it is 17.5 $\mathrm{g} / \mathrm{Kg}$ dry solids. 


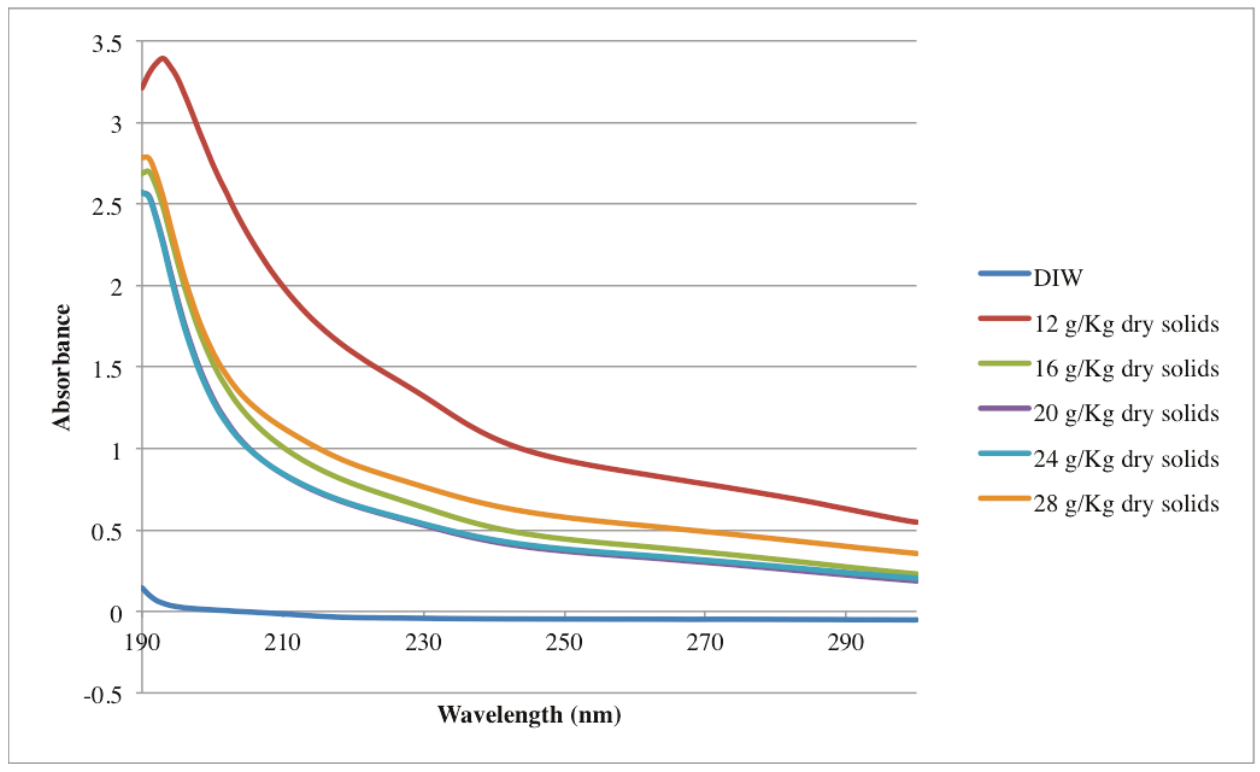

Figure 5.9 Zetag8160 absorbance after dilution at 1v(filtrate): 9v(DW) (average of three measurements)

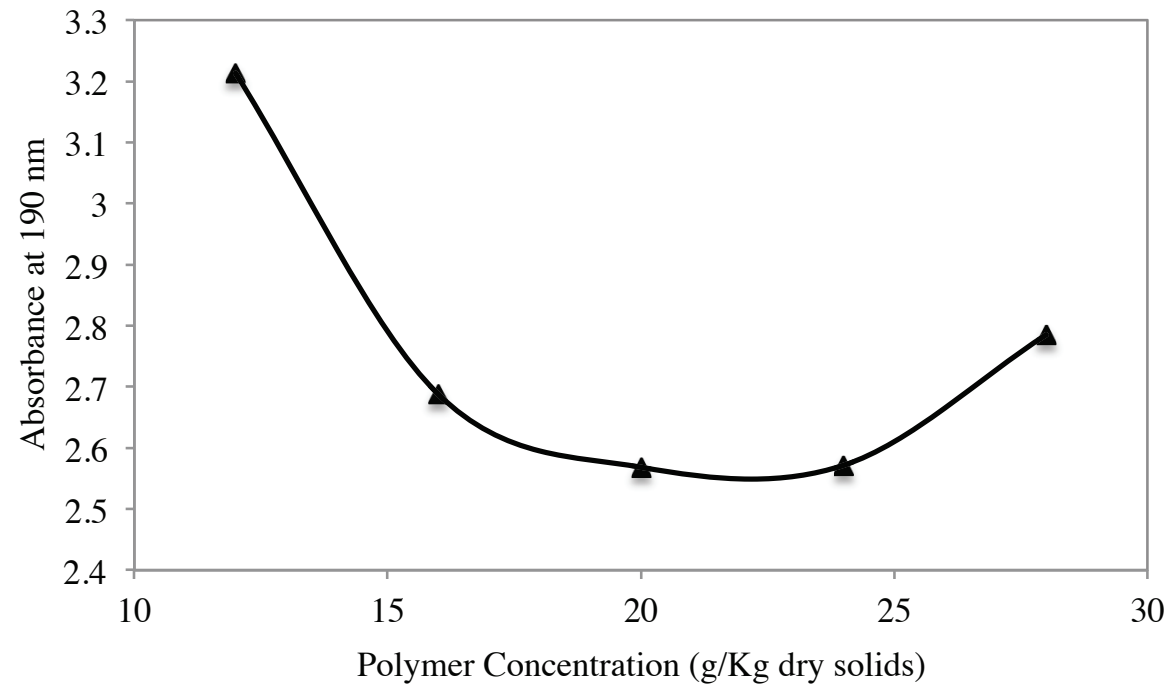

Figure 5.10 Zetag8160 absorbance test (average of three measurements) 


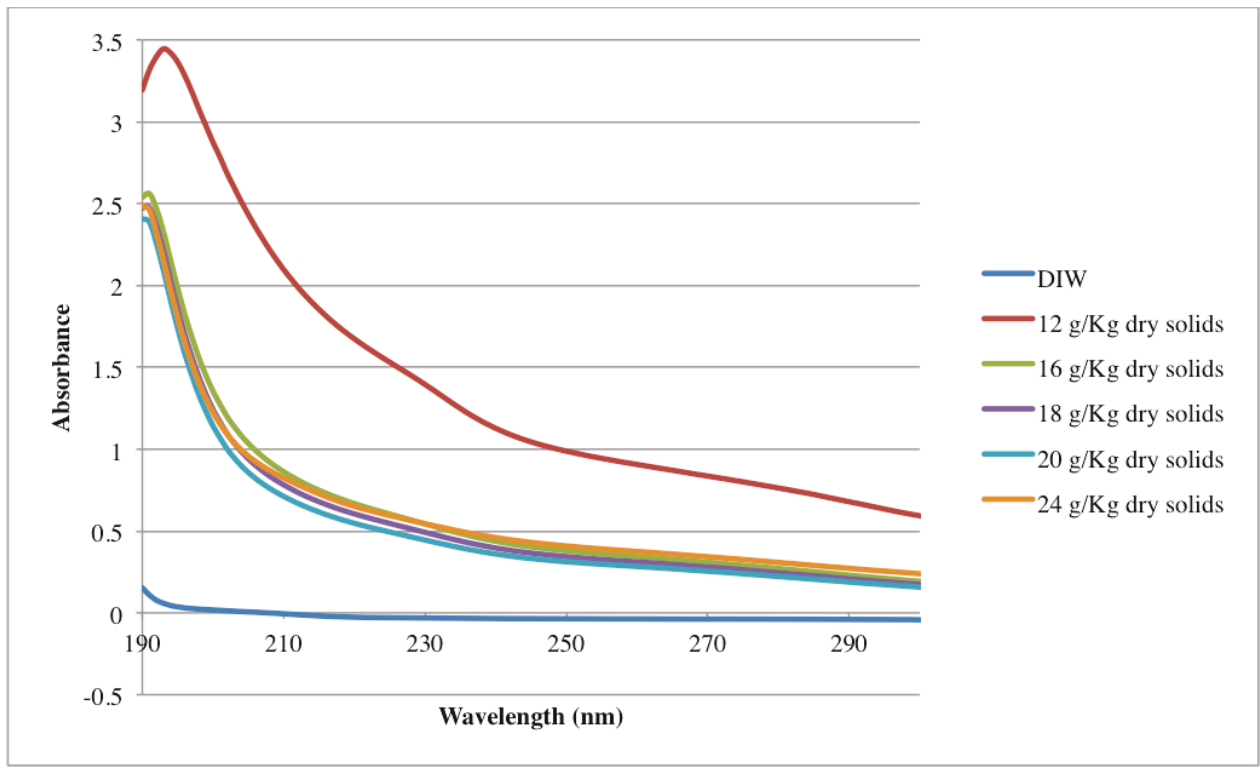

Figure 5.11 SNF475 absorbance test after dilution at 1v(filtrate): 9v(DW) (average of three measurements)

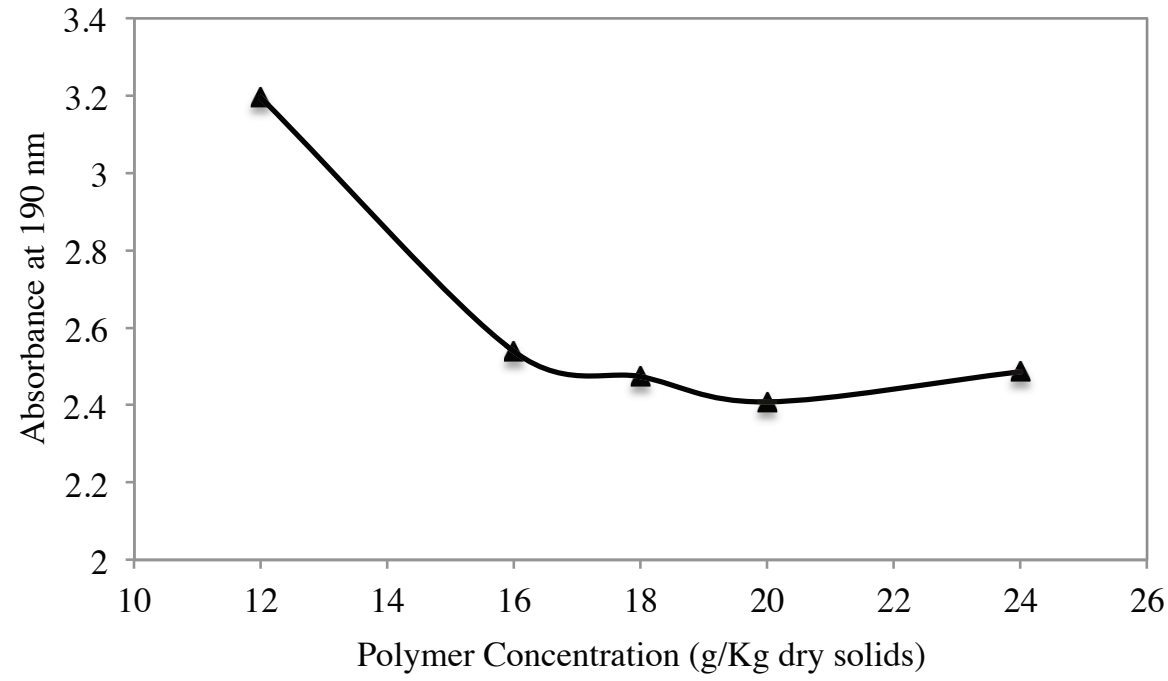

Figure 5.12 SNF475 absorbance test (average of three measurements) 


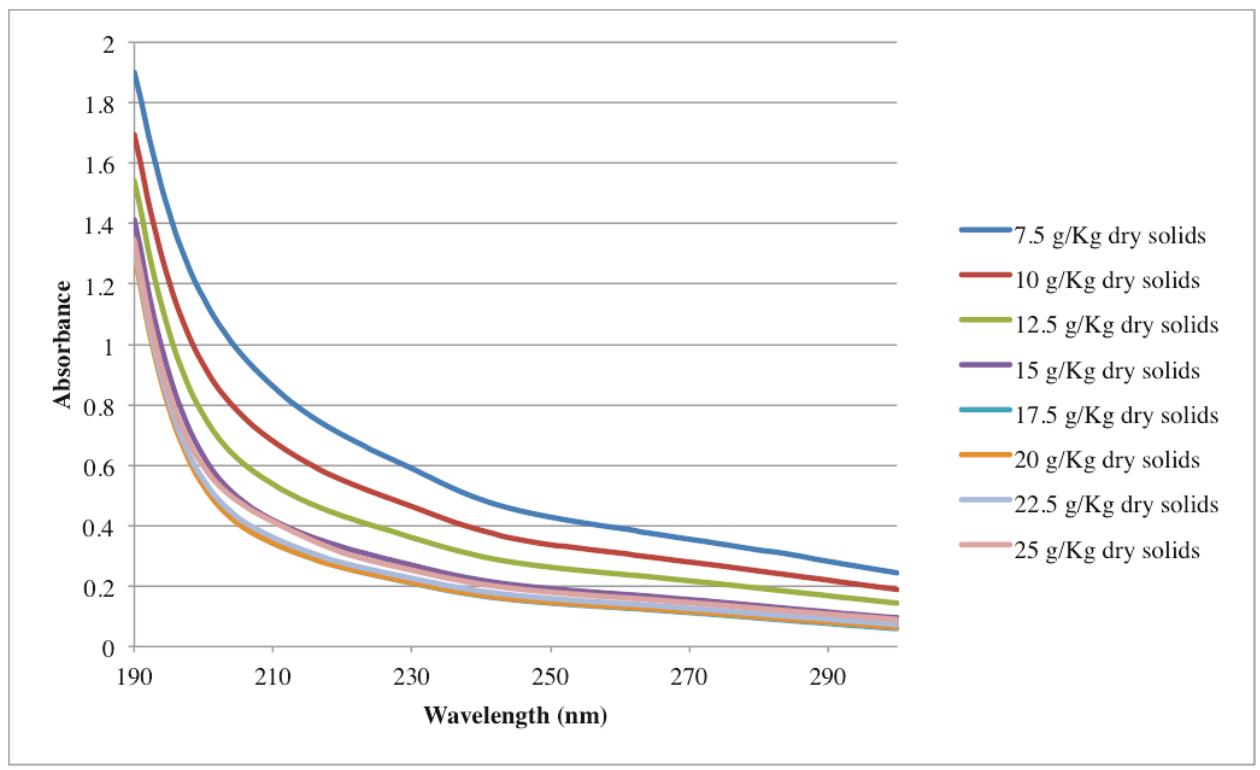

Figure 5.13 CIBA absorbance test from after dilution at $1 \mathrm{v}($ filtrate): $9 \mathrm{v}(\mathrm{DW})$ (average of three measurements)

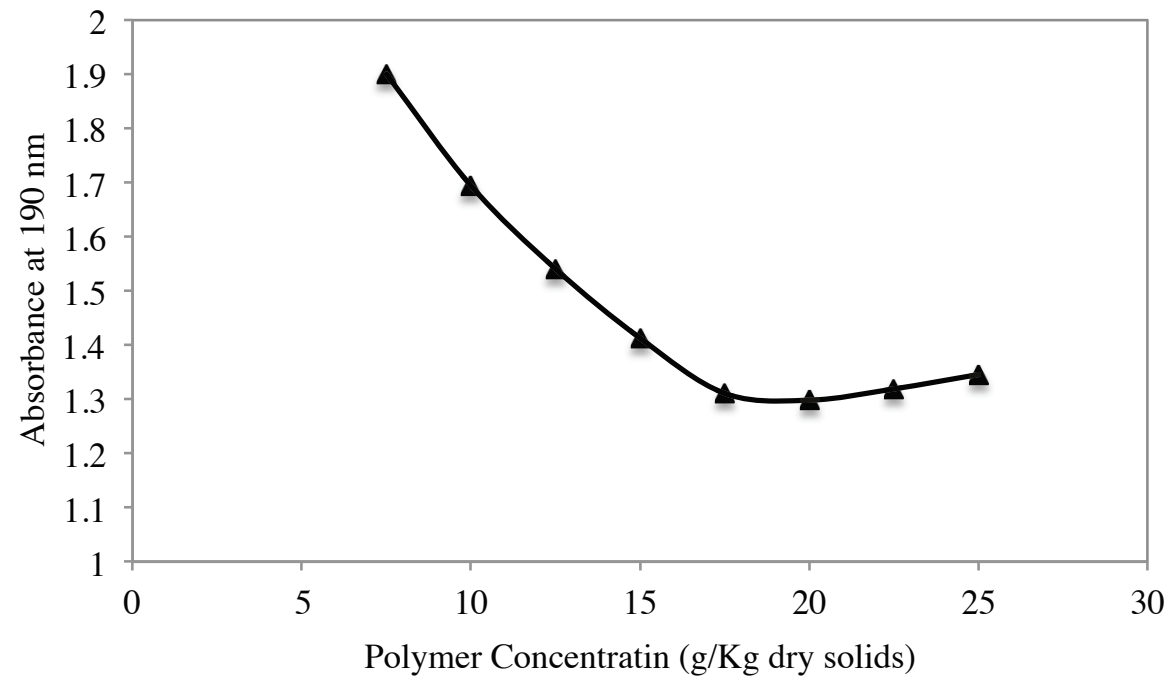

Figure 5.14 CIBA absorbance test (average of three measurements) 


\subsubsection{Comparison of Optimum Doses}

The purpose of running CST, and filtration tests in this chapter was to determine whether the optimum polymer dose specified by UV-Vis spectroscopy would be in agreement with the optimum doses determined by the conventional filterability tests. The optimum doses approximated by each method and for each polymer are provided in Table 5.1 below.

Table 5.1 Optimum doses for all polymers determined by all methods

Optimum dose for: (g/Kg dry solids)

\begin{tabular}{c|ccc}
\hline Polymers: & CST & Filtration test & Absorbance test \\
Zetag8160 & 20 & 20 & 20 \\
SNF475 & 18 & 18 & 20 \\
CIBA & 15 & 17.5 & 17.5 \\
\hline
\end{tabular}

As demonstrated in Table 5.1, for two out of the three polymers assessed, the result from absorbance method either agreed with the result of one (CIBA) or both tests (Zetag8160), and for SNF475 polymer there was a close agreement between the optimum doses determined by absorbance test to that of CST, and filtration tests. The differences could be explained by the differences in samples, different structures of polymers as well as the chosen incremental increases in polymer.

\subsubsection{Use of Absorbance Method as an In-Line Method}

The results illustrated herein indicate that the UV-Vis absorbance method can not only be used for the detection and quantification of residual polymer in filtrate or centrate 
after sludge dewatering but also for the determination of the optimum polymer dose required to condition sludge.

A control loop such as the one presented in Figure 5.15 can be used in an automated system to identify the optimum polymer dose in-line and make frequent adjustments in polymer dosage being added to sludge in real-time.

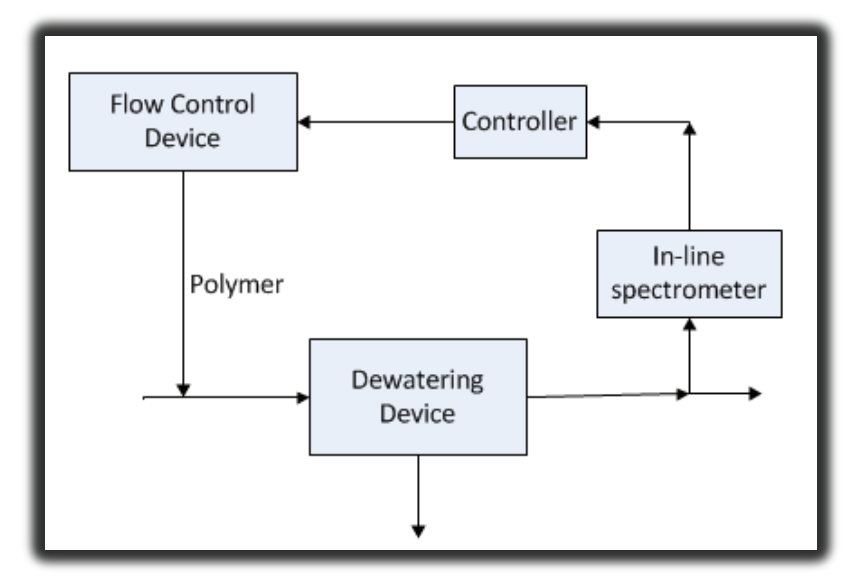

Figure 5.15 Example of a control loop for automatic adjustments in dosage of polymer being added to sludge

UV-Vis spectroscopy is especially suitable for this purpose, since there is no need for chemical reagents and also no need for an operator to be involved, since in-line spectrophotometers such as the one depicted in Figure 5.16 can also dilute the centrate with dilution water once they are programmed properly.
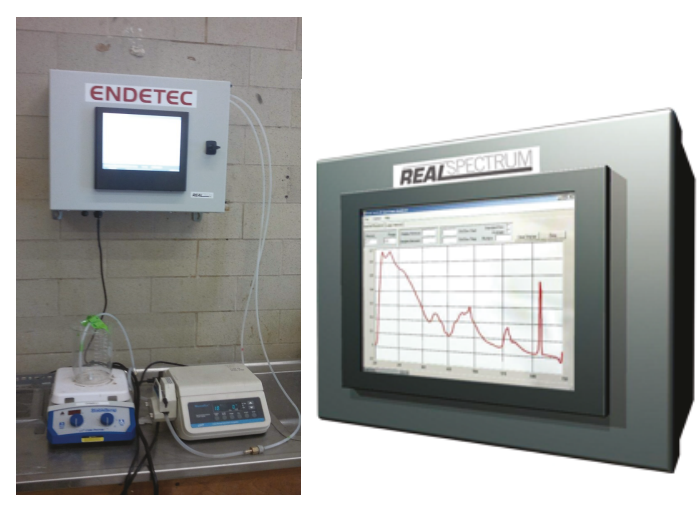

Figure 5.16 In-line UV-Vis spectrophotometers 
It should be noted however that the in-line spectrophotometer shown in Figure 5.16 was not used to measure the absorbance of any of the samples prepared for this study. Instead a benchtop spectrophotometer namely Cary 100 Bio UV-Vis Spectrophotometer from Varian Inc./Aligant Technologies was used.

\subsection{Conclusions}

A new UV-Vis based method for detection of optimum polymer dose based on absorbance of centrate exiting the dewatering unit was established for anaerobically digested sludge collected from ROPEC.

For the three different polymers assessed, the results from UV-Vis based method were in close agreement with conventional dewatering tests such as CST and filtration test.

Based on the capability of UV-Vis spectroscopy in determining optimum polymer dosage, and existence of in-line and programmable spectrophotometers, it was concluded that UV-Vis spectroscopy has the potential to be used in a control loop as an automated system for making adjustments in dosage of polymer being added to sludge based on the absorbance of centrate or filtrate exiting the dewatering unit. 


\section{Chapter 6: Conclusions}

The conclusions from this study can be listed as follows:

- A new UV-Vis based method (at a wavelength of $190 \mathrm{~nm}$ ) was established to determine the concentration of polyacrylamide polymers in deionized water. Using the Hubaux and Vos's method to calculate the detection limit for the $\mathrm{UV}-\mathrm{Vis}$ based method, a value as low as $0.2 \mathrm{ppm}$ was observed.

- The effect of $\mathrm{pH}$ on the UV-Vis based method was studied, and it was found that the method was not interfered by changes in $\mathrm{pH}$ within the range of 5-9. However, $\mathrm{pH}$ values lower than 5 and higher than 9 showed interference with the method. This was not an issue of concern for application of this method in water and wastewater treatment as water and wastewater usually have a $\mathrm{pH}$ value between 6 to 7 .

- The interferences on the UV-Vis based method were extended to $\mathrm{NaCl}, \mathrm{CaCl}_{2}$, alum, ferric chloride, and ferrous chloride. All of the chemicals showed strong absorbance between $190 \mathrm{~nm}<\lambda<200 \mathrm{~nm}$ starting at very low concentrations except alum, which did not show significant absorbance at concentrations lower than $50 \mathrm{mg} / \mathrm{L}$. It was therefore concluded that new calibration curves needed to be established once existence of such chemicals was confirmed within a sample for accurate determination and quantification of polyacrylamide. Furthermore, the results proved capability of UV-Vis spectroscopy for detection and quantification of such chemicals. 
- Choosing two sensitive methods of amide hydrolysis and N-Bromination from literature, the performances of the methods for quantification of polyacrylamide in different water matrices of deionized water, local river water, and secondary wastewater were compared to that of UV-Vis based method. The results showed that the UV-Vis based method (at $\lambda=190 \mathrm{~nm}$ ) had the lowest detection limit amongst all methods, and therefore it emerged as a successful method for detection of polyacrylamide in environmental samples in addition to deionized water.

- Finally, a relationship between the absorbance of filtrate and polymer concentration being added to sludge was established using UV-Vis spectroscopy at $\lambda=190 \mathrm{~nm}$. The constructed relationship adopted a $\mathrm{U}$ shape trend with the minimum absorbance value corresponding to optimum polymer dose. The optimum polymer dose indicated by the UV-Vis based method at $190 \mathrm{~nm}$ for different tested polymers was in close agreement with optimum polymer concentration detected by common dewatering tests of CST, and filtration. By means of in-line UV-Vis spectrophotometers, this relationship can potentially be used to create a control loop for automatic adjustments in dosage of polymer being added to sludge in real-time in the near future. Other coagulation processes can also benefit from such control loops. 


\section{Appendices}

\section{Appendix A - Supplementary Figures of Chapter 2}

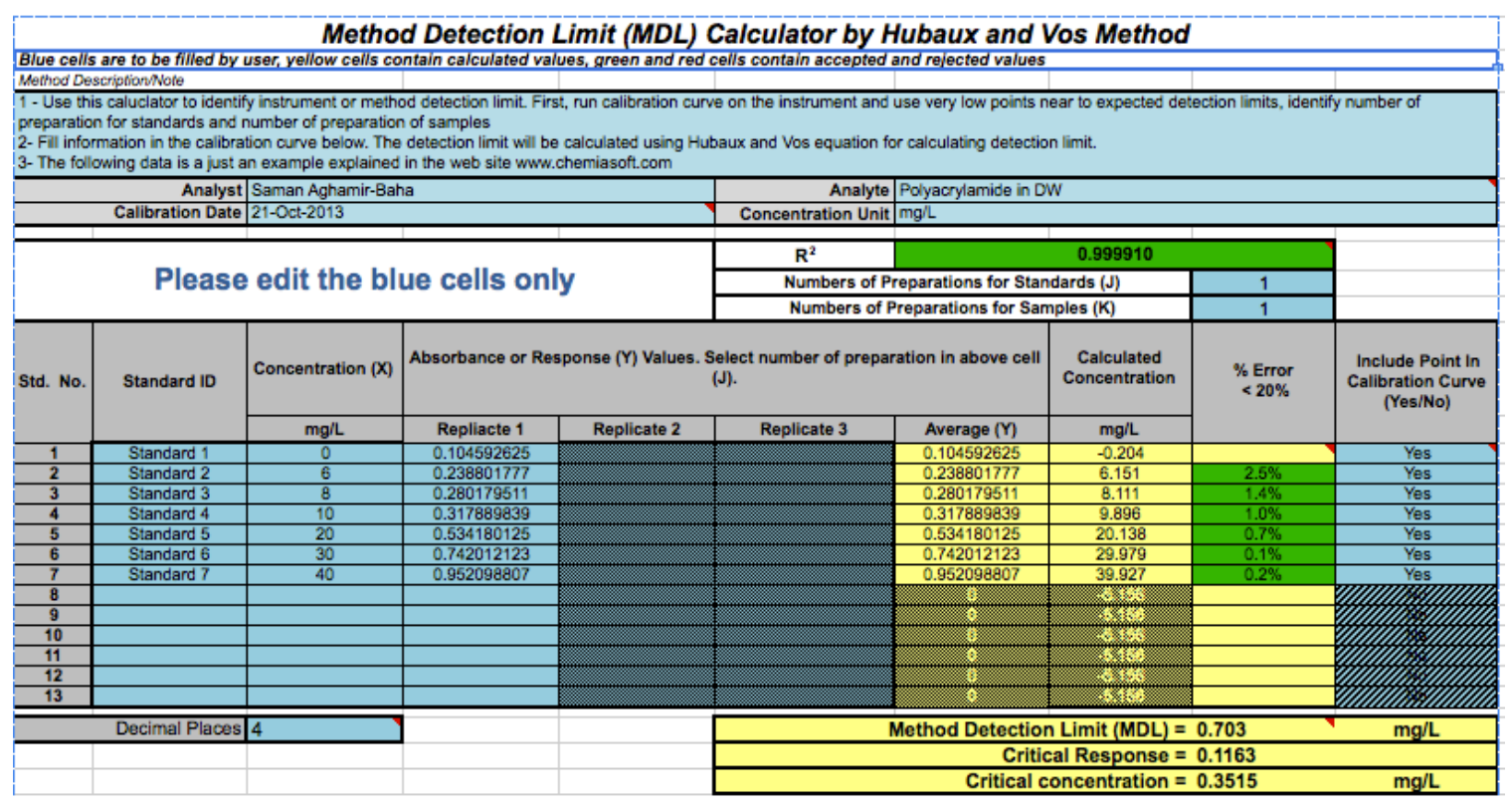

Figure A.1 A snap shot of the automated excel sheet from chemiasoft website (Chemiasoft.com, accessed February 2014) for MDL calculation using SNF475 polymer samples in DW 


\section{Appendix B - Supplementary Figures of Chapter 3}

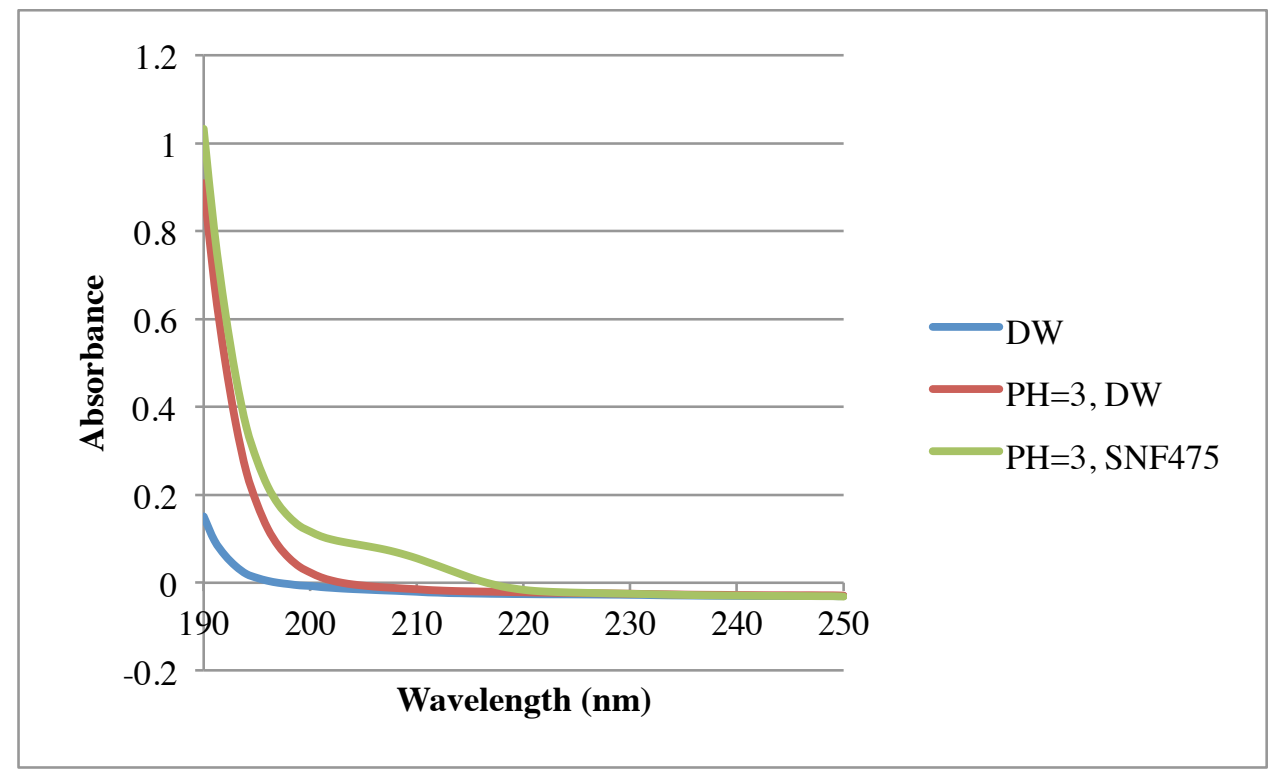

Figure B.1 Effects of $\mathrm{pH}=3$ on DW, and $5 \mathrm{mg} / \mathrm{L}$ solution of SNF475 in DW (average of three measurements for replicate 2)

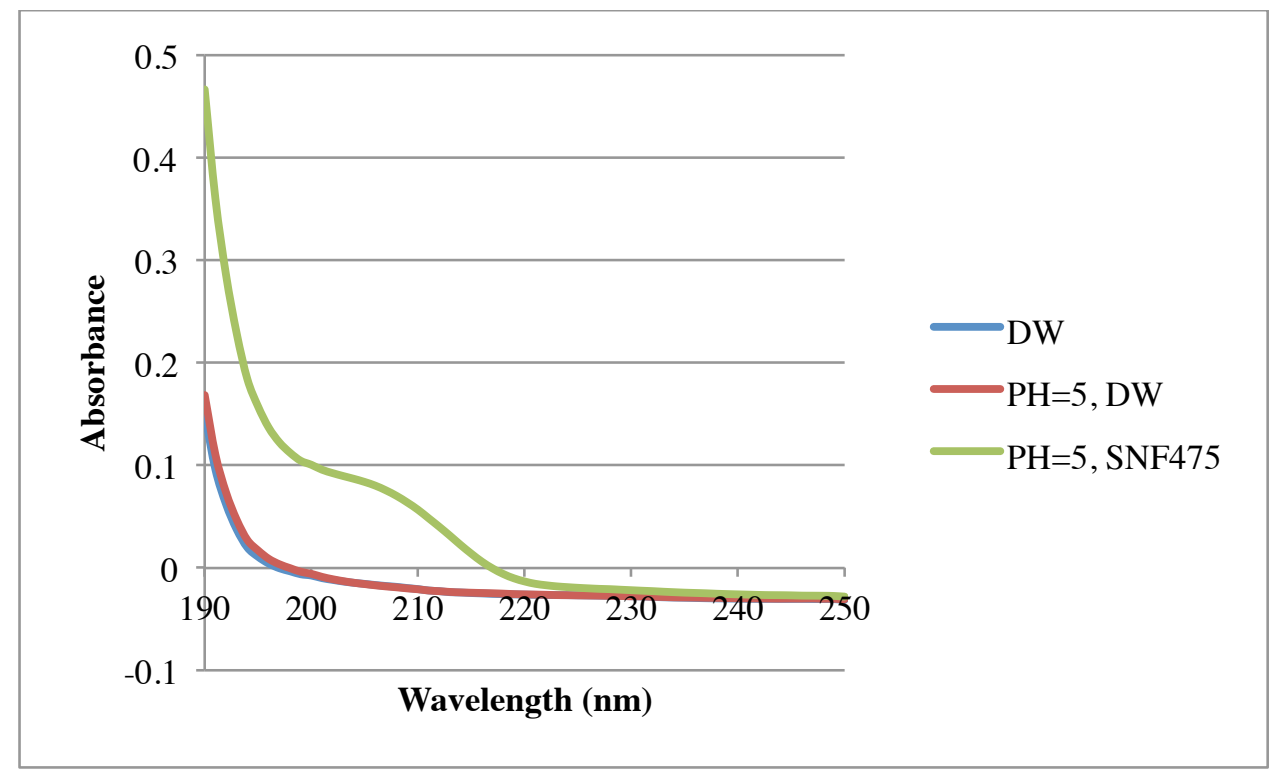

Figure B.2 Effects of $\mathrm{pH}=5 \mathrm{on} \mathrm{DW}$, and $5 \mathrm{mg} / \mathrm{L}$ solution of SNF475 in DW (average of three measurements for replicate 2) 


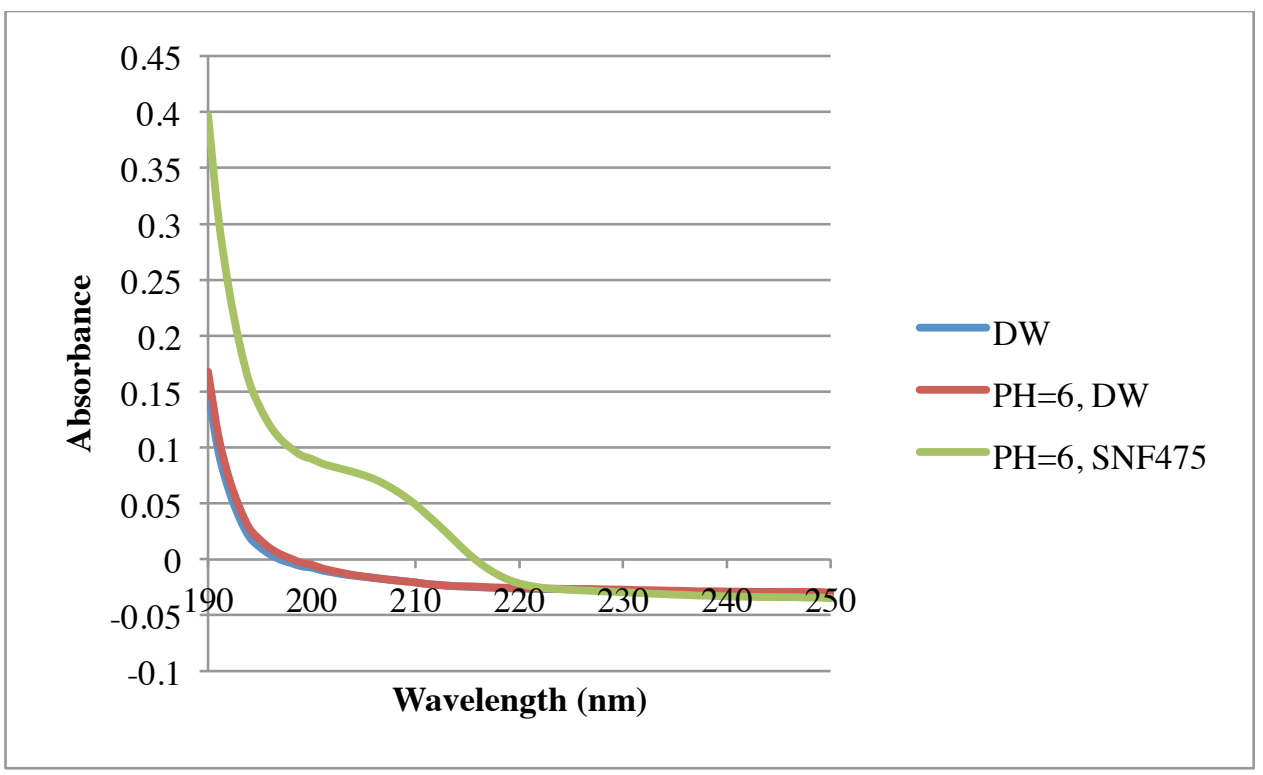

Figure B.3 Effects of $\mathrm{pH}=6$ on DW, and $5 \mathrm{mg} / \mathrm{L}$ solution of SNF475 in DW (average of three measurements for replicate 2)

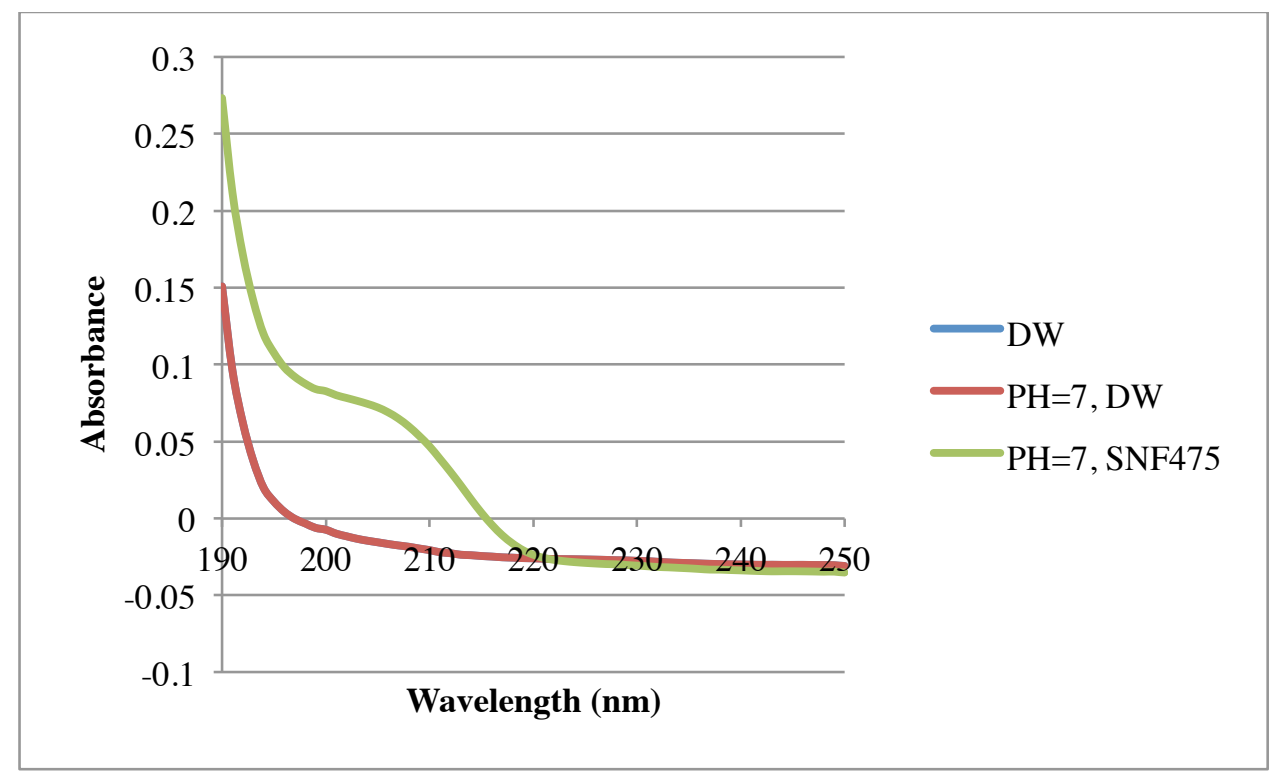

Figure B.4 Effects of $\mathrm{pH}=7$ on DW, and $5 \mathrm{mg} / \mathrm{L}$ solution of SNF475 in DW (average of three measurements for replicate 2) 


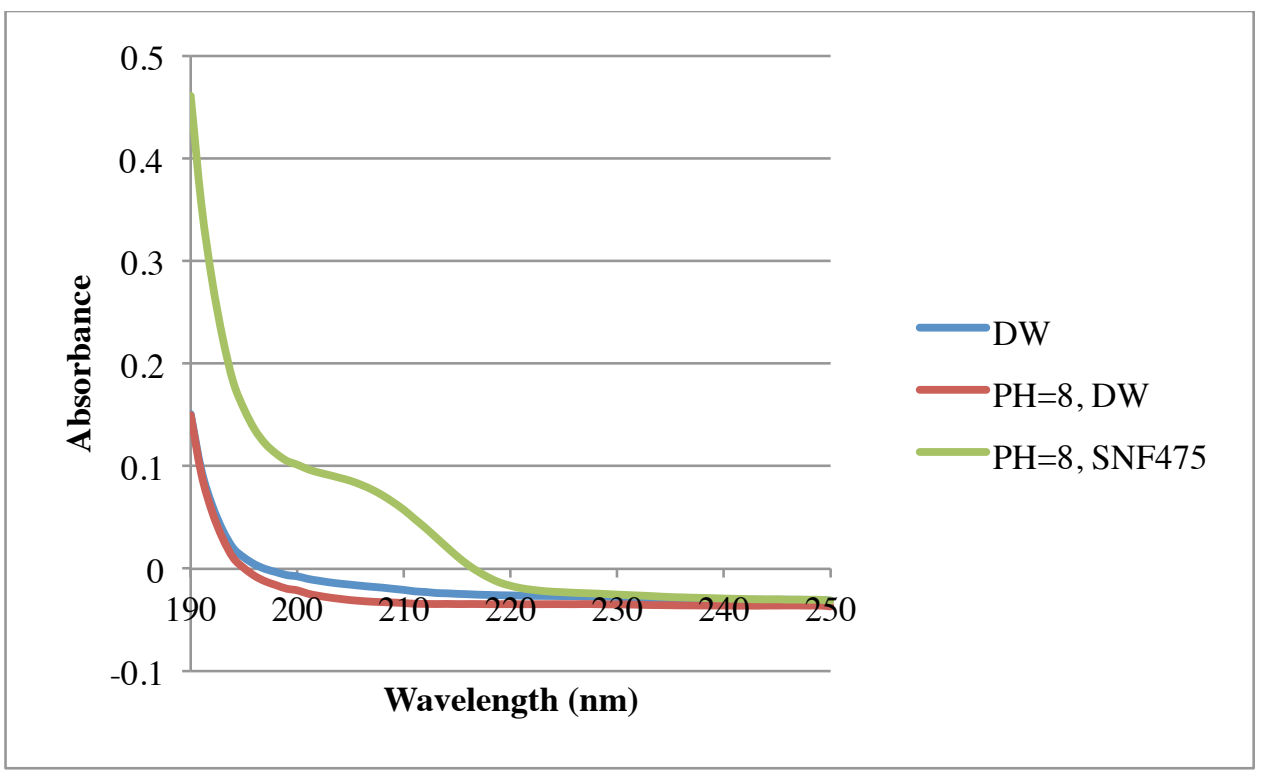

Figure B.5 Effects of $\mathrm{pH}=8$ on DW, and $5 \mathrm{mg} / \mathrm{L}$ solution of SNF475 in DW (average of three measurements for replicate 2)

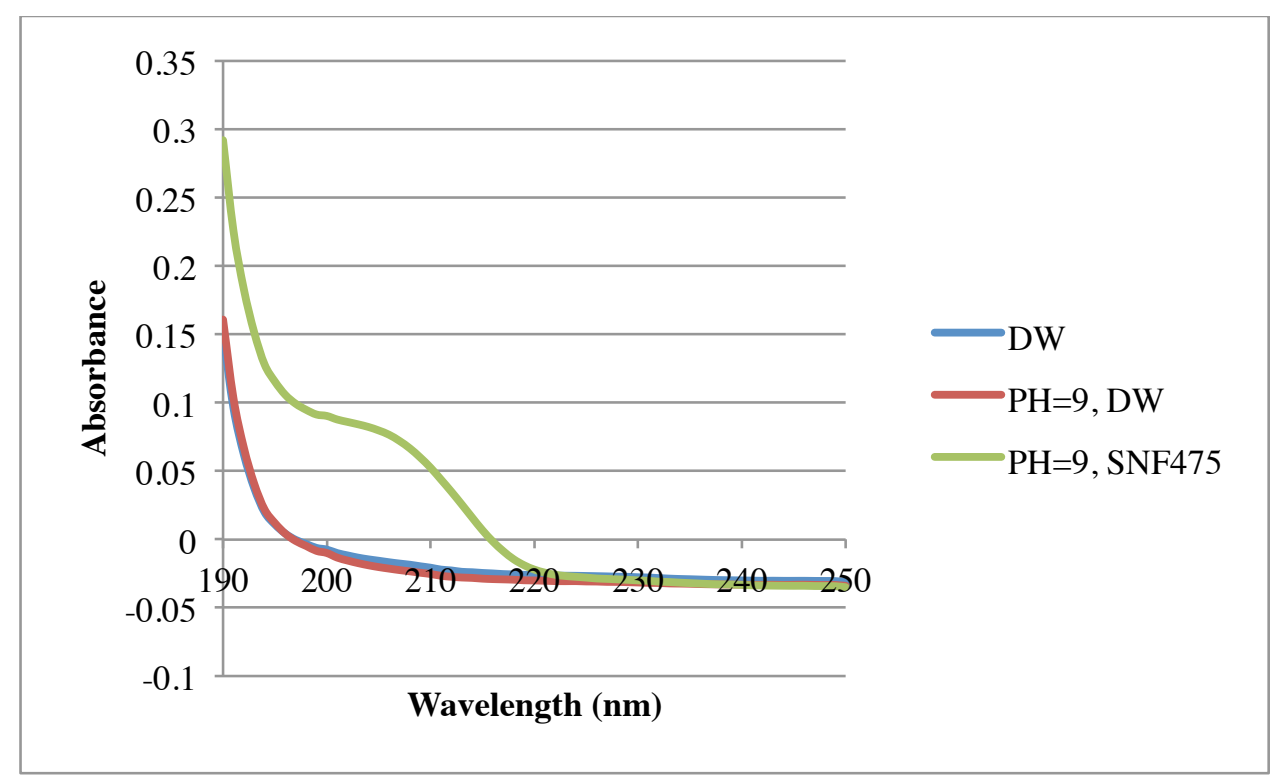

Figure B.6 Effects of $\mathrm{pH}=9$ on DW, and $5 \mathrm{mg} / \mathrm{L}$ solution of SNF475 in DW (average of three measurements for replicate 2) 


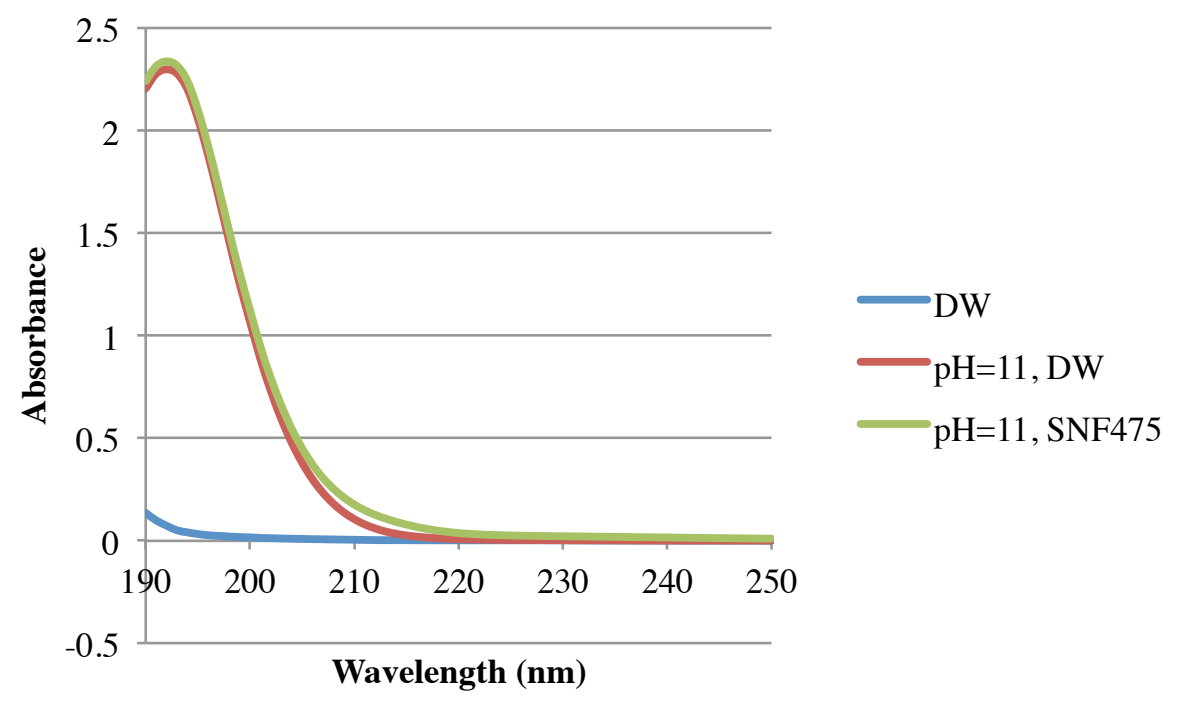

Figure B.7 Effects of $\mathrm{pH}=11 \mathrm{on} \mathrm{DW}$, and $5 \mathrm{mg} / \mathrm{L}$ solution of SNF475 in DW (average of three measurements for replicate 2) 


\section{References}

1. Amuda, O. S., and I. A. Amoo. "Coagulation/flocculation process and sludge conditioning in beverage industrial wastewater treatment." Journal of Hazardous Materials, 2007: 778-783.

2. Arryanto, Y., and L. S. Bark. "Improved and rapid method for the spectrofluorimetric determination of trace amounts of polyacrylamides in waters." Analyst, 1991: 11491153.

3. Benitez, Jaime, Abraham Rodriquez, and Alejandro Suarez. "Optimization technique for sewage sludge conditioning with polymer and skeleton builders." Journal of Polymer Science, 1994: 2067-2073.

4. Budd, Scott S., Narasimha M. Rao, Jitendra Shah, and Ananthasubra Sivakumar. Fluorescent tracer in a water treatment process. USA Patent US5413719 A. 1995.

5. Campbell, Herbert W., and Phillip J. Crescuolo. Process and apparatus for controlled addition of conditioning material to sewage sludge. US Patent US4544489 A. 1985.

6. Campbell, H. W., R. J. Rush, and R. Tew. "Sludge Dewatering Design Manual." Agreement Research Report, Canada, Ontario, 1978.

7. Chemiasoft. "Calculations of MDL by Hubaux and Vos method." Chemiasoft.com http://www.chemiasoft.com/node/60 (accessed February, 2014)

8. Chmilenko, F. A., I. V. Korobova, and S. V. Nazarenko. "Spectrophotometric Determination of Polyacrylamide in Aqueous Solutions Using Cationic Dyes." Analytical Chemistry 59, no. 2 (2004): 124-128. 
9. Chow, C. W. K., J. A. Van Leeuwen, M. Drikas, R. Fabris, K. M. Sparl, and D. W. Page. "The impact of the character of natural organic matter in conventional treatment with alum." Water Science Technology 40, no. 9 (1999): 97-104.

10. Christensen, J. R., P. B. Sorensen, G. L. Christensen, and J. A. Hansen. "Mechanism for Overdosing in Sludge Conditioning." Journal of Environmental Engineering 119, no. 1 (1993): 159-171.

11. Crummett, W. B., and R. A. Hummel. "Determination of Traces of Polyacrylamides in Water." J. Am. Water Wks. Assoc. 55 (1963): 209-219.

12. Daughton, Christian G. "Quantitation of Acrylamide (and Polyacrylamide)." The California Publich Health Foundation, 1988.

13. De Milieux, Zac. Sludge Dewatering Brochure. France: SNF FLOERGER, 2003.

14. Dentel, Steven K. "Evaluation and role of rheological properties in sludge managements." Journal of Water Science Technology, 1997: 662-668.

15. Dentel, Steven K., Mohammad M. Abu-Orf, and Christopher A. Walker. "Optimization of slurry flocculation and dewatering based on electrokinetic and rheological phenomena." Chemical Engineering Journal, 2000: 65-72.

16. Eisenlauer, J., and D. Horn. "Fiber-Optic Sensor Technique for Flloculant Dose Control in Flowing Suspensions." Colloids and Surfaces 14 (1985): 121-134.

17. Francois, Jeanne, and Houchang Kheradmand. "Hydrolysis of Polyacrylamide and Acrylic Acid-Acrylamide Copolymers at Neutral PH and High Temperature." Polymer 29 (1988): 860-870. 
18. Gibbons, Meaghan, and Banu Ormeci. "Quantification of polymer concentration in water using UV-Vis spectroscopy." Journal of Water Supply: Research \& Technology-AQUA 62, no. 4 (2013): 205-213.

19. Hendrickson, E. R., and R. D. Neuman. "Determination of polyacrylamide by spectrofluorometry." Journal of Analytical Chemistry, 1984: 354-358.

20. Howard, G. J., F. Lyth Hudson, and Jeremy West. "Water-Soluble Polymers as Retention Aids in a Model Papermaking System. I. Polyacrylamides." Journal of Applied Polymer Science 21 (1977): 1-16.

21. Hubaux, Andre, and Gilbert Vos. "Decision and Detection Limits for Linear Calibration Curves." Analytical Chemistry 42, no. 8 (1970): 849-855.

22. Korshin, Gregory V., Ch-Wang Li, and Mark M. Benjamin. "Monitoring the properties of natural organic matter through UV spectroscopy: A consistent theory." Water Resources 31, no. 7 (1997): 1787-1795.

23. Lakowicz, Josep R. Principles of Fluorescence Spectroscopy. Baltimore: Springer Science plus Business Media, LLC, 2006.

24. Lambert, Jack L., and Fred Zitomer. "Simplified Preparation of Cadmium IodideLinear Starch Reagent for Colorimetric Iodimetry." Analytical Chemistry, 1963: 405.

25. Lee, C. H., and J. C. Liu. "Enhanced sludge dewatering by dual polyelectrolyte conditioning." Journal of Water Resources, 2000: 4430-4436.

26. Lu, Jianhang, and Laosheng Wu. "Polyacrylamide Quantification Methods in Soil Conservation Studies." Soil and Water Conservation 58.5 (2003): 270-281. 
27. Lu, Jianhang, Laosheng Wu, and Jianying Gan. "Determination of Polyacrylamide in Soil Waters by Size exclusion Chromatography." J. Environ. Qual., no. 32 (2003): 1922-1926.

28. Muller, G., J. P. Laine, and J. C. Fenyo. "High-molecular-weight hydrolyzed polyacrylamides. I. Characterization. Effects of salts on the conformational properties." Journal of Polymer Science; Polymer Chemistry 17 (1979): 659-672.

29. Scoggins, M. W., and J. W. Miller. "Determination of Water-Soluble Polymers Containing Primary Amide Groups Using the Starch-Triiodide Method." Society of Petroleum Engineers of AIME, 1979: 151-154.

30. Scoggins, M. W., and John W. Miller. "Spectrophotometric Determination of Water Soluble Organic Amides." Analytical Chemistry 47, no. 1 (1975): 152-154.

31. Shen, Jun-Ju, Lei-Lei Ren, and Yuan-Yi Zhuang. "Interaction between anionic dyes and cationic flocculant $\mathrm{P}(\mathrm{AM}-\mathrm{DMC})$ in synthetic solutions." Journal of Hazardous Material, 2006: 809-815.

32. Taylor, Kevin C., and Hisham A. Nasr-El-Din. "Acrylamide copolymers: A review of methods for the determination of concentration and degree of hydrolysis." Journal of Pertoleum Schience and Engineering 12 (1994): 9-23.

33. Truong, N. D., J. C. Galin, and J. Francois. "Microstructure of acrylamide-arclyc acid copolymers: 1. As obtained by alkaline hydrolysis." Polymer, 1986: 459-466.

34. Vesilind, P. Aarne. "Capillary Suction Time as a Fundamental Measure of Sludge Dewaterability." Water Pollution Control Federation, 1988: 215-220. 
35. Zuman, Petr, and Wayne Szafranski. "Ultraviolet Spectra of Hydroxide, Alkoxide, and Hydrogen Sulfide Anions." Journal of Analytical Chemistry 48, no. 14 (1976): 2162-2163. 